\title{
PROGRAMMING IN THE META-LANGUAGE: A TUTORIAL
}

Dines Bjorner

\section{Abstㅁaㅡㅌ:}

This paper provides an informal introduction to the "art" of abstractly specifying software architectures using the VDM meta-language*. A formal treatment of the semantics, as well as a BNF-like concrete syntax, of a large subset of the meta-language is given in [Jones 78a] following this paper.

* colloquially known as: META-IV 
CONTENTS

Part I: Prelude 31

0. Introduction 32

Example 0

1. Overview of Meta-Language 43

1.1 Abstract Data Types 43

1.2 Combinators: Statements and Structured Expressions 44

1.3 Abstract Syntax 46

1.4 Logic 46

-- Quantified Dxpressions 47

$\begin{array}{ll}1.5 \text { Descriptor Expressions } & 47\end{array}$

1.6 Undefined \& Erroneous Situations 47

1.7 User-Defined Identifiers 48

1.8 Structure of Tutorial 50

Part II: Domains, Objects \& Operations 51

2. SETS 52

Example I 52

2.1 Defining Domains of SET Objects 54

Example 2-3 54

2.2 Representing Instances of SET objects 55

2.2.1 Explicit Enumeration 55

The Empty Set $\quad 55$

2.2.2 Implicit Enumeration $\quad 55$

Examples $4-5-6 \quad 56$

2.3 SET Operations 56

Example $7 \quad 58$

2.4 SET-oriented Combinators 59

Example $8 \quad 60$

2.5 Further Examples $(9,10) \quad 61,63$ 
3. TUPLES 65

Example 1I 65

3.1 Defining Domains of TUPLE Objects 67

Example $12 \quad 67$

3.2 Representing Instances of TUPLE Objects 68

3.2.1 Explicit Enumeration 68

The Empty Tuple 69

3.2.2 Implicit Enumeration -- Pt.1 69

Example $13 \quad 69$

3.2 .3 Element ordering $\quad 70$

3.2.4 Implicit Enumeration -- Pt.2 71

Examples $14-15 \quad 72$

3.3 TUPLE Operations $\quad 73$

Examples 16-17 74

3.4 TUPLE-oriented Combinators 75

Example $18 \quad 76$

4. MAPs 77

Example $19 \quad 77$

4.1 Defining Domains of MAP Objects 81

Example $20 \quad 81$

Defining MAP Domains -- Cont'd. 82

Example $21 \quad 12$

Defining MAP Domains -- Cont'd. 82

Example $22 \quad 83$

Defining MAP Domains -- Term'd. 83

Example $23 \quad 83$

4.2 Representing Instances of MAP Objects 84

4.2.1 Explicit Enumeration 84

The Empty Map $\quad 85$

Example $24 \quad 85$

4.2.2 Implicit Enumeration 86

Example $25 \quad 87$

4.2 .3 A Note on Scopes $\quad 88$

Example $26 \quad 88$

4.3 MAP Operations 90

Example $27 \quad 91$

4.4 MAP-oriented Combinators 93

Example 28 
4.5 Further Examples 93

Introductory Example Cont'd -- 29

Another Example -- $30 \quad 96$

A Last Exampie -- $31 \quad 99$

5. TREES 102

Examples $32-33 \quad 102$

5.1 Defining Domains of TREE Objects 104

Example $34 \quad 105$

5.1.1 Tree Constructor Axioms 105

Examples 35-36 106

5.2 Representing Instances of TREE Objects 108

5.3 TREE Operations: Selector Functions 109

Explicitly Defined Selector Function Names 109

Implicitly Defined Selector Function Names 110

$\begin{array}{ll}\text { Example } 37 & 110\end{array}$

Non-Unique selector Function Name Convention 110

Example $38 \quad 111$

6. FUNCTIONS 113

Examples 39-40 114

6.1 Defining Domains of FunCTion Objects 115

Example $41 \quad 115$

6.2 Representing Instances of FunCTion objects 116

$\begin{array}{ll}\text { Functional Abstraction } & 117\end{array}$

$\lambda$-Expressions 118

Free- \& Bound Variables -- Scope 121

Application 122

Definition vs. Application 123

The $Y$ Operator 123

6.3 FunCTion Operations 125

Example $42 \quad 125$

7. ABSTRACT SYNTAX 128

$\begin{array}{ll}\text { Example } 43 & 128\end{array}$

7.1 Domains of Abstract Objects 129

Syntactic \& Semantic Domains 130

Domain Definitions \& Compositions 131

Definitions/Rules 132

Compositions/Domain Expressions 132

Domain Operators 132

The Scope Delimiting (...) Operator 134

Infix Operator Commutativity \& Associativity 134

Examples 44-45-46 134

Semantics of the / Operator 135

Constructing Disjoint Domains 136 
7.2 Abstract Syntaxes Rules 137

Context Constraint $\quad 137,139$

is-Function $\quad 137$

Example $47 \quad 138$

Semantics of Abstract Syntaxes $\quad 139$

Example $48 \quad 140$

7.3 Abstract Syntax-oriented Combinators 141

The McCarthy Conditional Clause 141

The Cases Conditional Clause 141

Example $49 \quad 141$

$\begin{array}{ll}\text { Part III Combinators } & 144\end{array}$

8. Variables 145

Example $50 \quad 145$

8.1 Declarations \& The State 146

$\begin{array}{ll}\text { Example } 51 & 147\end{array}$

8.2 Variable References 148

Prelude $\quad 148$

8.3 Assignment $\quad 149$

Example $52 \quad 149$

8.4 Derived References 152

Sub-References to TUPLE Elements 153

Sub-References to MAP Range Elements 153

Sub-References to Sub-"TREE"s 154

Discussion $\quad 154$

9. Structured Clauses 155

9.1 Overview 155

Conditional Clauses $\quad 155$

Iterative Clauses $\quad 156$

Examples - $53 \quad 156$

9.2 Detailed Syntax \& Semantics 157

9.2.1 If-then-else Conditional $\quad 157$

Schema $\quad 157$

Meaning $\quad 157$

Programing Notes $\quad 157$

9.2.2 McCarthy Conditional 158

Schema 158

Meaning 159

Programming Note $\quad 159$ 
9.2.3 The Cases Conditional 160

Schema 160

Programing Note 160

Meaning 161

Name Binding \& Scope 161

Example $54 \quad 163$

Abstract Syntax 163

Semantic Functions $\quad 164$

9.2.4 The ordered, Iterative For-To-Do Statement 165

Schema 165

Programming Note 165

The Controlled Variable 165

Name Binding \& Scope 165

Meaning 165

Example $55 \quad 166$

Further Examples 56-57 168

9.2.5 The Unordered, For-AZZ \& Parallel statements 169

The For-AlZ Statement 169

Schema 169

The Controlled Variable 169

Name Binding \& Scope 169

Meaning 170

The Parallel Statement $\quad 170$

Schema 170

Meaning 170

Examples 58-59 170

9.2.6 The While-Do statement 171

Schema $\quad 171$

Programming Note $\quad 172$

Meaning $\quad 172$

Examples: $\quad 172-173$

Example $60 \quad 172$

Example $61 \quad 173$

10. Blocks 174

Reference to Block Examples 174

The Block Concept 175

10.1 let Constructs 176

The Syntactic let Construct 176

The Semantic let Construct $\quad 176$

Let Construct variants $\quad 176$

Composite Object let Decompositions 177

Simultaneous \& Recursive let Definitions 178

$\begin{array}{ll}\text { Notational Conventions } & 179\end{array}$ 
10.2 Pure \& Impure Expressions 180

10.3 The " $; "$ Combinator 181

10.4 Compound Statements \& Statement Sequences 182

10.5 Statement- \& Expression Blocks 182

$\begin{array}{ll}10.6 \text { Return } & 183\end{array}$

11. EXITS 184

Example $62 \quad 184$

11.I The EXIT Mechanisms 187

11.2 Pragmatics \& Semantics of the EXIT Mechanism 189

Scope Rules 190

Some Equivalence Transformations 191

11.3 Examples: No. $63 \quad 192$

No. $64 \quad 194$

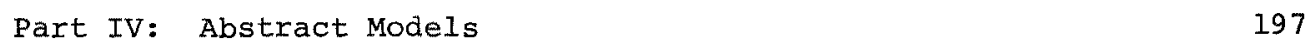

12. Function Definitions \& Abstract Mode1s 198

Example $\quad 198$

12.1 The Syntax of Function Definitions 198

Example $65 \quad 199$

Example $66 \quad 200$

Schönfinckeling/Currying 201

Formal Examples 201

Formal Parameter Syntactic Transformations 202

12.2 The Semantics of Function Definitions

- \& Abstract Models 203

Scope \& Binding 203

General. 203

Examples 203

A Note on Input/Output $\quad 204$

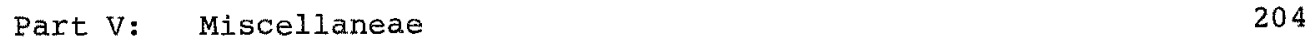

13. ELEMentary Data Types 205

13.I Rational Numbers 206

Programming Note: Numbers \& Numerals 207

13.2 BOOLeans \& Logic Expressions 207

Predicate Expressions 207

Examples 208

$\begin{array}{ll}\text { Comments } & 208\end{array}$ 
13.3 QUOTations

13.4 TOKENS

Example 68

Part VI: Postlude

Annotated Index to Examples

$213-217$

PART I: PRELUDE

Section 0 frames the subject, and gives an extensively annotated exam= ple. This example illustrates many of the important aspects of the me= ta-language. The particular abstraction choices made are, however, not commented upon, nor explicitly singled out.

Section 1 forms an overview of the various meta-language constructs. Although this primer is almost 200 pages (long), the meta-language, as it transpires from section 1, is not 'big'! Certain meta-language no= tions are considered so common, or simple, that they are not treated beyond section 1. We think here, in particular on sections $1.5-1.7$. 


\section{‥ INTRODUCTION}

This tutorial teaches you the meta-language. The primary aim is to render you fluent in the notation and its meaning, both as a reader and as a writer. That is: both as a user of software architectures abstractIy specified by other people, and as a producer of such documents oneself. The secondary aim is to make you use the meta-language as per its intentions, i.e. in good style. We wish that you be able to produce suitable software abstractions. Thus we emphasize giving a rather complete, yet informal coverage of the syntax \& semantics of the meta-language. We refrain however, from presenting a comprehensive introduction to the kind of abstraction principles and techniques which the metalanguage is especially designed to facilitate and express. The tutorial contains an extensive set of examples. A careful study of these is intended to give you a rather complete overview of the fundamentals of abstract modeling -- as we see this activity. See also [Bjфrner 78c].

The meta-language, as already mentioned in the introduction to this volume, evolved in the course of documenting a readable, denotational semantics of a PL/I subset [Bekic 74]. It has subsequently, in addition to denotational semantics definitions of other languages, been applied to similarly abstracted, formal definitions of relational data base systems [Hansa] 76, Nilsson 76], components of operating systems and their command \& (job) control languages [Bjprner 780, Madsen 77], as well as more applications oriented software. These all exemplify, or imply, large complexes of software, with many, and intricate facilities. Individual examples of this tutorial, will, however, also illustrate applications to basic algorithmic functions.

The examples of this tutorial can be grouped according to either of two criteria: free-standing examples (illustrating an isolated software concept); versus examples strung together over several sections (usually illustrating systems software). The latter can (furthermore) be grouped according to the kind of systems software being abstracted. The following is a reference guide to these: *

Programing Language Constructs:

Examples: $30-34,36-38,40,42-43,47,49-62,64-68$.

(Most of these examples are mere transcriptions and annotations of parts of the mini-language definition of appendix III [Jones 78a].)

* See pages 213-217 for a complete, annotated index to all examples. 


\section{File System Concepts}

Examples: $3-8,12-13,17-18,20-25,27-28,35$. (Operating or File System) Catalogues/Directories

Examples: $19,29,63$.

Usually major sections will start with a large example illustrating all the main notions introduced by the section. The examples interspersed in the text outlining the individual language constructs are usually far simpler than the more encompassing introductory example. Finally, many sections are trailed by yet additional, larger examples. If you find difficulties in comprehending the introductory examples you may wish to skip these intially. If you find difficulty in understanding the interspersed examples this tutorial will have failed:

(We mention, in passing, that no examples will be given of concurrent system architectures: the subject meta-language was not designed to cater for this [sadiy neglected] area.) Finally some examples will be of a rather formal, or schematized nature; not illustrating 'practical' notions, but paraphrasing principal, or 'theoretical', aspects of the meta-language.

We stress here, as was stressed in the introduction to this volume, that the meta-language is to be used, not for solving algorithmic problems (on a computer), but for specifying, in an implementation-independent way, the architecture (or models) of software. Instead of using informmal English mixed with technical jargon, we offer you a very-high-level 'programming' language. We do not offer an interpreter or compiler for this meta-language. And we have absolutely no intention of ever wasting our time trying to mechanize this meta-language. We wish, as we have done in the past, and as we intend to continue doing in the future, to further develop the notation and to express notions in ways for which no mechanical interpreter system can ever be provided. Given a terse and readable abstract model of some software item, and as e.g. expressed in this meta-language, we see it as the foremost and almost solely distinguishing task of programming to carefully turn the abstraction, in stages of, at most semi-automated, development, into an efficient realization. Once such an implementation has been reached we let the entire, possibly annotated, documentation: the abstract model, as well as all intermediate development stages, serve as the only documentation of the realized software.

To give you a flavor of the meta-language, but not really the kind of software we primarily intend it aimed at, we now give you a rather comprehensive example. 


\section{Example 0}

We will present this example completely formally: first the abstract model, then the annotations. Other than these annotations, which represent a mere reading of the formulae, we do not here explain the formulae. Thus the example is bxought to give you, from the very start, a capsule view of core aspects of the meta-language; as well as an idea about what a model is: its parts, their purpose and interrelations.

\section{Semantic Domains}

$\begin{array}{lll}1 & \text { GROCER } & \therefore \text { SHELVES STORE CASH CATALOGUE } \\ 2 & \text { SHELVES } & =\text { Wno } \vec{m} N_{1} \\ 3 & \text { STORE } & =\text { Wno } \vec{m} N_{1} \\ 4 & \text { CASH } & =N_{0} \\ 5 & \text { CATALOGUE } & =\text { Wno } \vec{m} \text { Description } \\ 6 & \text { Description } & \therefore \text { Price Minimum Maximum Size } \\ 7 & \text { Price } & =N_{1} \\ 8 & \text { Minimum } & =N_{1} \\ 9 & \text { Maximum } & =N_{1} \\ 10 & \text { Size } & =N_{1}\end{array}$

\section{-- annotations:}

The model is concerned with rather self-contained fragments of a grocery: its inventory, cash and catalogue subsystem. The model describes a domain of such groceries and exemplifies a few of the manipulations that groceries are subject to: customer purchases, and inventory/cash control.

The reader is, throughout this primer, well-adviced in reading the annotations with a finger following the formulae and trying, otherwise, to establish the connection:

1 A grocery is here selectively abstracted by abstractions of its shelves and store, i.e. inventory, its cash register, and its catalogue.

2 The shelves display a finite, non-zero number of items of a finite variety of merchandise. Merchandise presently being abstracted by ware number codes. 
In the store-room is similarly kept a finite, non-zero number of boxed quantities of items of a finite selection of wares. The cash register is simply abstracted by the cash it contains. The grocers' catalogue lists a description of each sort of merchandise.

Such a description here consists of the unit (item) sales price; the minimum and maximum (lower- \& upper-bound) numbers of items, of the described merchandise, which ought, respectively may be placed on the shelves; and finally the size of a stored box, measured in terms of the number of merchandise items it contains. Prices are measured in integer (positive number) units of currency.

etc. .

\section{Comments}

The above description 'read' the formulae as describing a grocery. The formulae, in fact, defines a whole domain (i.e. class) of such.

The formulae (1-10), and (12-15) below, constitute an abstract syntax (abstract syntaxes). Each line $(1,2, \ldots, 10)$ is an abstract syntax rule. The rules all have their left-hand sides being identifiers. The right-hand sides are so-called domain-expressions.

The described domains are said to constitute the semantic domains. Semantic domains are "what the whole thing is about". Syntactic domains, described below in formulae 12-14, are (just the) objects which denote manipulations of semantic objects. 


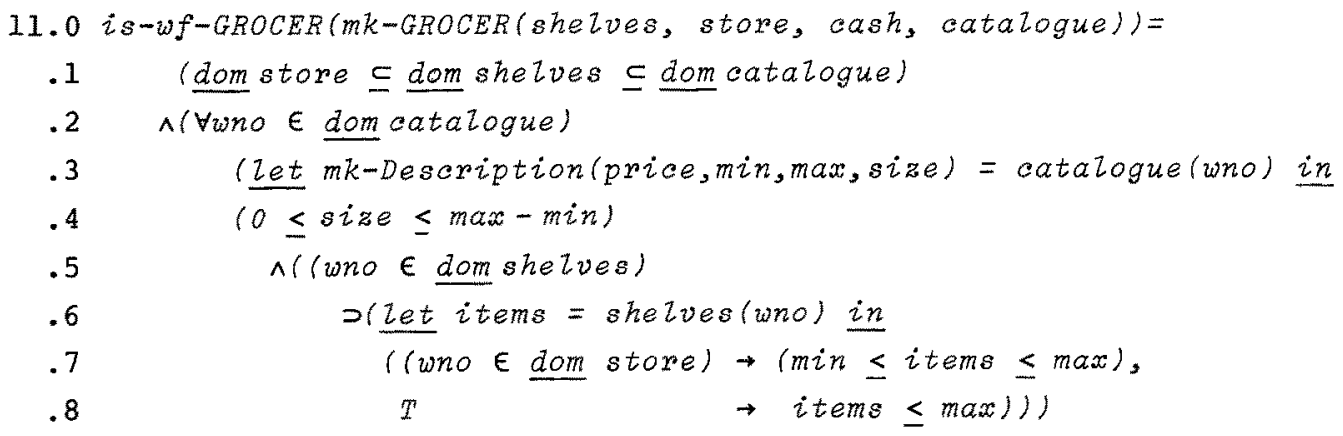

\section{- annotatitions :}

The domain descriptions captured the essence of how we abstractly view groceries, but the defined domains contain objects, i.e. groceries, which do not satisfy natural constraints:

11.0 For a grocery (which consists of shelves, a store room, the cash register, and a catalogue) to be well-formed, the following constraints must be satisfied:

11.1 There cannot be merchandise in the store room which is not also displayed on the shelves; and any merchandise on the shelves must be described in the catalogue. Furthermore:

11.2 For each type of merchandise described in the catalogue,

11.3 look-up the price, minimum \& maximum shelf-, and box size quantities for that ware in the catalogue:

11.4 Now the maximum must be higher than or equal to the minimum, and their difference (in that order) must be lower than or equal to the box size. (This is a pragmatic constraint. It permits the update of shelves with the full contents of boxes, without violating (min, max) constraints.)

11.5 If, in addition, the ware is also, actually displayed on a shelf, then:

11.6 Let us call the number of items of that ware on the shelves for items. Now:

11. If the ware additionally is further stored in the back room, then the number of items on the shelves must actually fall between the 
minimum, lower and maximum, upper bounds; otherwise

11.8 there can be no more items on the shelves than is maximally permitted.

\section{Comment:}

The is-wf-function is defined relative to some named domain, here the class of all GROCERies. In the following we shall understand by GROCER the class of those objects which satisfy the predicate (11). In fact, any manipulation, i.e. any transformation of, or process on, a grocery, shall leave us a(nother) grocery also satisfying the well-formedness constraint. Thus the predicate (11) is also seen as forming a major part of the invariant according to which we 'program' our manipulations -- whether on the abstract, or concrete level.

\section{Syntactic Domains}

12 Transaction = Purchase $\mid$ Control $\mid \ldots$

13 Purchase :: Wnot

14 Control $\because$ Wno-set

$15 \quad \cdots$

\section{- annotatations:}

The grocer sees a customer purchase, his own daily check on the availability of certain wares, etc., as transactions. These are operations on the grocery. Syntacticaliy speaking:

12 A transaction is either a customer purchase, a clerk control, or something else - presently undefined:

13 A customer purchase is presented at the check-out counter as a sequence of not necessarily distinct wares. In this sequence groups of items of the same kind need not occur together.

14 A store clerk control consists of a set of distinct ware types (for which their inventory amounts are asked). 


\section{Comment:}

Observe that only the last parenthesized phrase above invoked semantic notions. That is: we have totally separated syntactic descriptions from those of their semantics.

\section{Dynamic Consistency Constraints}

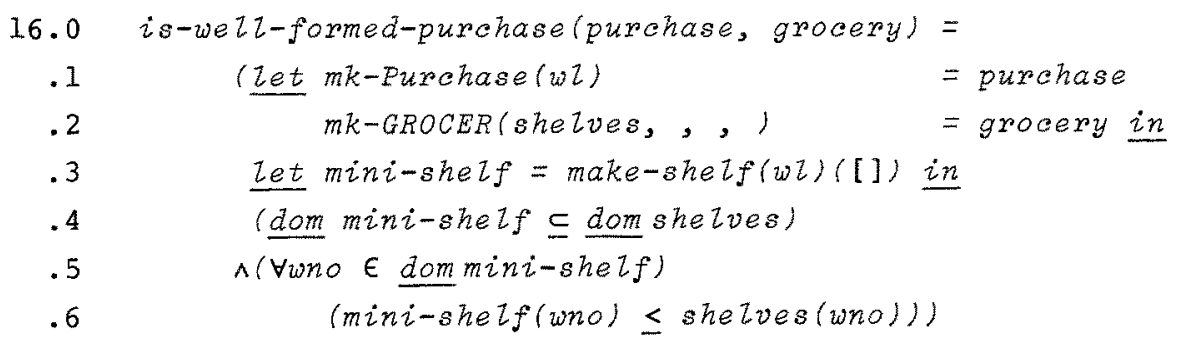

\section{- annotatations :}

The customer can select merchandise only from the shelves, and in quantities bounded by what is displayed:

16.0 For the combination: a purchase and a grocery to be consistent, we must therefore require that

16.1 the ware list of which the purchase is made up, and

16.2 the shelves (from which the wares were selected) satisfy the constraints given in lines 16.4-16.6.

16.3 To express those constraints let us compute for each ware type, in the purchase, the number of times it occurs in the purchase. since this corresponds, in the way we abstracted shelves, to an object of SHELVES, we call it mini-shelf. That is: mini-she if displays, as do shelves, a functional association between ware codes and numbers of (shelves, mini-shelf) items of that ware. The function make-shelf, which takes the ware-list of the purchase and computes this association, is activated with an empty shelf. The function definition is given below (17). Now to the constraints themselves:

16.4 The kind of wares purchased must form a (not necessarily proper) subset of the merchandise displayed on the shelves. 
And :

16.5 For each ware purchased

16.6 the number of items purchased must be less than or equal to the number of wares of that kind on the shelves.

\section{Comment}

The abstraction given is not as elegant and transparent as we would have hoped. Thus we find the introduction of the make-sherf auxiliary function somewhat of a resort to an operational description of the constraint.

\section{Auxiliary Function}

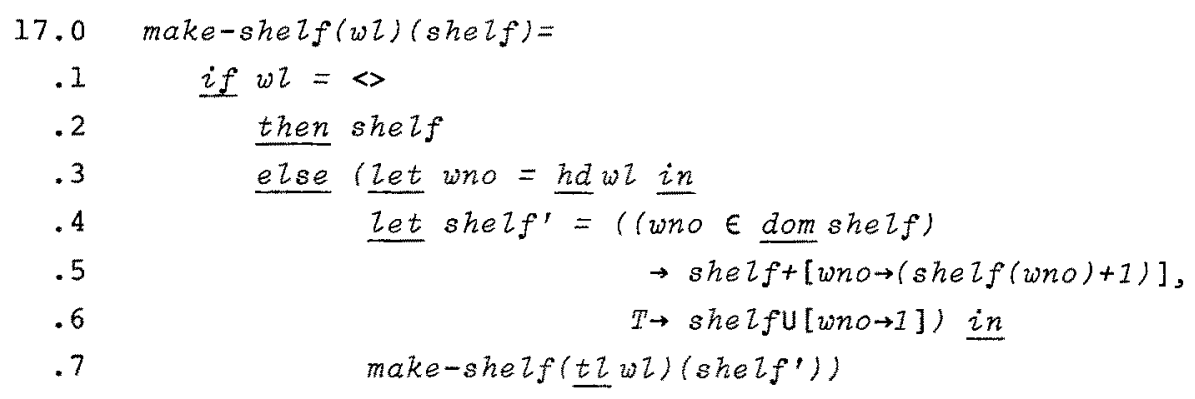

type: Wno $^{+} \rightarrow$ (SHELVES $\rightarrow$ SHELVES)

\section{- annotations :}

The function takes, as arguments, a tuple (or list) of wares and a shelf (really: an object of SHELVES) and yields, as result, a SHELVES object. The type clause expresses this. The form after the type colon is a so-called domain expression. The function is recursively defined, cf. Iine 17.7. It performs its function by treating each item (wno) in the ware list separately: 17.3-17.6. That is: by 'chopping' off the list from its front, or head, leaving treatment of the rest, i.e. $\underline{t} a i \underline{l}$, of the list, till another recursion (17.7), passing it the shelves object (shetf') so far computed. Thus:

17.I If the (tail of the) ware list is the null tuple,

17.2 then the accumulated shelf computed up till now is delivered as the result. 
17.3 Otherwise: Let us call the first item of the remaining ware list for wno.

17.4 And then update the shelf so far computed. If the item under inspection (being "tallied") has already occurred in the ware list,

17.5 then the updated shelf has one added to the number of items, of the sarne kind, so far purchased.

17.6 Otherwise, this is a first occurrence of this particular ware, and the she $f f$ is augmented by the initialization of its tally count to 1 .

\section{Semantic Functions}

18.0 Elab-Purchase(mk-Purchase(wi), grocer $)=$

\section{.1}

.2

.3

.4

.5

.6

.7

.8

.9

.10

.11

.12

.13

.14

.15

.16

.17

.18

.19

.20

.21

if $w z=<$

then grocer

else (Let mk-GROCER (shs, sto, cash,cat) = grocer, $m k-$ Description $(p, \min , \max$, size) $=\operatorname{cat}($ hd $w i)$,

wno

$=\underline{h d} w \tau$

let $c a s h^{\prime}=$ cash+p, (shs', sto') $=$

( Let items $=$ shs $($ wno $)$, stored $=($ (wno $\in$ dom sto $) \rightarrow$ sto (wno $)$,

$T \quad \rightarrow$ o) $\underline{i n}$

if $($ (items $=m i n) \wedge($ stored $>0))$

then

(shs + [wno $\rightarrow$ items-1+size],

(istored=1) $\rightarrow$ sto $\{$ wno\},

$T \rightarrow$ sto $[$ wno $\rightarrow$ stored -1$]))$

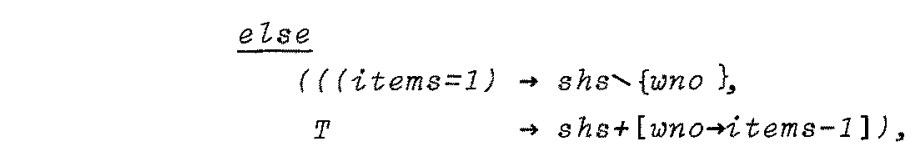

else

sto)) $\underline{i n}$

Elab-Purchase (mk-Purchase(tzw

$m k-G R O C E R\left(s h s^{\prime}\right.$, sto', cash', cat)))

type: Purchase GROCER $\cong$ GROCER

\section{-- annotatations:}

To purchase a ware is to take a grocery store and deliver another. In 
the resulting grocery store the shelf count for the purchased ware (wno) is diminished by $I(18.13 \& 18.18)$, and if the shelf display goes under minimum and thereare supplies stored $(18.11)$ then the shelf is replenished by size items (18.13). To the cash register is added the purchase price of the ware $(18.6)$.

The Purchase Elaboration, as did the above auxiliary function, works by updating the grocery store, once completely for each item in the purchase (18.3-18.19). That is:

18.1 If the purchase has been completely serviced,

18.2 then the result is the "input' grocery.

18.17 If the shelf display of the purchased ware would go to zero, cognizance of this ware is deleted from the shelf. Likewise:

18.14 If by replenishing the shelves from the store the supplies go to zero, this ware is deleted from the store room.

18.20 The rest of the purchase is elaborated.

18.21 with the updated grocery.

19.0 EZab-Control (mk-Controz (wno), grocer $)=$ Tabulate (wno, grocer)([])

type: Control GROCER $\rightarrow\left(\right.$ wno $\left.\vec{m} N_{0}\right)$

\section{-- annotatítions :}

To control the grocery for the quantities available of each of a given set of ware categories is to tabulate these, starting with an empty table.

The tabulate function is auxiliary. The resulting table associates to each ware category the possibly zero quantity on hand, on the shelves as well as in the supply. 


\section{Auxiliary Function}

20.0 Tabulate (wnos, grocer $)($ table $)=$

.1 if wnos=\{\}

.2 then table

.3 else (Let $m k-G R O C E R$ (shs, sto, cat) = grocer,

.4 wno $E$ wnos in

.5

.6

.7

.8

.9

Let items $=(($ wno $\epsilon$ domshs $) \rightarrow$ shs $($ wno $)$,

8

.10

$T \rightarrow 0)$,

stored $=($ (wno $\in$ dom sto $) \rightarrow$ sto $($ wno $)$,

$T \rightarrow 0$ ),

size $=s-S i z e(c a t(w n o))$ in

.11

Let sum = items + (stoxed*size) in

Tabulate (wnos $\{$ wno,$g r o c e r)(t a b$ leu [wno $\rightarrow$ sum $]$

type: Wno-set GROCER $\rightarrow\left(\right.$ (Wno $\left.\vec{m} N_{0}\right) \rightarrow\left(\right.$ Wno $\left.\left.\vec{m} N_{0}\right)\right)$

\section{-- annooations :}

are left to the reader! 


\section{OVERVIEW OF META-LANGUAGE}

In this section we briefly survey the meta-language. The overview of sections 1.1-1.3 emphasizes only syntactic aspects. The aim of sections 1.1-1.3 is to give you a rather comprehensive feeling for the composition and extent of the meta-language.

\section{I Abstract Data Types}

The elementary data types (except for booleans) of the meta-language are not fixed. For a suggestion of suitable base types we refer to section 13 .

The composite, very-high level abstraction-facilitating, data types are:

\section{Sets:}

$$
\begin{array}{ll}
\text { Domains: } & A-s e t \\
\text { Constructors } & \{\ldots\} \\
\text { Operations } & U, \cap,-, \subseteq, \subset, \epsilon, \text { card, power, }=, \neq
\end{array}
$$

\section{Tuples:}

$$
\begin{array}{ll}
\text { Domains: } & A^{*}, A^{+} \\
\text {Constructors: } & <\ldots .> \\
\text { Operations: } & h d, \text { ti, len, ind, elems, },[.], \text { eone, }=, \neq
\end{array}
$$

\section{Maps:}

$$
\begin{array}{ll}
\text { Domains: } & A \vec{m}^{B} \\
\text { Constructors: } & {[\ldots, \ldots]} \\
\text { Operations: } & \underline{\text { dom, }} \text { rng, }, .,, U,+,,,=, \neq
\end{array}
$$

\section{Functions:}

$\begin{array}{ll}\text { Domains : } & A \rightarrow B, A \sim B \\ \text { Constructors : } & \lambda \ldots \bullet . . \\ \text { Operations: } & (.)\end{array}$




\section{Trees:}

Domains:

Constructors:

operations:

$$
\begin{aligned}
& A \quad \therefore \quad B_{1} B_{2} \ldots B_{n}, \quad\left(C_{1} C_{2} \ldots C_{n}\right) \\
& m k-A, m k,(\ldots) \\
& s-B_{i},=, \neq
\end{aligned}
$$

The 'domains' lines above exemplify the expression of domains of objects of the subject type. $A, B, B_{i}$ and $C_{i}$ are given (or defined) domains. The constructors hint at the following typical object construction expressions:

$$
\begin{array}{ll}
\left\{a_{1}, a_{2}, \ldots, a_{n}\right\}, & \{F(d) \mid P(d)\}, \\
<a_{1}, a_{2}, \ldots, a_{n}>, & <F(i) \mid P(i) \wedge m \leq i \leq n> \\
{\left[a_{1} \rightarrow b_{1}, a_{2} \rightarrow b_{2}, \ldots, a_{n} \rightarrow b_{n}\right],} & {[F(0) \rightarrow G(0) \mid P(0)]}
\end{array}
$$

representing respectively explicit \& implicit set, tuple and map object denoting expressions. Also:

$$
\text { גid.clause }
$$

abstracts the meta-language expression or statement clause into the function of id that clause is. Finally:

$$
m k-A\left(b_{1}, b_{2}, \ldots, b_{n}\right), \quad m k\left(c_{1}, c_{2}, \ldots, c_{n}\right) \text { and }\left(c_{1}, a_{2}, \ldots, c_{n}\right)
$$

denote trees.

\subsection{Combinators: Statements and Structured Expressions}

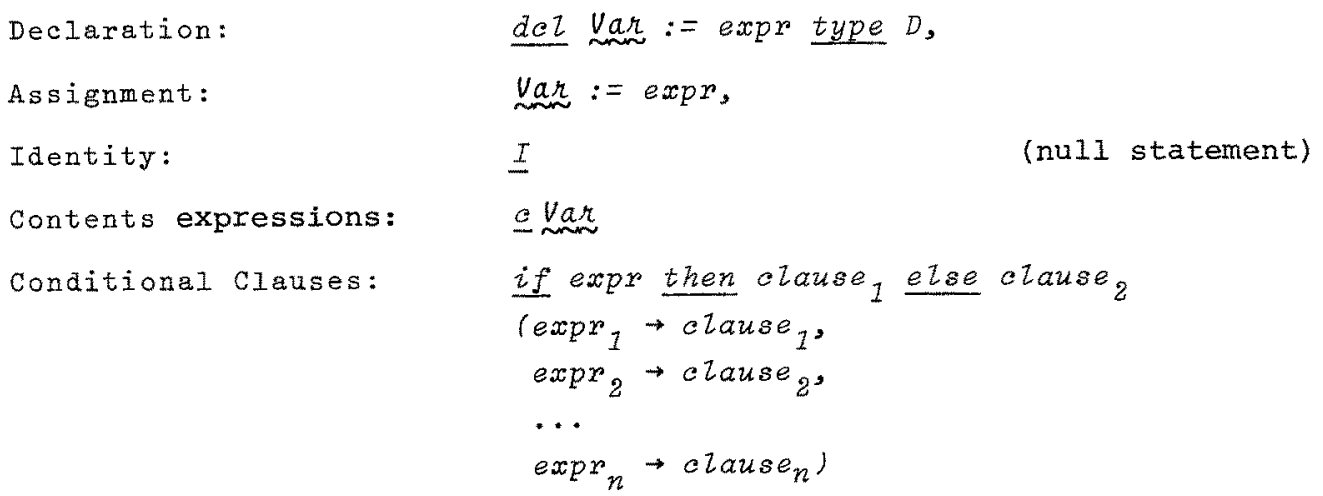

is the classical MeCarthy conditional clause, with: 


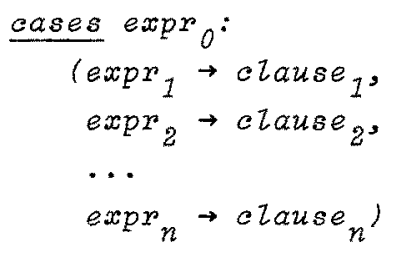

being a variant of the Hoare/wirth cases clause.

whize expr do stmt,

for alz id $\in$ set do stmt, and:

for $i=m$ to $n$ do stmt

are the (conventional) iteration statements. Compound statements use the semicolon operator: (stmt $t_{1}$; stmt $t_{2} ; \ldots$; stmt $t_{n}$ ) -- where stmt are statements. Statement- \& expression blocks may occur anywhere a statement, respectively expression, may occur:

$$
\text { (Zet def }=\text { expr in clause), }
$$

and:

(let def: expr; clause)

illustrate the two block forms: the former basically of the applicative kind, the latter of the imperative kind.

Function definitions are written:

$$
\begin{aligned}
& \text { fid }\left(i d_{1}, i d_{2}, \ldots, i d_{n}\right)= \\
& \text { clause } \\
& \text { type: } D_{1} D_{2} \cdots D_{n} \rightarrow D
\end{aligned}
$$

-- or in some such form. The language has no gotos, but provides both imperative and applicative variants of a phrase-structured, block-oriented, un-labelled exit mechanism;

(trap exit(id) with $F(i d)$ in elause)

and:

$($ tixe $[G(0) \rightarrow F(0) \mid P(0)]$ in clause)

with: 
exit, exit (expr)

being the exit causing constructs.

\subsection{Abstract Syntax}

Abstract syntaxes are used in defining named domains of objects:

$$
\begin{aligned}
& A_{0}=B_{1}\left|B_{2}\right| \ldots \mid B_{n} \\
& A_{1}=B \text {-set } \\
& A_{2}=B^{*} \\
& A_{3}=B^{+} \\
& A_{4}=B_{m} C \\
& A_{5}=B \rightarrow C \\
& A_{6}={ }_{B} \rightarrow C \\
& A_{7} \therefore B_{1} B_{2} \ldots B_{n}
\end{aligned}
$$

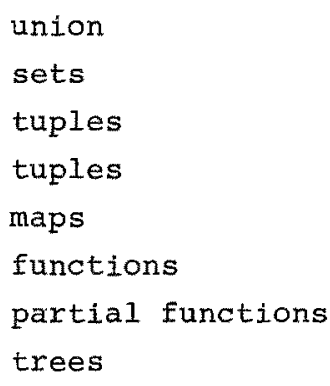

are typical rules. Objects, 0 , in the domains defined by some such rule satisfy:

$$
i s-A_{i}(0) \text {. }
$$

\section{$1.4 \operatorname{LogiC}$}

Only one elementary data type is assumed:

BOOL

consisting of the two truth valued objects:

true, false

to which the following non-commutative operations apply:
$\wedge, v, \supset$
(and, or, implication)

as well as:
$\sim, \equiv$
(negation, identity). 


\section{Quantified Expressions}

Predicates asserting truth properties:

(1) ( $\forall 0 \in$ set) $(P(0))$

(2) $(\exists o \in$ set) $(\mathrm{P}(o))$

(3) ( $\exists ! \circ \in$ set) $(P(o))$

read as follows:

(1) for all objects [in the set] the predicate (P) holds.

(2) there exists an object [in the set] for which the predicate (P) holds.

(3) there exists a unique object [in the set] for which the predicate (P) holds.

\subsection{Descriptor Expression}

This subsection will be the only place in which the descriptor expression is treated.

$$
(10)(P(0)), \quad(\Delta o)(P(0))
$$

1 (iota) and $\Delta$ (delta) are offered as alternate forms of representing the descriptor operator. The forms above denote (and read):

the unique object satisfying the predicate (P).

\subsection{Undefined \& Erroneous Situations}

The form:

\section{undefined}

is offered as a notation expressing some further unspecified undefined object. Applying operations outside their domain is (Iikewise) said to yield undefined results. Similarly for descriptor expressions.

\section{The form:}


is offered as a notation for stating situations for which there are no clear definitions.

In this primer we consistently use undefined to denote undefined (nonstate) objects, i.e. in basically applicative contexts. We consistently use error to denote undefined state transitions, i.e. in basically imperative contexts. Thus we consider undefined to be an expression: error, a statement:

\subsection{User-Defined Identifiers}

The meta-language sets no spelling standard for identifiers naming objects (defined functions, variables, parameters, etc.) or domains (as in abstract syntaxes).

The following outlines the conventions basically followed in this primer.

Identifiers are either single Greek letters or sequences of one or more Roman letters and Arabic digits. Identifiers might contain proper infix hyphens, and possibly be decorated with (simple) subscripts (digits and letters) and/or single, double, triple, ..., (superscript) primes.

The choice between Greek letters and other identifier forms is basically governed by these informal, enumerative rules:

$\begin{array}{ll}\text { (So-called) State Domain Names: } & \Sigma, \text { or } \Xi \\ \text { State object Names: } & \sigma \text {, respectively } \xi \\ \text { (So-called) Environment Domain Names: } & E N V \\ \text { Environment object Names: } & \text { env, or } \rho \\ \text { (So-called) Continuation Domain Names } & C \\ \text { Continuation Object Names: } & \theta\end{array}$

The criterium on when to use upper and lower case letters is basically this:

(So-called) Semantic Domain Names:

Sequences of one or usually more UPPER CASE LETTERS, possibly trailed by a digit. 
(So-called) Syntactic Domain Names:

UPPER case letter followed by one or more lower case letters, possibly trailed by a digit.

Object Names:

Lower case counterparts of corresponding domain names, usually amounting to prefixes of such, and quite often decorated.

Assignable Variable Names:

-- in this primer usually written in script and underlined with tildes. When variable is global, first letter is upper case; otherwise lower-case.

Other conventions are:

Function Names:

Elaboration functions primarily applicable to objects of domain $X y z$ have names:

$\begin{array}{ll}\text { elab-Xyz } & \text { (for imperatively stated) respectively: } \\ E-X y z & \text { for (applicatively expressed) elaboration functions; } \\ \text { int-Xyz } & \text { (for imperatively stated) respectively: } \\ I-X y z & \text { for (applicatively expressed) interpretation func- } \\ & \text { tions; } \\ \text { eval-Xyz } & \text { (for imperatively stated) respectively: } \\ V-X y z & \text { for (applicatively expressed) evaluation functions. }\end{array}$

(Elaboration is a term comprising both interpretation and evaluation. Interpretation implies elaboration of constructs basically for the sake of their side-effects. EValuation implies elaboration of constructs basically for the sake of values explicitly yielded.)

Other Function Names:

Let $A$ be some domain name, then

$$
\text { is }-A, s-A, \quad m k-A, \quad i s-w f-A \text {, }
$$

are reserved names. is-A was explained in section 1.3. $s-A$ and $m k-A$ in 1.1. $i s-w f-A$ is to be the name for any static context 
condition predicate function applicable to all A objects and yielding true only for those $A$ objects one actually intends the definition of $A$ to cover: $s-A$ and $m k-A$ may not actually be defined by some abstract syntax. The above reservation then intends to prevent confusion.

\subsection{Structure of Tutorial}

The structure of this primer is as follows.

In part II we cover data types: sets, tuples, maps, trees, functions and the notion of abstract syntax. Respectively sections $2,3,4,5,6 \& 7$. The language features covered enable us to express domains, objects and primitive operations on these -- in particular operator/operand expressions. Examples of earlier sections will necessarily employ metalanguage notions only formally introduced in later sections. Insofar as this is the case we rely on your good-will and patience, attempting, on our behalf, however, to keep such uses to a reasonable minimum.

In part III we cover language constructs such as variables: their declaration, assignment and contents access; structure expressions and statements; blocks, and the exit mecharism. With these meta-language features we are now able to express composite transformations, respectively state compound processes, on objects, respectively state variables. The examples of this part take on a flavor distinct from that of the examples of part II. There the examples were explicitly tied to the individual subjects being covered. Explicit examples using the constructs formally introduced in part III are given already in part II. Instead the examples of part III tend to be rather more comprehensive, illustrating several aspects of abstract modeling simultaneously.

In part IV we wrap up the story on function definitions and abstract models.

This part is not comprehensive. It relies on the subject being already, albeit partially covered in parts II III. Part $V$ ties up loose ends concerning elementary data types.

In addition to the above contents survey we advise the reader to carefully study the contents listing in order to ascertain the logical structure of this primer. 
PART II DOMAINS, OBJECTS \& OPERATIONS

Function definitions describe transformations on data objects. As such the defined functions apply to a domain of objects and yield objects of the range. The specific transformation is expressed, in terms of forms (constructors) denoting objects, operators and structured combinators denoting operations on objects.

The above paragraph serves the purpose of delineating the three cornerstones of this part: the story on meta-language means of defining domains of objects, representing constructed objects and operations on objects. Most sections will be structured accordingly. These will have basically three subsections. One will outline and exemplify how domains are defined, i.e. represented by means of so-called logical type- (or, as we shall prefer to call them, domain) expressions. Another will outline and exemplify how instances of objects of the domain are constructed. Finally a third will outline and exemplify expressions formed around operators denoting operations on objects. This last is split into two subsections: one on primitive operations, the other on combinators.

Each section dealing with a composite abstract data type will be introduced by examples whose purpose is to capture the essence of the objects under discussion, thus motivating you right into the rather formally structured parts. And each section will be concluded with larger examples.

In bringing examples we intend to illustrate basic principles of abstract modeling using the meta-language. The examples take care to use the meta-language in "good style".

[In this way you will also be introduced to representational- \& operational abstraction; to applicative- \& imperative programming; and, in a few places, to configurational- \& hierarchical abstraction.] 


\section{$\underline{2} \underline{S E T S}$}

\section{Example 1:}

The situation which we wish to abstractly define is perhaps rather 'childish'. We are given a set of classes, each class consisting of a set of students. Let students be abstracted by their school registration code. Let the domain of all such codes be student. Then:

$$
\text { Class }=\text { Student-set }
$$

is an abstract syntax rule whose left-hand side is an identifier, and whose right-hand side is a domain expression. It describes a class as being a set of (distinct) students. The equation gives the name class to a domain, and this domain is defined, by student-set, to be the domain of finite subsets of student.

Let suitably decorated $s$ 's and $c^{\prime}$ s denote respectively students and classes, i.e.:

$$
\begin{aligned}
& \text { is-student(s) } \\
& i s-C \text { Lass (e) }
\end{aligned}
$$

Now:

$$
\left\{s_{1}, s_{2}, \ldots, s_{n}\right\}
$$

is a set constructor expression. It denotes a class.

To test whether a given student, $s$, attends a given class, $c$, we write:

$$
s \in c
$$

-- which we read: $s$ is a member of $c$. If $s$ is not a member of $c$, then the expression is false, otherwise true. If we call the function that tests class recordings for is-in-class then:

$$
i s-i n-c \operatorname{Lss}(s, c)=s \in c
$$

is a function definition. To compute the students attending two classes, $c_{1}$ and $c_{2}$, we write: 
$c 1 \cap c 2$

-- which we read: the intersection of $c_{1}$ and $c_{2}$, i.e.: the set of students common to both $c_{1}$ and $c_{2}$. If no students attend both $c_{1}$ and $c_{2}$, then:

$$
c_{1} \cap c_{2}=\{\}
$$

their intersection is empty. The students following either $c_{1}$ or $c_{2}$ or both is denoted by:

$$
c_{1} \cup c_{2}
$$

-- which we read: the union of $c_{1}$ and $c_{2}$. To record that a class, $c$, is diminished by the departure of a student, $s$, we write:

$$
c \backslash\{s\} \quad \text { or } \quad c-\{s\}
$$

-- which we read as: the complement of $s$ with respect to $\{s\}$, or -which might be more to your liking -- the set c subtracted $\{s\}$. We use the notation - and - interchangeably, although not in this primer, where - is preferred. To record that a class, $c$, is augmented by the entry of a student, $s$, we write:

$$
c \cup\{s\}
$$

The number of students in a class is:

carde

-- for cardinality. 


\subsection{Defining Domains of SET objects}

Let $A$ be the name of a class of objects i.e. a domain. To define the class of objects which are finite sets of $A$ objects (i.e. finite subsets of $A$ ), we use the domain expression operator -set: $A$-set.

\section{Example 2 :}

The domain whose objects are finite sets of integers, i.e. finite subsets of INTG, is definable as:

$$
\text { INTG-set }
$$

Call this domain IS. Then:

$$
I S=I N T G-s e t
$$

is an abstract syntax rule formally defining this name. Then the domain whose objects are finite sets of (potentially overlapping) finite sets of integers is definable as:

$$
\text { IS-set, or: (INTG-set)-set }
$$

\section{Example 3 :}

Let $R$ represent the domain whose objects are abstractions of what, in a file system, is otherwise thought of as records. If files of such a system are, or can be, unordered, finite collections of distinct records, then: 
is an abstraction of the class of files. The domain $R-s e t$, if $R$ is an infinite class, is also infinite -- its objects are, however, all finite sets. The form $R-s e t$ is a domain expression.

The file system thus modeled may seem a bit contrived. That is: you may never wish to design such a system. Note that at this stage the system has no notion of sequential files or keyed records. The examples, when expanded in subsequent sections will, however, appear more 'realistic'.

\subsection{Representing Instances of $S E T$ Objects}

\subsubsection{Explicit Enumeration}

Given distinct objects $a_{1}, a_{2}, \ldots, a_{n}$ which are all $A$ objects, the expression:

$$
\left\{a_{1}, a_{2}, \ldots, a_{n}\right\}
$$

is said to be an explicit enumeration of a set, which denotes an object of $A-s e t$.

\section{Empty Set}

-- is denoted by:

\section{$\underline{2.2 .2}$ Implicit Enumeration}

Given a function $F: D \rightarrow A$, where $D$ denotes some logical type (i.e. an arbitrary domain); and a predicate $P: D \rightarrow B O O L$, the expression:

$$
\{F(d) \mid P(d)\}
$$


is said to be an implicit set enumeration, and then denotes an object of $A-s e t$. Since $A$ could be any logical type expression the above describes how arbitrary sets may be represented. The implicit set constructor expression can be read as: The set of objects $F(d)$ such that the predicate $P(d)$ holds. Thus we read I as 'such that!

\section{Examples $\underline{4}-\underline{5}-\underline{6}$ :}

The constructor expression:

$$
\{\{1,3,5,7,9\},\{2,5,11,13,15\}, \ldots,\{\}, \ldots\{2,1,8\}\}
$$

denotes an object in (INTG-set)-set whose element sets, in this example, are not disjoint.

Let $r_{1}, r_{2}, \ldots, r_{n}$ denote distinct objects of $R, i . e$. be abstractions of records, then:

$$
\left\{r_{1}, r_{2}, \ldots, r_{n}\right\}
$$

is (an abstraction of) a file, i.e. an object in R-set.

Let $F_{r}$ be a total function from records into records, i.e.: type: $F_{r}: R \rightarrow R$, and let $f$ denote a file, e.g. the above, then:

$$
\left\{F_{n}(r) \mid r \in f\right\}
$$

denotes a file derived from $f$ having each of the records of $f$ processed by $F_{r}$.

\subsection{SET Operations:}

The following special SET operations are defined: 


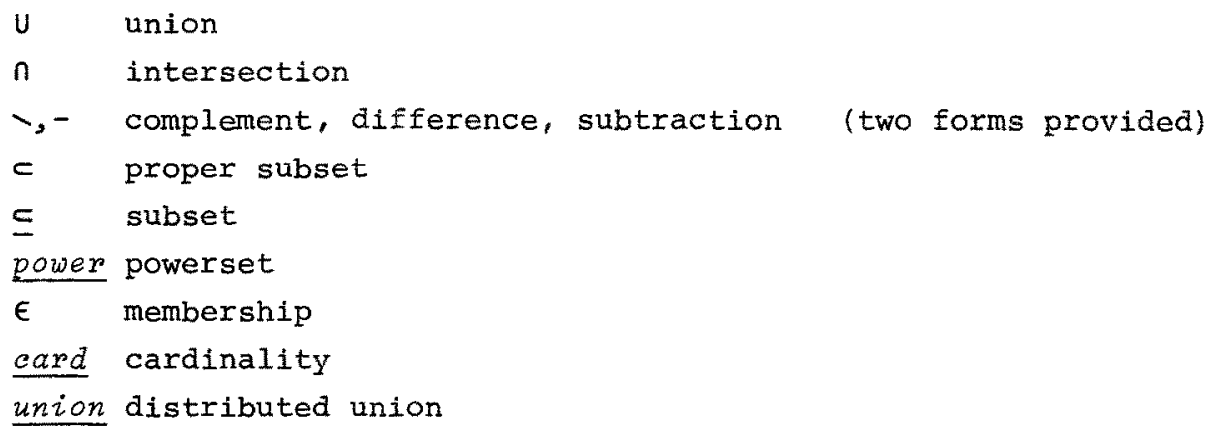

Each of these will now be individually and quite informally explained.

It is assumed below that set, set1, set2 denote sets and setsets a set of sets.

setiUset2 denotes the set of those objects which are either in set1, or in set2, or in both.

setinset 2

set $1>\operatorname{set} 2$

set.lcset 2

set 1 cset 2

power set

$o b j \in$ set

card set denotes the set of objects which are both in set 1 and sets.

denotes the set of objects which are in setl but not in set2.

denotes (the BOOLean truth value) true if all members of set1 are in set2 and there is at least one member of set2 not in set1, otherwise false.

denotes (the BOOL ean truth value) true if all members of set1 are in set2, otherwise false.

denotes the set of all finite subsets of set.

denotes (the BOOL ean truth value) true if the object denoted by obj is a member of set, otherwise false.

denotes the (Natural) number of members of set. Read as cardinality.

union setsets denotes the set consisting of all the objects of all the sets being elements of setsets. 
The set operations applied to anything other that sets are undefined.

\section{Examples $7:$}

Let $f$ denote a file, i.e. be in $R$-set; let $r$ and $r_{i}$ denote records (i.e. both be in $R$ ). The operator-operand expressions and constructs:

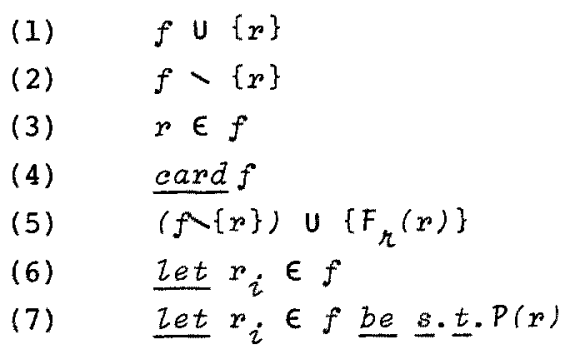

express typical set manipulations. These pure expressions could be used to provide a model of the following typical operations on files: (1) writing a (most likely new) record to a file; (2) deleting a record -- most likely, but necessarily, contained in a file -- from that file; (3) asking whether a given record is in a file; (4) asking for the number of records in a file; (5) updating a record in a file to a new record, i.e. replacing it -- with the possibility that it might not already be in the file, in which case $(1 \sim 5) ;(6)$ reading an arbitrary record from a file assumed not to be empty; and (7) reading an almost arbitrary record, namely one further satisfying the property expressed by the predicate function $P$.

Let $f l$ and $f^{2}$ denote files, i.e. both be in $R-s e t$, then:

$\begin{array}{ll}\text { (8) } & f 1 \cap f 2 \\ (9) & f 1 \subseteq f 2 \\ (10) & f 1 \subset f^{2} \\ (11) & f 1=f^{2} \\ (12) & f 1 \neq f 2\end{array}$


might be reasonable abstractions of the following, slightly hypothetical, operations among files: (8) collecting, into a file, the records common to two files; (9) asking whether all records of one file are contained in another file; (10) -- and, in addition to (9) -asking whether some records of the latter are not in the former; (11) asking whether two files are identical; or (12) different:

The expression:

$$
f^{1} \cup f^{2}
$$

is a generalization of (1), in that $\{r\}$ there denotes a singleton file, i.e. a file of exactly one record. (13) can be understood as the merge of the records of two files. The type of the object denoted by (13) is a file.

\section{$\underline{2.4}$ SET-oriented Combinators}

The following combinators are applicable to SET objects:

$$
\begin{aligned}
& \text { Let objE Set } \\
& \text { let } o b j \in \text { Set be s.t.t. P }(o b j)
\end{aligned}
$$

which you have already seen applications of, and:

$$
\text { for alz obje set do } s(o b j)
$$

The let clauses occur in expression- or statement blocks:

$$
\frac{(\text { Let }}{c(o b j} \in \text { Set } \ldots \text { in }
$$

where $C$ represents either an expression or a statement. The for all clause is a statement, and so must $s$ be.

For the general meaning of let clauses we refer to section 10.1. The specific let clauses shown above bind the identifier obj to an arbitrary, etc., member of the set, or domain, set, anywhere in $c$ where obj occurs free. 
Note that Set in let clauses may either be an expression denoting a finite set; or a domain-expression, potentially denoting infinite sets.

In the for-alz statement set must however be restricted to denote a finite set, in particular: the expression: set must not be a domain expression.

The meaning of the for-all clause is given here, but more systematically repeated in section 9.2.5. Let set denote the finite set whose $n$ objects we may arbitrarily name: $i d_{1}, i d_{2}, \ldots, i d_{n}$, where no $i d_{j}$ is free in $S(i d)$; then:

$$
/ /\left\{s\left(i d_{2}\right), s\left(i d_{2}\right), \ldots, s\left(i d_{n}\right)\right\}
$$

is a sufficient transliteration of the for-alz statement into a quasiparallel 'compound' statement, each of whose statements, $S(i d)$, arises from $S(O b j)$ by replacing all free occurrences of obj in $S(o b j)$ by $i d$. For the meaning of compound statements rely on your intuition, or look it up in section 10.4. Since the set element naming was arbitrary one can permute the above statements arbitrarily.

\section{Example 8:}

The constructor expression:

$$
\left\{F_{r}(r) \mid r \in f\right\}
$$

is an applicative expression -- provided $F_{r}$ does not rely on any state component. An imperative analogue can be achieved by means of the for-all statement:

$$
\begin{aligned}
& \text { del \{ile }:=\{\} \text { type } R \text {-set } \\
& \text { (for alz } r \in f \text { do } \\
& \text { bile := ébile } \cup\left\{F_{r}(r)\right\} \text {; } \\
& \text { c file) }
\end{aligned}
$$


The above can be seen to be a process-oriented abstraction of parts of a file manipulation system architecture. The global variable bile is initialized to the empty file, \{\} , of no records; and fixed to contain only finite sets of distinct records (type $R-s e t)$. File now denotes a constant reference, to an $R$-set object, $i . e$, an object in ref $R$-set; with elile denoting the dynamic contents at that reference, i.e. value of the file variable.

\subsection{Further Examples}

To sharpen your understanding of set manipulations we now bring further examples.

\section{Example 9:}

The problem which we wish to abstractly define is that of recording equivalence classes. A set (e.g. sas) consisting of disjoint sets (e.g. as 1, as2,..., asn) of (e.g. A) objects is said to define (or, which is the same, to 'realize') an equivalence relation. In fact we call the member sets $(a s 1, \ldots)$ for equivalence classes. The set of equivalence classes thus is a partitioning of the union of all the (A) objects of the equivalence classes. Given one partitioning and an $(A)$ object, supposed to be a member of an equivalence class, we wish to inquire whether it is indeed in some equivalence class of that partitioning. (We call the predicate which tests for this isRecorded.) As another subproblem we wish, given a partitioning and two 'recorded' $(A-)$ objects, $(a 1, a 2)$ to generate a new partitioning as follows: If the two $A$ objects are recorded in the same equivalence class, then the result partitioning is the same as the argument partitioning. If the two $A$ objects are recorded in distinct equivalence classes, then the result partitioning is as the argument partitioning except for the collapse (union) into one memberset of the two sets of which al and $a 2$ are respective members. (We call the new equivalence class generator function for enter). 


$$
\begin{aligned}
& E Q=B-\operatorname{set} \\
& B=A-\operatorname{set} t
\end{aligned}
$$

i.e.:

$$
E Q=(A-\operatorname{set})-\operatorname{set}
$$

2 $i s-w f-E Q(s a s)=$

$$
(\forall a s 1, a s 2 \epsilon s a s)(a s 1 \neq a s 2>a s 1 n \alpha s 2=\{\})
$$

3

$$
\text { isRecorded }(a, s a s)=
$$

$$
\text { ( } a \in \text { union sas) }
$$

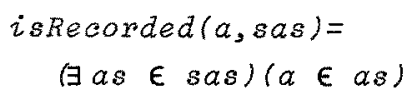

where we gave two versions of isRecorded.

4

.1

.2

5

.1

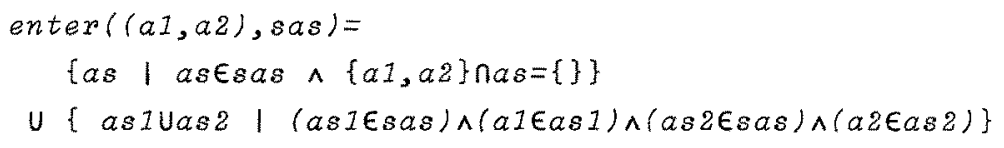

1. EQ names a domain of objects. These are finite sets of $B$ objects, with the latter being finite sets of $A$ objects -- hence an $E Q$ object is a set of sets of $A$ objects.

2. For such a set to be a partitioning, i.e. to be well-formed as per the intentions of defining the domain $E Q$, no two distinct members (of sas) may have any $A$ objects in common; or, which is the same, all membersets must be disjoint.

3. For an $A$ object, $a$, to be recorded, shall mean that the object $(a)$ is in some memberset, i.e. (3.3) that there is a memberset, as, of which it is an element. 
4. The two lines (4.1 \& 4.2) express what was stipulated above: the result partitioning is as the argument partitionings (4.1), except (4.2) -- etc.

The type clauses of the functions define the set of the input arguments, to the left of the arrows, and those of the results, to the right of the arrows. The arrows express that the defined functions are (indeed) (possibly partial $\stackrel{(\sim)}{\rightarrow})$ functions -- from the argument domains into the result domains.

\section{Example 10:}

As in the previous example, we deal with objects:

$$
S=(A-\operatorname{set})-\operatorname{set}
$$

-- but now not subject to any restrictions. To check whether an $S$ object, sas, is a partitioning we apply:

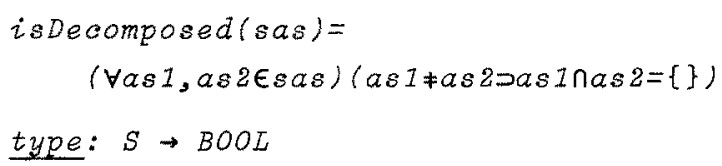

To decompose an $s$ object, sas, into the coarsest, i.e. smallest, having fewest elements, partioning of sas, we apply decompose(sas).

A possibly incomplete definition of a coarsest partitioning, given a set, sas, of potentially overlapping sets, goes as follows: Let $s a s^{\prime}$ be the result of decompose(sas). For all $a 1$ and $a 2$ that are members of member-sets of sas, $a 1$ and $a z$ are in the same member-set of sas' if-andonly-if (iff) for any subset, $\{a s 1, a s 2, \ldots, a s n\}$ of $s a s, a 1$ is in all asi, for $i \in\{1: n\}$, iff $a 2$ is in all $a s i$ (for $i \in\{1: n\}$ ); and only such $a$ 's are in member-sets of sas' which are similarly in sas, i.e.: union sas = unionsas'. 
Sometimes a picture is worth quite a few words:
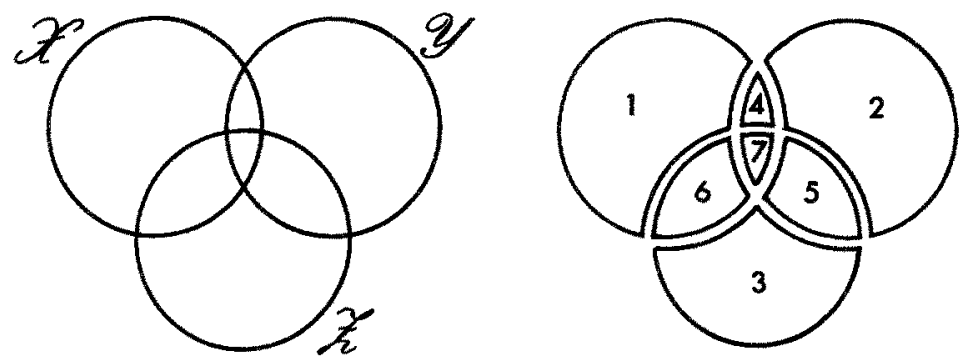

To the left is some sas $(\{x, y, z\})$, to the right is its corresponding sas': the digits 1-7 index the corresponding forms in the right-hand side expression below:

$$
\begin{aligned}
\text { decompose }(\{x, y, z\})= & \{x \backslash(y \cup z), y-(x \cup z), z \backslash(y \cup x), \\
& (x \cap y) \backslash z,(y \cap z) \backslash x,(x \cap z) \backslash y, \\
& x \cap y \cap z\}
\end{aligned}
$$

Here is a formal definition of the function:

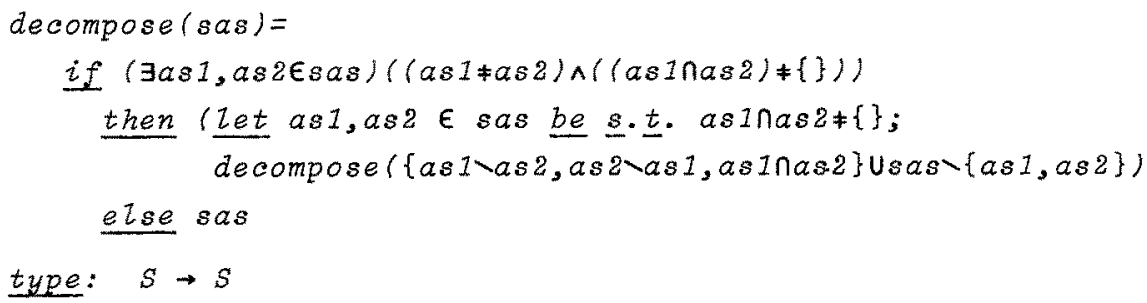




\section{TURLES}

\section{Example 11:}

The problem with which we wish to illustrate, before going into a more systematic coverage, the meta-language abstract data type of TUPLES, is perhaps not a very abstract one. We are to compute the first $k$ rows of the PASCAL triangle; informally:

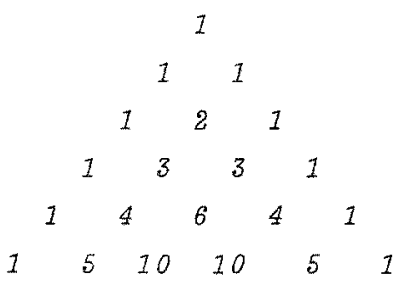

etc.. So we define two functions: one computing the $i$ 'th row; another putting the first $k$ rows together. The second function invokes the first.

$$
\begin{aligned}
& \text { type: row: } N_{1} \rightarrow N_{1}{ }^{+} \\
& \text {type: } \operatorname{tri}: N_{1} \rightarrow N_{1}^{++}
\end{aligned}
$$

row takes a positive, non-zero integer, i.e. a natural number larger then 1 , and produces a tuple of such numbers; $t x i$ takes a non-zero natural number and produces a tuple of as many tuples of natural numbers. $N_{1}^{+}$stands for the domain of tuples of natural numbers; $N_{1}{ }^{++}$ for tuples of tuples of these numbers. An implicit way of specifying row and tri would e.g. define their pre- and post-conditions, i.e. the conditions that input (alone) must satisfy, respectively the conditions that input and output (together) must satisfy:

type: pre-row: $N_{1} \rightarrow$ BOOL

type: post-row: $N_{1} \mathrm{~N}_{1}^{+} \rightarrow$ BOOL

type: pre-tri: $N_{1} \rightarrow$ BOOL

type: post-tri: $N_{1} N_{1}{ }^{++} \rightarrow B O O L$ 
In particular:

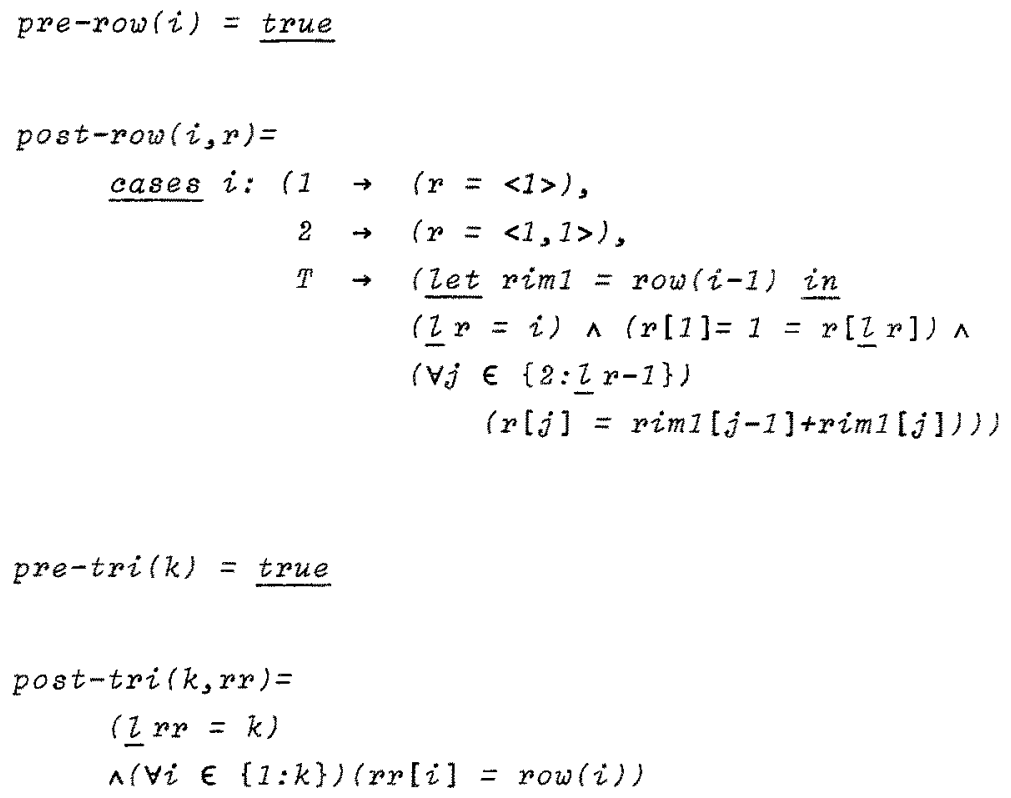

In the notation of the meta-language, tri(6) would be presented as:

$$
\langle\langle 1\rangle,\langle 1,1\rangle,\langle 1,2,1\rangle,\langle 1,3,3,1\rangle,\langle 1,4,6,4,1\rangle,\langle 1,5,10,10,5,1\rangle\rangle
$$

Giving the above implicit definitions of row and tri almost amounts to giving their explicit counterpart. Instead of doing this, let us "read" the pre- and post-'s:

The pre of row is always true (for $i>0$ ). The post of row says: if $i=1$ then the resulting row $x$ is just the tuple of length 1 whose only nember is a 1 . If $i=2$ then $r$ is a 2 -tuple both of whose elements are I's. In general, the length of $r$ is $i$, its first and last elements are both 1 's, and, for $i>2$, the elements of $x$, i.e. of row( $i)$, are related to elements of row $(i-1)$ as follows: let $j$ be any index into $r$ exclusive of the first and last, then the $j$ 'th element of $r$ is the sum of the $j-1$ 'st and $j$ th elements of row $(i-1)$.

The computation specified below computes $t r i(k)$ by imperative means: 


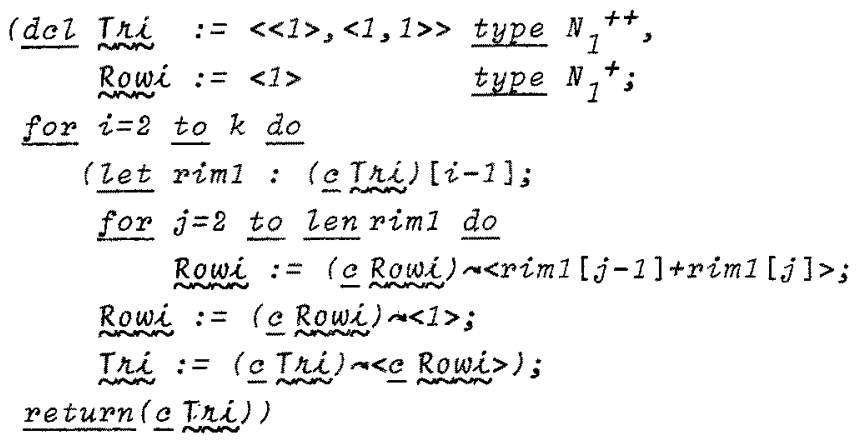

\subsection{Defining Domains of TUPLE Objects}

Let $A$ be the name of a class of objects. To define the class of objects all of whose members are finite length tuples whose elements are $A$ objects we use either of the domain expression operators: * or ${ }^{+}$.

\footnotetext{
$A^{*} \quad$ denotes the infinite domain of finite length tuples all of whose elements are in $A$. The 0 -length tuple, $<$, is in $A^{*}$ -- for any $A$ !
}

$A^{+}$denotes the infinite domain of finlte non-zero length rUPLEs, all of whose elements are in $A$. Thus $<$ is not in $A^{+}$.

\section{Example 12:}

Let $G$ denote a class of objects, i.e. a domain, abstracting the fields of a record, with records consisting now of a finite number of such ordered fields, then:

$$
G^{*}
$$

is a domain expression abstracting the class of records. Call this $R$, i.e.:

$$
R=G^{*}
$$


Now let files be ordered, finite length sequences of one or more records. Then:

$$
R^{+} \text {or }\left(G^{*}\right)^{+}
$$

are equivalent domain expressions abstracting the class of files. In the latter expression, the parentheses are solely used for grouping -you could, of course, omit parentheses here: $G^{*+}$. If you so wish you can likewise name the file domain:

$$
F t=R^{+}
$$

In this example we did not retain the view that files consisted of collections of distinct, unordered records. Had we done so:

$$
\begin{aligned}
& F s=R-\operatorname{set} \\
& R=G^{*}
\end{aligned}
$$

or:

$$
E s=\left(G^{*}\right)-\text { set, or: } F s=G^{*}-\text { set }
$$

would have been applicable abstractions.

\subsection{Representing Instances of TUPLE Objects}

\subsubsection{Explicit Enumeration}

Given not necessarily distinct objects $a_{1}, a_{2}, \ldots, a_{n}$ which are all in $A$, the expression:

$$
<a_{1}, a_{2}, \ldots, a_{n}>
$$

denotes an n-tuple, i.e. an object of, or in: $A^{*}, A^{+}$(for $n>0$ ). 
Empty Tupple

-- is denoted by:

$<$

3.2.2. Implicit Enumeration - Part 1

Given a function $F: I N T G \rightarrow D$ the expression:

$<F(i) \mid 1 \leq i \leq n>$

denotes an $n$ tuple -- as above! So does:
$<F(i) \mid i \in\{1: n\}>$,
and
< G(d) $\mid i \in\{m: m+n-1\}>$,
etcetera,

where $G: D \rightarrow A$. Thus we shall not be too particular about the form of the "type-building" predicate as long as it is clear, to the readers of your formulae, what the size, i.e. length, of your tuple will be. Since $G(d)$ is independent of $i$ the $n$ tuple consists of identical $G(d)$ elements.

\section{Examples 13 :}

Let $g_{1}, g_{2}, \ldots, g_{m}$, and $g_{i j}$ for varying $i, j$ denote fields of a record, i.e. all objects in $G$, then:

$$
\begin{aligned}
& <g_{1}, g_{2}, \ldots, g_{m}>, \\
& <<g_{11}, g_{12}, \ldots, g_{1 x}>,<g_{21}, g_{22}, \ldots, g_{2 y}>, \ldots,<g_{n 1}, g_{n 2}, \ldots, g_{n z}>>
\end{aligned}
$$

are constructor expressions denoting objects in $G^{*}$, respectively $R^{+}$ That is: abstractions of (instances of) records, respectively files! 
Let $F_{g}, F_{r}$ be total functions from fields to fields, respectively records to records, 1.e.: type: $F_{g}: G \rightarrow G$, type: $F_{r}: R \rightarrow R$; and let $f, r$ denote a file, respectively a record; then:

$$
\begin{aligned}
& <F_{g}(r[i]) \mid 1 \leq i \leq 2 r> \\
& <F_{r}(f[j]) \mid 1 \leq j \leq i f>
\end{aligned}
$$

denotes a recora, respectively a file, derived from $n$, respectively $f$, having all its fields, respectively records, processed -- in any order -- by $F_{g^{\prime}}$ respectively $F_{r^{*}}$ Although to be dealt with more systematically below $r[i]$ denotes the $i$ 'th field of record $r$, with $\underline{r}$ denoting the length, in terms of not necessarily distinct, fields, of record $r$.

\subsubsection{Element ordering}

Sets are unordered collections of objects. Tuple elements are ordered. In:

$$
<a_{1}, a_{2}, \ldots, a_{n}>
$$

$a_{1}$ is the 1 st element, $a_{2}$ the 2 nd,..., and $a_{n}$ the $n$th element. In:

$$
\langle F(i) \mid i \in\{m: m+n-1\}\rangle
$$

the 1 st element is $F(m)$, the 2nd element is $F(m+1), \ldots$, and $F(m+k)$ for $0 \leq k \leq n-1$ is the $k+1-$ st element. In:

$$
\langle G \text { (d) } \mid i \epsilon\{m: m+n-1\}\rangle
$$

all elements are alike, and are $G(d)$-- with $n$ of them!

By:

$$
\langle o b j| o b j \in \text { set }>
$$


we mean a card set tuple of all distinct members of the assumed finite set set -- occurring in arbitrary order.

In general the form of an implicit tuple building constructor expression is:

$$
\langle F(i) \mid O(i) \wedge P(i)\rangle
$$

where $O(i)$ is an ordering predicate indicating, mainly through the natural ordering of integers $i$, the order of those elements $F(i)$, for $i$, for which the more general predicate $P(i)$ holds:

\subsubsection{Implicit Enumeration - Part 2:}

Let $P: \quad I N T G \rightarrow B O O L$ be a (total function) predicate then the expression:

$$
<F(i) \mid \text { iEintgset } \wedge P(i)>
$$

denotes an $Z$ tuple :

$$
<F\left(i_{1}\right), F\left(i_{2}\right), \ldots, F\left(i_{2}\right)>
$$

where :

$$
\begin{array}{ll} 
& \left\{i_{1}, i_{2}, \ldots, i_{2}\right\} \subseteq \text { intgset } \\
\wedge & i_{1}<i_{2}<\ldots<i_{2} \\
\wedge & P\left(i_{1}\right) \wedge P\left(i_{2}\right) \wedge \ldots \wedge P\left(i_{2}\right) \\
\wedge & \left(\sim \exists i \in i_{\text {intgeet }}\left\{i_{1}, i_{2}, \ldots, i_{2}\right\}\right)(P(i))
\end{array}
$$

In words: The ordering of the resulting tuple elements follow the natural ordering of the INTeGers. The length of the tuple will be the cardinality of that subset of the finite integer set, intgset, for which $P$ is satisfied. 


\section{Example 14-ㅡㅁㅗ:}

Although not illustrative of abstraction techniques, as we intend them, the following examples might help drive home the idea of implicit tuple constructions:

(1) Let fib denote the so-called fibonacci function, type: fib: $N_{0} \rightarrow N_{1}$ where $N_{0}, N_{1}$ denote the sets of natural numbers larger than or equal to 0 , respectively 1 . In particular think of: $f i b(0)=1, f i b(1)=1$, with $f i b(i)$ for $i \geq 1$ being $f i b(i-2)+f i b(i-1)$. Then:

$$
<f i b(i) \mid(m \leq i \leq n) \wedge \underline{o d d} f i b(i)>
$$

denotes a tuple of those of the $m^{\prime} t h, m+1$ 'st,..., up to, and including the $n$ 'th fibonacci number provided these are not even numbers, and $m, n \geq 0$ ? For $m=3$ and $n=11$ you would get:

$$
\langle 3,5,13,21,55,89\rangle
$$

i.e. the tuple of the $3 \mathrm{rd}, 4 \mathrm{th}, 6 \mathrm{th}, 7 \mathrm{th}, 9 \mathrm{th}$ and $10^{\prime}$ th fibonacci number. The type of the above expressions is : $N_{1}^{*}$

(2) The following pure expression constructs the pascal triangle between rows 1 and $k$ :

$$
\begin{aligned}
& <\operatorname{row}(i) \quad i \leq i \leq k \\
& \wedge \text { cases } i \\
& (1 \rightarrow \operatorname{now}(i)=\langle 1\rangle \text {, } \\
& 2 \rightarrow \operatorname{row}(i)=\langle 1,1\rangle \text {, } \\
& T \rightarrow \operatorname{row}(i)=\langle 1\rangle \\
& -(\operatorname{row}(i-1))[j]+ \\
& (\operatorname{row}(i-1))[j+1] \\
& \mid 1 \leq j \leq \text { Ien } \operatorname{row}(i-1))> \\
& \pi<1>\text { ) > }
\end{aligned}
$$

You are invited to compare this formulation with the impure expressionblock given as the last item of example 11 above. 


\subsection{TUPLE Operations}

The following special TUPLE operations are provided:

[pairwise] concatenation,

$\begin{array}{ll}\begin{array}{l}h, \frac{h d}{t} \\ \frac{t z}{2}\end{array} & \begin{array}{l}\text { head, } \\ \text { tail, } \\ \text { length, }\end{array} \\ \underline{e l e m s} & \begin{array}{l}\text { elements, } \\ \text { indices, }\end{array} \\ \frac{i n d}{[i],(i)} & \begin{array}{l}\text { index, selection, (two forms provided) } \\ \text { conc }\end{array} \\ + & \text { distributive concatenation (of tuple of tuples), } \\ & \text { replace. }\end{array}$

Each of these operators will now be individually, and quite informally explained. It is assumed below that tuple, tuple1, tuplez denoted TUPLEs and tuplelist a TUPLE of TUPLEs.

tuplel atuplez denotes the TUPLE whose first $\underline{Z}$ tuplel elements are those of tuple 1 in the given order: whose last $\underline{l}$ tuple 2 elements are those of tuplez, and in that order; and whose length is exactly the sum of the lengths of tuplel and tuplez.

Intuple denotes the 1st element of tuple. Taking the head of an empty tuple is undefined.

tuple denotes the tuple of all but the first element of tuple and otherwise in the same order as the elements of tuple. Taking the tail of an empty tuple is undefined.

Ituple denotes the length of the tuple, i.e. the Number of not necessarily distinct elements of tuple.

elemstuple denotes the SET of elements contained in the tuple. 


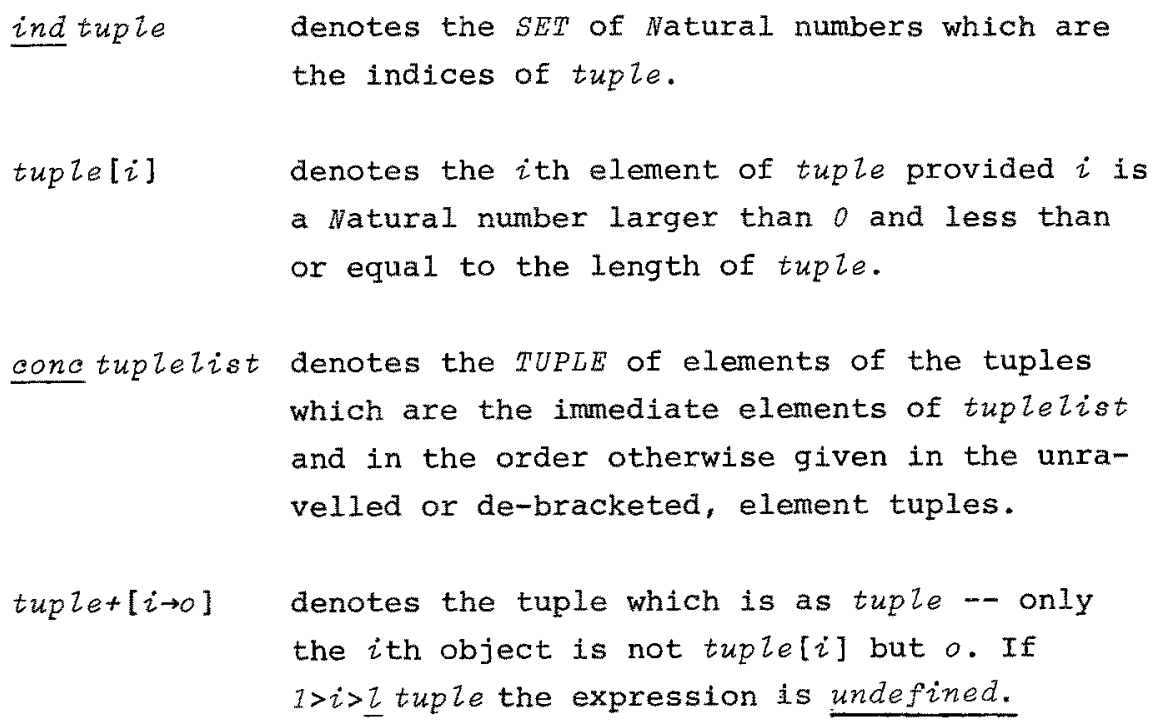

This last operation is (presently) only defined for a right operand singleton map (in $\left.\hat{N}_{1} \underset{\dot{m}}{O} O B J\right)$.

The tuple operations applied to objects other than tuples are undefined.

\section{Examples 16-17:}

Let VAL denote the class of values obtained during, i.e. as a result of partial, evaluations of expressions of some language, and let $S T A C K$ be an abstraction of stacks of these values.

$$
\text { STACK }=\text { VAL* }
$$

can then be considered a not too abstract, yet sufficiently implementation-independent, model of the domain of stacks. Let stkESTACK, $v \in V A L$ then:

<v>mst

hist, $\underline{h d} s t$

tst, $\underline{t 2} s t$ 
are correspondingly reasonable abstractions of the following typical operations on or facts about stacks: (1) pushing a new object "on top" of, or into the stack; (2) reading the top of the stack; (3) the resulting stack after a pop operation -- with (4) not abstracting any operation but only brought here to exemplify the empty, unused stack.

Reverting now to our examples around file systems, let possibly suitably decorated $f, r$ and $g$ denote files, records and fields respectively, i.e. objects in $F, R$ and $G$, then the expressions:

\begin{tabular}{|c|c|}
\hline$f \sim<r>$ & $p m<g>$ \\
\hline$\frac{h f, \quad h d f}{f[i]}$ & $\frac{h r,}{r[j]} \quad \underline{h d}$ \\
\hline$f^{1} \sim f^{2}$ & $r 1 m p 2$ \\
\hline$f+[i \rightarrow r]$ & $p+[j \rightarrow g]$ \\
\hline conef & \\
\hline If $f$ Zen $f$ & Zen $r$ \\
\hline
\end{tabular}

are reasonable abstractions of the following typical operations on files (left column) [and records (right column)]: (5) writing a record [field] to a sequential, even serial file [record]; (6) \& (7) reading the first, respectively the $i^{\prime}$ th record [ $j^{\prime}$ th field] of a file [record]; (8) chaining two files [records] together to form a new file [record]; (9) updating the $i$ 'th record [ $j$ 'th field] of a file [record]; (10) chaining all the records of a file together to form a record (:); and (11) inquiring about the current size of a file [record] in terms of its number of records [fields].

\subsection{TUPLE-oriented Combinators}

The following combinator:

$$
\text { for } i=m \text { to } n \text { do } s(i)
$$

is provided for the imperative handing of primarily lists of objects. In particular, let $t$ denote a tuple, then: 


$$
\text { for } i=1 \text { to lent do } s(t[i])
$$

is a typical statement. The integer valued bounds $m$ and $n$ must be statically determined. $S$ is any statement. See the doubly nested example of a use of the above iterative statement given as the last item of example 11 .

The meaning is given by the transcription:

$$
S(m) ; S(m+1) ; \ldots ; S(n)
$$

where $S(j)$ axises from $S(i)$ by substituting all free occurrences of $i$ in $S(i)$ by $j$. Since $j$ is a constant no collision with other free variables in $S(i)$ will occur.

\section{Example 1 18}

The constructor expression:

$$
<F_{r}(f[i]) \mid 1 \leq i \leq \underline{\operatorname{len} f}>
$$

is an applicative expression - provided $F_{r}$ does not rely on any state component. An imperative analog can be achieved by means of the for-to-do linearly iterative loop statement:

$$
\begin{aligned}
& \text { del kile: }=<\text { type } R^{*} \\
& \text { for } i=1 \text { to lenf do } \\
& \text { Liee }:=\text { (c kile) }<F_{n}(f[i])>\text {; } \\
& \text { c kile }
\end{aligned}
$$

The above can be seen to be a process-oriented abstraction of parts of a file handling system specification. The global variable file is initialized to the null tuple, <>, of no records; and fixed to contain only finite length sequences of not necessarily distinct records (type $R^{*}$ ). (icle now denotes a constant reference to an $R^{*}$ object, i.e. an object of ref $R^{*}$; with $\underline{e}$ dile denoting the dynamic contents at that reference. 


\section{$\underline{\text { MAPS }}$}

Example 19:

As a prelude to a systematic treatment of the subject of maps we are in this introductory example going to illustrate the abstraction of what could be an operating system directory in terms of recursively nested maps. In this, perhaps hypothetical operating system users are partially ordered, i.e. hierarchically structured -- and this is reflected in the design of the directory. With each user is associated a qualified name consisting of an ordered sequence of zero one or more resource identifiers. The overall system directory is a directory and a directory consists of a finite non-empty set of uniqueIy identified resources. A resource is e.g. either a line printer, a card reader, a display terminal, a file identifier or it is a directory.

Abstractly modeling we write:

$1 \quad D I R=R i d \vec{m} R E S$

$2 \quad R E S=L P|C R| D T|F i d| D I R \mid \ldots$

The resource- and file-identifiers are considered to be further unanalyzed, e.g. elementary, objects:

$$
\text { Rid }=\ldots, \quad \text { Fid }=\ldots
$$

We also do not have here bother to specify what line printers, card readers and display terminals are:

$$
L P=\ldots, \quad C R=\ldots, \quad D^{T}=\ldots
$$

The above two equations $(1,2)$ are examples of abstract syntax rules. The first defines the domain of finite domain maps from resource identifiers to resources, and names it $D I R$; the latter defines the domain of resources to be the union (1) domain of the domains listed to the right, i.e. in the definiens. Thus a particular resource, i.e. an object of the domain RES, is either an object in $L P$ (i.e. a line printer), or -- etc. 
If by suitably decorated lower case letter sequences analogous to the above domain names we mean to identify objects of respective classes, then the following is an expression of the meta-language denoting an object in DIR, i.e. an abstract "snapshot" of a directory:

$$
\begin{aligned}
& {\left[x i d_{1} \rightarrow z p_{1},\right.} \\
& \operatorname{rid}_{2} \rightarrow f i d_{2} \text {, } \\
& \mathrm{rid}_{3} \rightarrow \mathrm{Irid}_{4} \rightarrow \mathrm{Cr}_{4} \text {, } \\
& \operatorname{rid}_{5} \rightarrow d t_{5} \text {, } \\
& r i d_{6} \rightarrow\left[r i d_{7} \rightarrow{z p_{7}}\right. \text {, } \\
& \mathrm{rid}_{8} \rightarrow\left[\mathrm{rid}_{g} \rightarrow \mathrm{or} g\right] \text {, } \\
& \operatorname{rid}_{10} \rightarrow \text { fid }_{10} 11 \text {, } \\
& \left.x i d_{12} \rightarrow\left[r i d_{13} \rightarrow d t_{13}\right]\right]
\end{aligned}
$$

which we could informally picture:

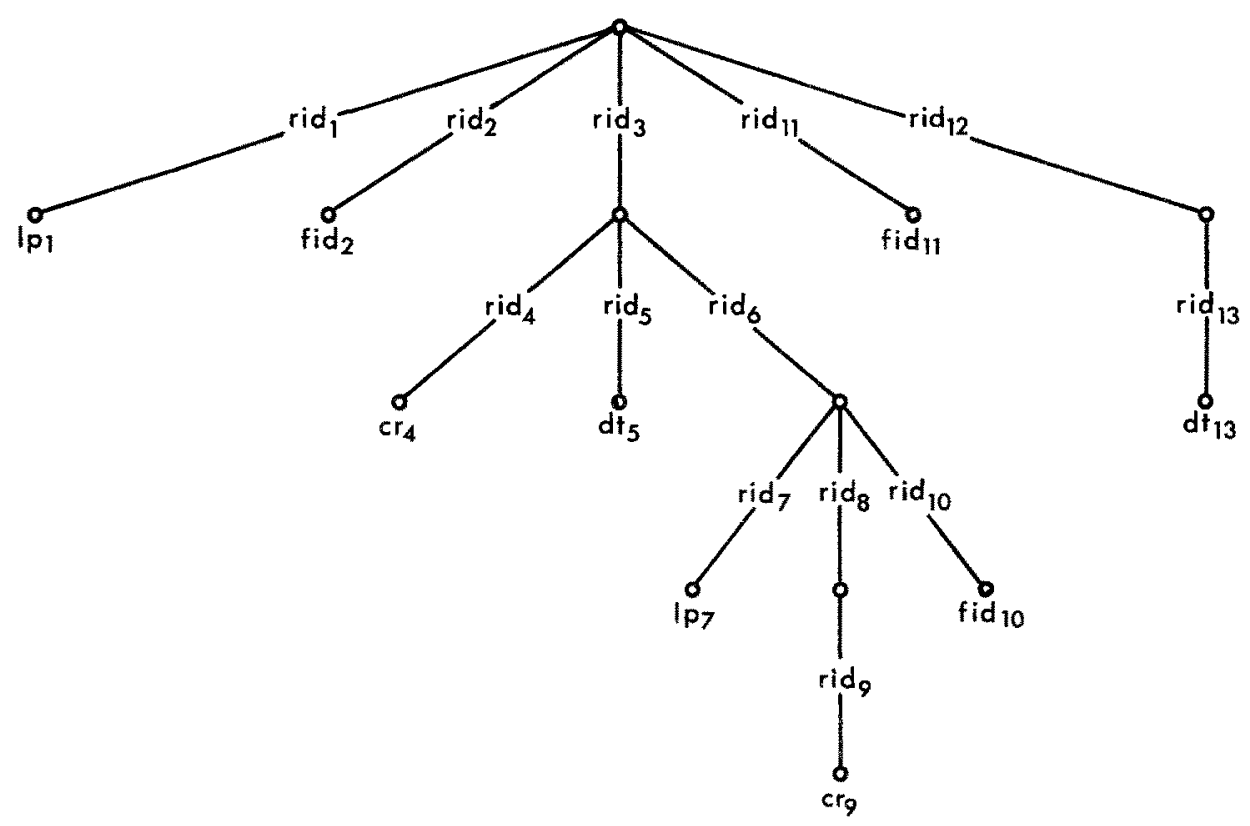


In the above hierarchical structure the labeling of edges (by rids) is usually understood to void any 'meaning' you may think that the left-to-right ordering of edges have.

Observe how, from the system-directory the qualified name $\left\langle r i d_{3}, r i d_{6}, r i d_{7}\right\rangle$ designates $l_{p}$. Qualified names designating directories are: \langle\rangle$,\left\langle r i d_{3}\right\rangle,\left\langle r i d_{12}\right\rangle,\left\langle r i d_{3}, r i d_{6}\right\rangle,\left\langle r i d_{3}, r i d_{6}, r i d_{8}\right\rangle$, and these are the names associated with users.

Resources associated with existing users may be allocated or freed: Let sys-dip stand for the system directory, qn for a name known to designate a direcotry in sys-dir, and let rid and res denote respectively the name by which the resource res is to be known in the directory designated by $q n$. The following function, when invoked by: catalog(sys-dir, qn, rid, res) accomplishes this:

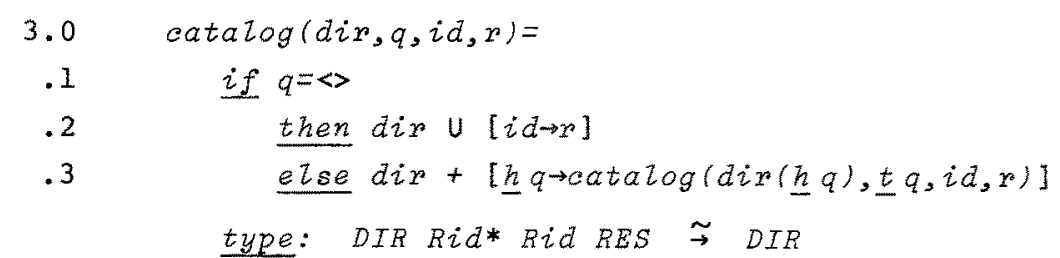

The reader is encouraged to apply this function to the above example.

The line: "dir $\cup[i d \rightarrow r] "$ reads: "join, or merge, as a new entry, the association of $i d$ to $r$, to the map $d i r "$. The last line reads: " $h q$, which is an Rid, designates a directory, $\operatorname{dir}(\underline{h} q)$, embedded in $d i r$. Replace this directory, $\operatorname{dir}\left(h_{q} q\right)$, with the one -catalog $(\operatorname{dir}(\underline{h} q), \underline{t} q, i d, r)$-- obtained by cataloging the resource $r$ named $i d$ in the subdirectory of $d i r(\underline{h} q)$ designated by $\underline{t} q:$ ".

Now it may be that the original qn did not designate a directory in sys-dir. Then the above function would go awry. So here is an improved version: 


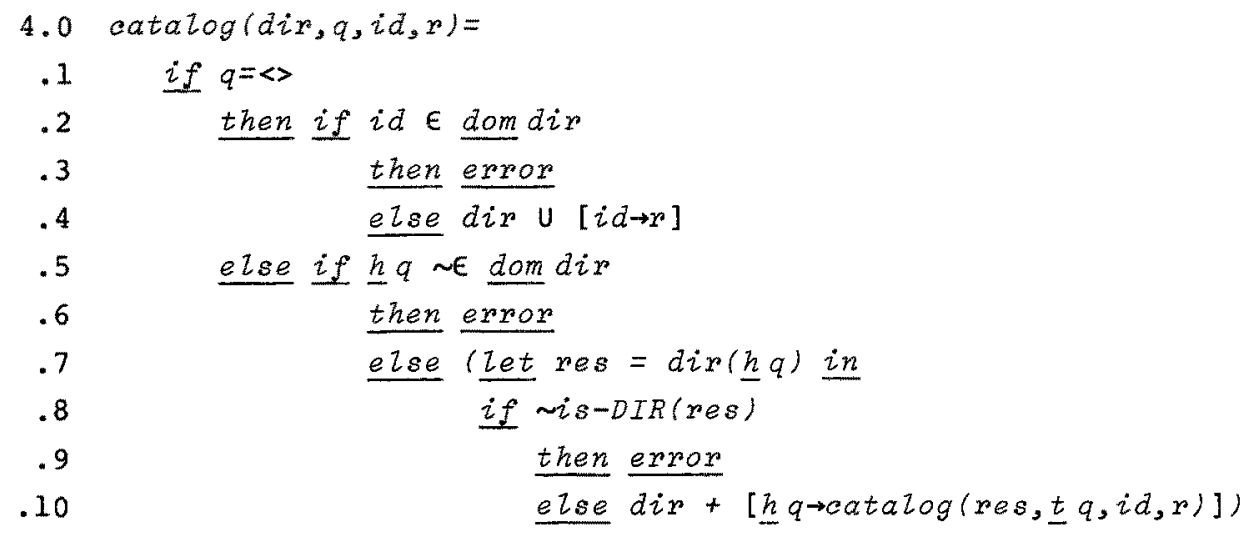

This function also makes sure that is is not already used in the directory designated by $q$. Thus dom dir denotes the domain, i.e. the set of resource identifiers used in dir. Observe finally that since a qualified name might designate either nonsense or a resource, res, other than a directory, i.e. a $D I R$, we test, is-DIR, that res is indeed a directory.

Further experiments with the above abstraction will be exhibited in section 4.5 . 


\subsection{Defining Domains of MAP objects}

Let $A, B, A_{i}$ and $B_{j}$ (for $i=1,2, \ldots, n$ and $j=1,2, \ldots, i$ ) be class names. To define the class of objects which are finite domain maps from (subsets of) $A$, respectively $A_{i}$, into $B$, respectively $B_{j}$, we use the domain expression operator $\vec{m}^{\text {: }}$

$$
A_{\vec{m}}^{B}, \quad A_{i} \vec{m}^{B} j
$$

\section{Examples $\underline{2} \underline{0}:$}

Let our file system now come of age! Let the file system consist of a set of uniquely named files; and let each file consist of a set of uniquely keyed records, i.e. the file may have its records randomly accessed by key. Let file names, keys and records presently be further unspecified. Then:

$$
\text { Key } \vec{m} R E C
$$

is a domain expression suitably abstracting our notion of files, provided key \& $R E C$ denote the domains of keys and records. If you wish to name this file domain, by e.g. FILE, then:

$$
F I L E=\operatorname{Key} \underset{m}{R} R E C
$$

is an example of an abstract syntax rule which lets $F I L E$ denote what Key $\vec{m}$ REC denotes! Now:

$$
\text { Fid } \vec{m} \text { FILE }
$$

or equally well:

$$
\text { Fid } \vec{m}(\text { Key } \vec{m} R E C)
$$

are domain expressions both suitable abstracting our ideas about the file system and files, again provided Fid denotes the domain of file names. 


\subsubsection{Defining MAP Domains -- Continued}

Then the logical type expression:

$$
\left(A_{1}\left|A_{2}\right| \ldots \mid A_{n}\right) \quad \vec{m}\left(B_{1}\left|B_{2}\right| \ldots \mid B_{2}\right) \quad l \geq_{n}
$$

denotes the class of objects whose members are finite domain maps from (subset of) the union class of $A_{1}, A_{2}, \ldots$ and $A_{n}$ objects into the union class of $B_{1}, B_{2}, \ldots$, and $B_{2}$ objects.

\section{Example 21:}

Now define keys and records to be either integers or characterstrings, respectively finite, non-zero length sequences of either integers or characterstrings -- presently with no constraints. Then, if INTG and QUOT ${ }^{+}$are suitable abstractions of integers and characterstrings respectively, we have that:

$$
\text { (INTG|QUOT } \left.{ }^{+}\right) \vec{m}\left(I N T G^{+} \mid Q_{U O T}^{++}\right)
$$

is a domain expression which defines any one file to contain both integers and characterstrings in its domain, i.e. as its keys; and sequences of both of these in its co-domain or range, i.e. as its records. Thus e.g. a given file may map 3 into $\langle\underline{A}, \underline{X}, \underline{H}\rangle,\langle\underline{P}\rangle$ into $\langle 2,4,9,11\rangle, 5$ into $\langle 3,5,7\rangle$, and $\langle\underline{Y}, \underline{Z}\rangle$ into $\langle\underline{I}, \underline{J}\rangle$.

\subsubsection{Defining MAP Domains - Continued}

The logical type expression:

$$
\left(A_{1} \vec{m} B_{1}\right) \underline{U}\left(A_{2} \vec{m} B_{2}\right) \underline{U} \ldots . \underline{U}\left(A_{k} \vec{m}^{B_{k}}\right)
$$

denotes the class of objects whose members are finite domain maps as above but with the restriction that $A_{1}$ objects, if at all mapped, i.e. In the domain, are mapped into $B_{1}$ objects, $A_{2}$ objects (...) into $B_{2}$ objects, $\ldots$, and $A_{k}$ objects into $B_{k}$ objects. We read the UMAP domain operator as a 'merge'-, rather than (as we did for 1) an 'either'-, union operation. 
Example 22:

If we wish to constrain integer keys to map into integer field records, and characterstring keys into records whose fields are characterstrings, then:

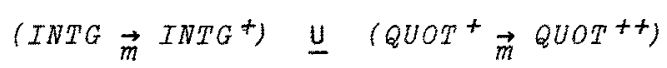

is a domain expression of the right kind for specifying the above:

\section{Defining MAP Domains -- Terminated}

The logical type expression:

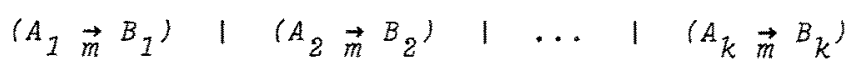

denotes a class of objects whose members are finite domain, partial maps from $A_{1}$ into $B_{1}$, or $A_{2}$ into $B_{2}, \ldots$ or $A_{k}$ into $B_{k}$. Thus a given member is either an $A_{1} \vec{m}^{B_{1}}$ object, or an $A_{2} \vec{m}_{2}^{B_{2}}$ object,..., or an $A_{k} \vec{m}^{B}{ }_{k}$ object.

\section{Example 23:}

If finally any one file should not mix integers and characterstrings in its key domain or record co-domain, then:

$$
\begin{aligned}
& \text { (INTG } \left.\vec{m} \text { INTG } G^{+}\right) \mid\left(\text {(INTG } \vec{m} \text { QUOT }{ }^{++}\right. \text {) | }
\end{aligned}
$$

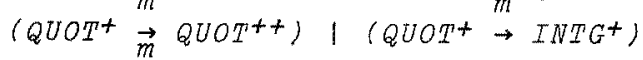

is a domain expression guaranteeing this: Note that the domain expression:

$$
\left(I N T G \mid Q U O T^{+}\right) \vec{m}\left(I N T G \mid Q U O T^{+}\right)^{+}
$$

permits any one record to have some fields being integers, others being characterstrings. If that is your ideas about records, this is a way in which you can so express it! 


\section{Notete :}

A map or a function from subsets of $A$ into $B$ is said to be a partial map, respectively partial function. It is quite common to deal with partial maps.

\section{Programming №t트:}

Defining a class of functions using the $\vec{m}$ operator shall express that such objects have their graph, i.e. argument-value associations, computed when defined. This is in contrast to using the $\rightarrow$ and $\underset{\sim}{\rightarrow}$ operators whose use in defining total and partial functions shall express that the objects are implicitly defined through some $\lambda$-expressions-like device, about which it can be said that the graph of the denoted function is not computed when defined -- in fact: is never computed -- and where, in addition, the function domain(s) may be infinite. For the story on such functions see section 5 .

\subsection{Representing Instances of MAP objects}

\subsubsection{Explicit Enumeration}

Let $a_{1}, a_{2}, \ldots, a_{d}$ be distinct $A$ objects, $a_{i 1}, a_{i 2}, \ldots, a_{i d}$ be distinct $A_{i}$ objects, $b_{1}, b_{2}, \ldots, b_{d}$ be not necessarily distinct $B$ objects, and $b_{j 1}, b_{j 2}, \ldots, b_{j d}$ be not necessarily distinct $B_{j}$ objects, then the constructor expressions:

$$
\begin{aligned}
& {\left[a_{1} \rightarrow b_{1}, a_{2} \rightarrow b_{2}, \ldots, a_{d} \rightarrow b_{d}\right]} \\
& {\left[a_{i 1} \rightarrow b_{j 1}, a_{i 2} \rightarrow b_{j 2}, \ldots, a_{i d} \rightarrow b_{j d}\right]}
\end{aligned} \quad \text { and }:
$$

denote maps from (subsets of) $A$ into $B$, respectively (subsets of) $A_{i}$ into $B_{j}$, i.e. in: $A \vec{m} B$, respectively $A_{i} \vec{m} B_{j}$. The constructor expression: 


$$
\begin{aligned}
& {\left[a_{11 \rightarrow b_{11}}, a_{12} \rightarrow b_{12}, \ldots, a_{1 m_{2}} \rightarrow b_{1 m_{1}},\right.} \\
& a_{21} \rightarrow b_{21}, a_{22} \rightarrow b_{22}, \ldots, a_{2 m_{2}} \rightarrow b_{2 m_{2}}, \\
& \ldots \\
& \left.a_{k 1} \rightarrow b_{k 1}, a_{k 2} \rightarrow b_{k 2}, \ldots, a_{k m_{k}} \rightarrow b_{k m_{k}}\right]
\end{aligned}
$$

denotes a map in:

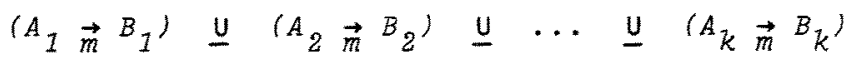

assuming of course that $a_{i j} \in A_{i}, b_{i j} \in B_{i}$ that is: that first subscripting index, $i$, binds to domain $A_{i}$ respectively $B_{i}$. And:

$$
\left[a_{x 1} \rightarrow b_{y 1}, a_{x 2} \rightarrow b_{y 2}, \ldots, a_{x m} \rightarrow b_{y m}\right]
$$

denotes a map in:

$$
\left(A_{1}\left|A_{2}\right| \ldots A_{n}\right) \quad \vec{m} \quad\left(B_{1}\left|B_{2}\right| \ldots \mid B_{2}\right)
$$

provided:

$$
a_{x i} E\left(A_{1}\left|A_{2}\right| \ldots \mid A_{n}\right) \text { and } b_{y j} \in\left(B_{1}\left|B_{2}\right| \ldots \mid B_{2}\right)
$$

\section{Empty Map:}

- is denoted by:

[]

\section{Example 2 2 :}

Let $f i d_{1}, f i d_{2}, \ldots, f i d_{l}$ denote, $i . e$. be abstractions of, distinct file names; let $k_{i j}$ etc. denote, $i . e$. be abstractions of keys distinct for fixed $i ' s ;$ and let $x_{i j}$ etc. denote, i.e. be abstractions of not necessarily distinct records. Then: 


$$
\begin{aligned}
& {\left[f i d_{1} \rightarrow\left[k_{11} \rightarrow x_{11}, k_{12} \rightarrow r_{12}, \ldots, k_{1 n_{1}} \rightarrow r_{1 n_{1}}\right],\right.} \\
& f i d_{2} \rightarrow\left[k_{21} \rightarrow r_{21}, k_{22} \rightarrow r_{22}, \ldots, k_{2 n_{2}} \rightarrow r_{2 n_{2}}\right], \\
& \ldots \\
& \left.f i d_{2} \rightarrow\left[k_{21} \rightarrow r_{21} k_{22} \rightarrow r_{22}, \ldots, k_{2 n_{m}}{ }^{\rightarrow r} I n_{l}\right]\right]
\end{aligned}
$$

is a schematized (..) snapshot of an object in Fid $\vec{m}$ (Key $\vec{m} R E C)$, provided $f_{i d_{i}} \in F i d, k_{i j} \in K e y$ and $r_{i j} \in R E C$.

If Key = INTG and REC = QUOT+t then:

$$
\begin{aligned}
{[3} & \rightarrow\langle\langle\underline{A}, \underline{Z}, \underline{B}\rangle,\langle\underline{Y}, \underline{C}\rangle,\langle\underline{X}, \underline{D}, \underline{W}, \underline{E}\rangle\rangle, \\
5 & \rightarrow\langle\langle\underline{V}\rangle\rangle, \\
7 & \rightarrow\langle\langle\underline{F}, \underline{U}, \underline{G}, \underline{\underline{T}}, \underline{H}\rangle,\langle\underline{S}\rangle\rangle]
\end{aligned}
$$

represents a file of three records, with keys 3,5 and 7 ; etc. Whereas keys of any one file need be distinct, records or any two keys of respectively distinctly names files need not be distinct:

$$
\begin{aligned}
{\left[f i d_{\alpha}\right.} & \rightarrow[2 \rightarrow<3,4,5>, 3 \rightarrow<3,4,5>], \\
f i d_{\beta} & \rightarrow[3 \rightarrow<3,4,5>, 2 \rightarrow<3,4,5>]] ;
\end{aligned}
$$

in fact, as the above example of a snapshot of a small file system shows, distinctly named files need themselves not be distinct.

\subsubsection{Implicit Enumeration}

Let $F: D_{d} \underset{\rightarrow}{\sim}$ and $G: D_{p} \underset{\rightarrow B}{ }$ denote partial functions, then:

$$
[F(d) \rightarrow G(x) \mid P(d, r)]
$$

is a map constructor expression denoting a map in $A \vec{m}^{B}$. P is assumed to be a predicate: $P: D_{d} D_{r} \rightarrow B O O L$. 
Let $F_{i} F_{1 n}, G_{j}, G_{l \ell}$ denote partial functions:

$$
\begin{array}{ll}
F_{i}: & D_{d} \stackrel{\sim}{\rightarrow} A_{i}, \\
F_{1 n}: & D_{d} \sim\left(A_{1}\left|A_{2}\right| \ldots \mid A_{n}\right), \\
G_{j}: & D_{x} \stackrel{\sim}{\rightarrow} B_{j} \\
G_{1 \ell}: & D_{r} \rightarrow\left(B_{1}\left|B_{2}\right| \ldots \mid B_{l}\right)
\end{array}
$$

and :

for $i=1,2, \ldots, n$ and $j=1,2, \ldots, z$. The expressions:

$$
\begin{array}{llll}
{\left[F_{i}(d)\right.} & \rightarrow G_{j}(r) & P(d, r)], & \text { and: } \\
{\left[F_{1 n}\right.} & \rightarrow G_{i \ell}(r) & P(d, r)]
\end{array}
$$

denote maps from (subsets of) respectively $A_{i}$ into $B_{j}$, and $\left(A_{1}\left|A_{2}\right| \ldots \mid A_{n}\right)$ into $\left(B_{1}\left|B_{2}\right| \ldots \mid B_{2}\right)$.

The expression:

$$
\begin{aligned}
& {\left[F_{1}(d) \rightarrow G_{1}(x) \mid P_{1}(d, x)\right]} \\
& U\left[F_{2}(d) \rightarrow G_{2}(x) \mid P_{2}(d, x)\right] \\
& \cdots \\
& \cdots \\
& U\left[F_{k}(d) \rightarrow G_{k}(x) \mid P_{k}(d, x)\right]
\end{aligned}
$$

where $P_{i}$ are predicates, as above, denotes a map in:

$$
\left(A_{1} \vec{m}^{B_{1}}\right) \quad \underline{\cup}\left(A_{2} \vec{m}^{B_{2}}\right) \quad \underline{U} \quad \cdots \quad \underline{U}\left(A_{k} \vec{m}_{k} B_{k}\right. \text {. }
$$

Example $\underline{2} \underline{5}$ :

Let $F_{r}, F_{6}$ denote total (for simplicity: pure, applicative) functions from records into records, respectively files into files. Let $s$ denote a file system, $f$ some file, $r$ some record; and, foregoing the next subsection (2.3.3) on operations, let dom $s$ and dom $f$ denote the set of filenames, respectively the set of keys, of the file systen -- respectively of a file. Then:

$$
\begin{array}{ll}
{\left[f i d \rightarrow F_{f}(s(f i d))\right.} & \mid f i d \in \underline{\text { dom } s]} \\
{\left[k \rightarrow F_{r}(f(k))\right.} & \mid k \in \underline{\text { dom } f}]
\end{array}
$$

and: 
denotes a file system, a file, and a file system. The former file system is derived from a given file system, $s$, by transforming each of its files; the latter by transforming each of its records individually. The file is derived from a given file, $f$, by transforming each of its records. Thus the above three implicit map constructor expressions may be reasonable abstractions of various file 'processing' programs. Observe how randomness of record and file 'positions' or 'accessing' is preserved by the unorderedness of 'fetching' files (fid) respectively records $(k, f(k), \ldots)$, for transformation.

\subsubsection{A Note on Scopes}

We have now seen three distinct meta-language constructs for implicit object constructions:

$$
\begin{aligned}
& \left\{F_{r}(r) \quad 1 \quad r \in f\right\} \\
& \text { < } F_{R}(f[i]) \mid \quad 1 \leq i \leq \underline{I}> \\
& {\left[k \rightarrow F_{h}(f(k)) \mid k \in \text { dom } f\right]}
\end{aligned}
$$

In all three, $F_{r}$ and $f$ were quantities defined outside these expressions; and $r, i$ and $k$ respectively, were bound variables, being defined and bound to the right of the builder combinator: 1 , and with a scope extending to the closest embracing pair of $\{\&\},<\&>$, respectively [ \& ]. The scope of these bound variables follow rules analog to those in ordinary programming languages: thus they extend to inner, i.e. nested expressions, including \{\}$,\langle>$ and [] based constructor expressions -- unless redefined in such inner forms, a practice which certainly can and should be avoided: The last file system building expression of the previous example section illustrates the doubly nested use of bound variables. So does:

\section{Example $2 \underline{6}$ :}

Let:

$$
\begin{aligned}
& A \vec{m}(B \vec{m} C) \\
& \text { (A B ) } \vec{m} C
\end{aligned}
$$


be domain expressions where we do not presently bother about $A, B$ and $C$. Then: for suitably decorated, and occasionally distinct, $a \in A, b \in B$ and $c \in C$ :

$$
\begin{aligned}
{[a 1} & \rightarrow[b 11 \rightarrow c 11, b 12 \rightarrow c 12] \\
a 2 & \rightarrow[b 21 \rightarrow c 21, b 22 \rightarrow c 22, b 23 \rightarrow c 23]]
\end{aligned}
$$

and :

$$
\begin{aligned}
{[(a 1, b 11)} & \rightarrow c 11,(a 1, b 12) \rightarrow c 12, \\
(a 2, b 21) & \rightarrow c 21,(a 2, b 22) \rightarrow c 22,(a 2, b 23) \rightarrow c 23]
\end{aligned}
$$

are examples of somehow 'equivalently' denoting expressions in that functions can be defined which converts between their objects.

Let, for ease of reference, the first domain be named $X$, the second Y:

$$
\begin{aligned}
& X=A \vec{m}\left(B \vec{m}^{C} C\right) \\
& Y=\left(\begin{array}{ll}
A B & \vec{m}^{C}
\end{array}\right.
\end{aligned}
$$

then:

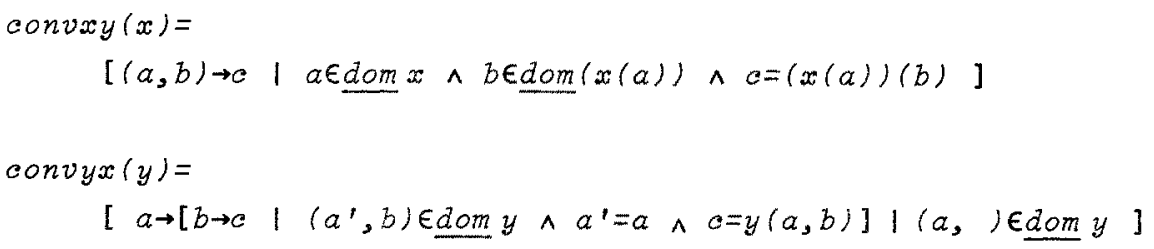

where:

type: convxy: $X \rightarrow Y$

type: convyx: $Y \rightarrow X$

In fact the two functions are each others' inverses. 


\subsection{MAP Operations}

The following special MAP operations are provided:

$\begin{array}{ll}U & \text { merge, } \\ + & \text { override, } \\ (\ldots, & \text { apply, } \\ & \text { restrict with, } \\ \text { I } & \text { restrict to, } \\ \underline{\text { dom }} & \text { domain, } \\ \underline{r n g} & \text { range, } \\ & \text { composition }\end{array}$

Each of these operators will now be individually, and quite informally explained:

map 1Umap2 denotes a MAP provided the domains of map1 and map 2 are disjoint, otherwise undefined. The denoted map maps domain elements of mapl into the same range elements as does map1, and domain elements of map 2 into the same range elements as does map 2 , and maps nothing else.

map1+map2 denotes the MAP which maps domain elements of map2 into the same range elements as does map2, and maps those domain elements of mapl which are not in the domain of map2 into the same range elements as does map 1, and maps nothing else.

map (a) denotes the range object into which map maps $a$. If $a$ is not in the domain of map the operation is undefined.

map-wset denotes the MAP which maps those domain elements of map which are not in the set wset into the same range elements as does map, and maps nothing else. 
mapltset denotes the MAP which map those domain elements of map which are also in tset into the same range elements as does map, and maps nothing else.

dom map denotes the set of objects which are domain elements of map.

rng map denotes the SET of OBJects which are the range elements of map.

map1"map2 denotes a MAP provided the range of map 2 is included, i.e. contained (ㄷ) in the domain of map1, otherwise the operation is undefined. The denoted map maps into those range elements of mapl which are mapped to by the domain elements of map1, mapped into, as range elements of map 2 from domain elements of map2. More formally, and in this case certainly more concisely:

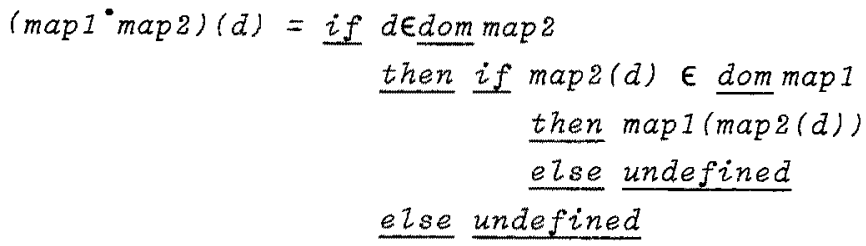

\section{Example 2 2 :}

Let $s, i d, f, r$ and $k$ stand for abstractions of file systems, file names, files, records and keys, then:

$\begin{array}{ll}s \cup[i d \rightarrow f] & f \cup[k \rightarrow r] \\ s+[i d \rightarrow f] & f+[k \rightarrow r] \\ s \backslash\{i d\} & f \backslash\{k\} \\ s(i d) & f(k) \\ i d \in \text { dom } s & k \in \text { domf } \\ f \in \text { rng }^{r n} & r \in \underline{r n g} f\end{array}$


may be reasonable abstractions of the following typical operations on file systems (left column) [and files (right column)]: (1) writing an entirely new file [record] into the file system [a file] -- in the sense of its name [key] not hitherto being one of a file [record] in the system [file]; (2) updating an entire file [a single record] of the system [a file]; (3) deleting an entire file [record] named $i d$ [keyed with $k$ ] from the system [a file]; (4) reading an entire file [a record]; (5) asking whether a file of a given name [a record with a given key] is in the system [a file]; and (6) inquiring whether a given file (i.e. its 'value', not name) [record ('value', not key)] is in the system [a file].

Let suitably decorated $i d^{\prime}$ s and $k^{\prime} s$ denote file names, respectively keys. Then:

$$
s\left|\left\{i d_{1}, i d_{2}, \ldots, i d_{n}\right\} \quad f\right|\left\{k_{1}, k_{2}, \ldots, k_{m}\right\}
$$

seems to be an acceptable abstraction for the operation of restricting the files of a system to those of the indicated names - i.e. deleting all other! [respectively deleting all those file records whose keys are different from $\left.k_{i}(2 \leq i \leq m)\right]$.

Combining the above operations:

$$
\begin{aligned}
& s+[i d \rightarrow s(i d) \cup[k \rightarrow r]] \\
& s+[i d \rightarrow s(i d)+[k \rightarrow r]] \\
& s+[i d \rightarrow(s(i d)-\{r\}] \\
& (s(i d))(k) \\
& k \in \underline{d o m}(s(i d)) \\
& r \in \underline{r n g}(s(i d)) \\
& s+\left[i d \rightarrow\left((s(i d)) \mid\left\{k_{1}, k_{2}, \ldots, k_{n}\right\}\right)\right]
\end{aligned}
$$

we get the right column equivalents, on a file system. 


\subsection{MAP-oriented Combinators}

The same combinator: the for-alz-do statement, as was defined for processing sets, is available for processes on maps, e.g.:

$$
\text { for all } d \in \text { dommap do } S(\operatorname{map}(d))
$$

\section{Exaranple 2 2}

We give the imperative variant of the last of the three implicit, applicative map constructions shown in the example 25 .

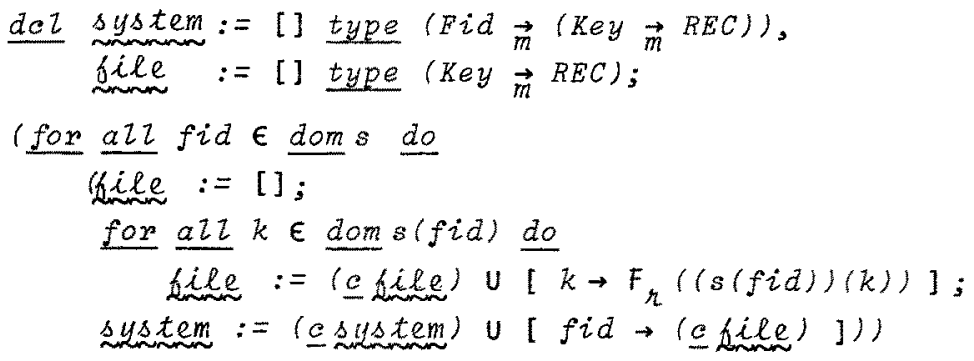

\subsection{Further Examples}

\section{Example 29:}

The introductory example of this section is now continued. We are now interested in defining a number of auxiliary functions applicable to the operating system directory. A function for catalogueing new entries, i.e. new resources, has already been shown. The dual function of uncatalogueing e.g. takes a directory and a non-null resource name and deletes the designated resource -- i.e. returns a directory in which the resource name is no longer a valid name: 
$1.0 \quad$ uncatatog $(2 i r, q)=$

.1

cases $q$ :

.2

$\langle i d\rangle \rightarrow \operatorname{dip}\{i d\}$

.3

$T \quad \rightarrow \operatorname{dir}+[\underline{h} q \rightarrow \operatorname{uncatalog}(\operatorname{dir}(\underline{h} q), \underline{t} q)])$

type: $D I R R i d^{+} \rightarrow$ DIR

Provided $q$ indeed does designate some resource in dir! when such is the case we can show that:

uncatalog $(e a t a \log (d i r, q, i d, r), q \pi<i d>)=d i r$

A function for testing whether a non-null resource name, $q$, does indeed designate something meaningfull in a directory, dir, is e.g. the following:

2

$$
\begin{gathered}
\text { isvalidrn }(\operatorname{dir}, q)=q \text { eresnms }(d i r) \\
\text { type: DIR Rid } \rightarrow \text { BOOL }
\end{gathered}
$$

where:

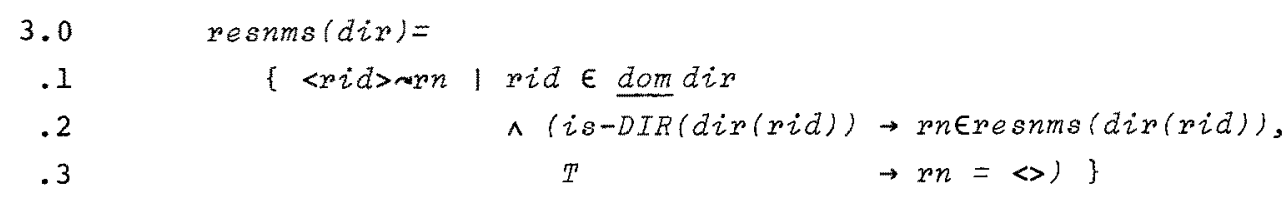

tupe: $\quad D I R \rightarrow R i d^{+}-$set

another function would be:

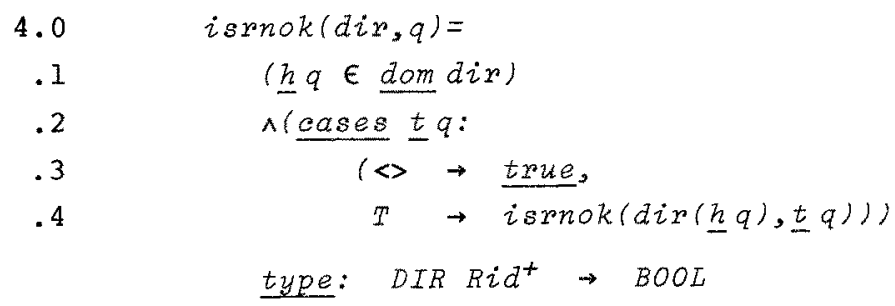

where we rely on the non-commutativeness, in the meta-language, of the boolean $A$ and $\checkmark$ operators. A function for retrieving a resource given its name could e.g. be expressed: 


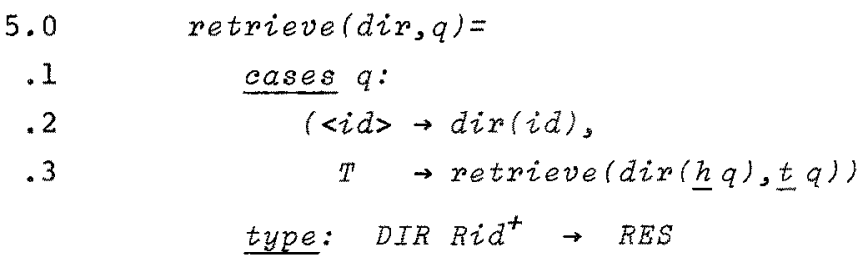

The domain expression $R i d \vec{m} R E S$ also defines the void map, [], from no resource identifiers to no resources, as part of its domain. In some of the above function definitions we have tacitly assumed that no (embedded) directory was empty. A predicate function for testing this well-formedness criteria not expressed by the domain expressions might e.g. look like:

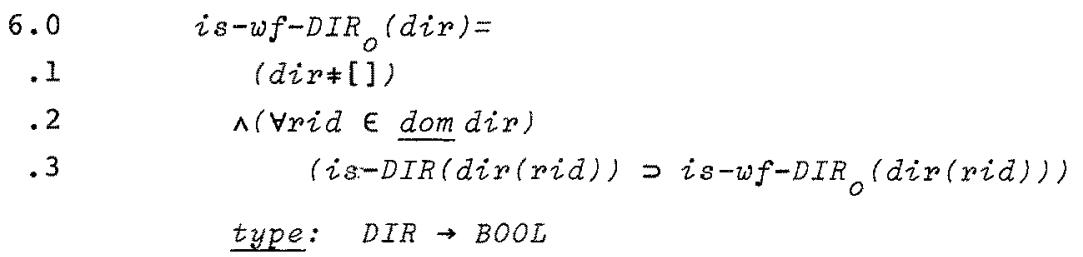

File identifiers are part of the directory. If we e.g. impose that no two distinct resource names, via designated file identifiers, "point" to identical files, then the above is-wf-DIR must be augmented:

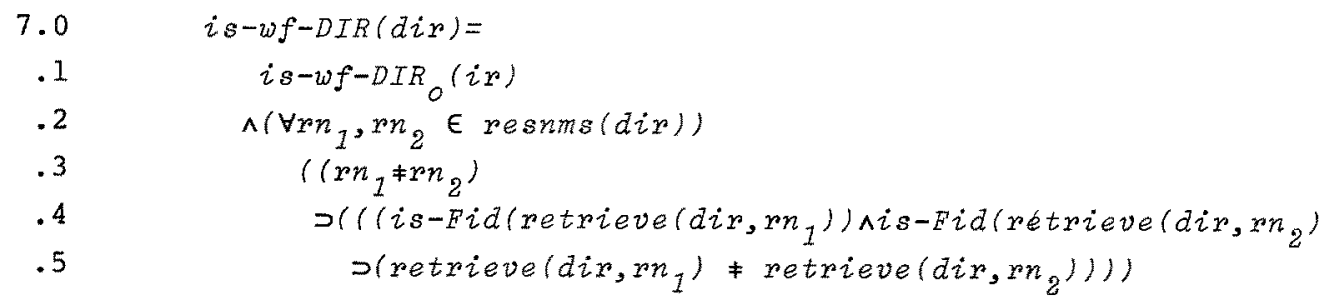




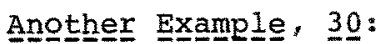

In this example we shall motivate the modeling of the scope-binding and variable concepts of block- \& procedure-oriented programing languages by means of so-called environments, respectively abstract stores. In subsequent examples, $(31,40,42,61)$, we shall then illustrate the semantics modeling of procedures and blocks of such languages. The introduction, into our realm of abstract modeling concepts, of environments and stores will now be argued in a few, short, illustrative, but not really complete steps. The notation used in the following for some arbitrary, realistic source languages notions of begin-end blocks and variable declarations and use has been deliberately chosen to coincide with the meta-languages notation for the same constructs. These will, however, not be formally introduced till section 10 .

The form:

$$
\left(\frac{d e z}{c(. .)} v:=\operatorname{expr} \text { type } D ;\right.
$$

is taken to represent some source languages block construct (with " (" equalling begin, and ")" the end). In the semantics of this source language $v$, which itself is an identifier, is to denote something constant. In fact: in this source-, as well as in our meta-, language, the denotation of identifiers is constant over their scope, i.e. does not change! The constant denotation of $v$ over the above block, i.e. its defining scope, is, rather concretely speaking, taken to be the location for: adaress) of the storage cell in which are to be held values, $c v$, of the variable $v$. Thus, if by $L O C$, we denote the abstract domain of storage locations, and by $O B J$, the values which can be held in those locations, then the domain of storages can be abstracted as:

$$
L O C \vec{m} \text { OBJ }
$$

We choose $\vec{m}$ since there will, at any point of execution of our source programs, only be a finite number of active variables, i.e. of allocated storage cells. Thus we abstract storages as unique associations, maps from locations to their values.

To keep track of the location that variable identifiers denote we introduce, speaking concrete again, a table, henceforth called an environment, in which is recorded the associations of variable names to their locations. 
Id $\vec{m}$ LOC

where e.g. $v \in I d$. Again the association is functional, and again the functional recordings are finite. Before argueing why we do not directly model storages as:

$$
\text { Id } \vec{m}^{\prime} O B J
$$

let us first see that we do indeed need the full use of the meta-language MAP concept, i.e. of the MAP operations. Simultaneously we exemplify 'snapshots' of enviromments, $\rho$, and stores, $\sigma$. The example is that of one simple block, containing the declaration of the variable named $i$, nested in an outer block, also containing the declaration of a identically named variable.

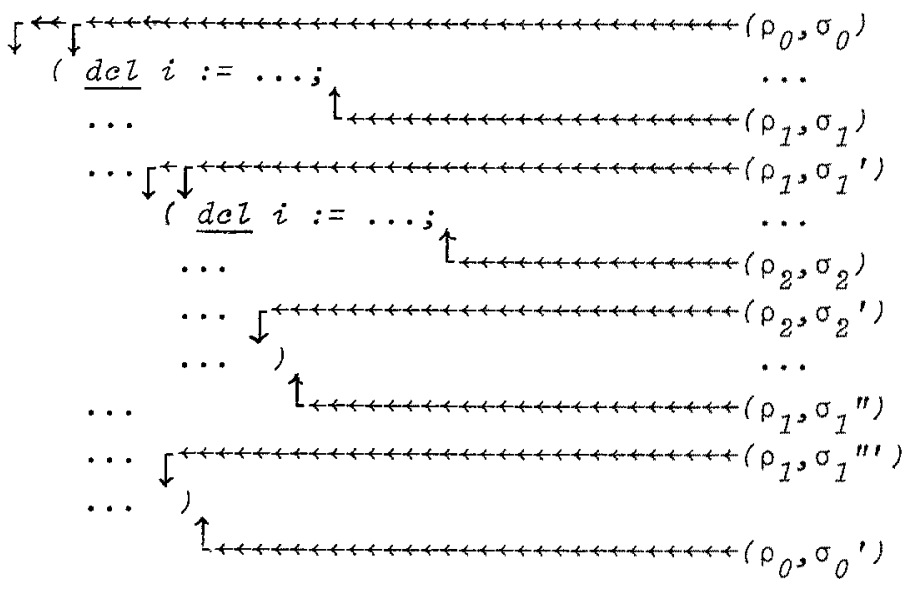

The environment immediately prior to outer block elaboration is $\rho_{0}$ and the state is $\sigma_{0}$. These are not detailed. Obeying the 'first' declaration results in the elaborator claiming a new, fresh storage cell for $i$, let us call its location $i_{i}$. Thus:

$$
\sigma_{1}=\sigma_{0} \cup\left[2 i_{1} \rightarrow o_{1}\right]
$$

with:

$$
\rho_{1}=\rho_{0}+\left[i \rightarrow 2 i_{1}\right]
$$


Thus we use $U$ as an abstraction of the storage allocation operation -$U$ since $z i_{1}$ is new, i.e. not in the domain of $\sigma_{0}: z i_{1} \sim \in$ dom $\sigma_{0}$ ! But we use + as an abstraction of the environment 'update' action -- $i$ may namely be already recorded in $\rho_{0}$. Observe that we do "fall back" to $\rho_{0}$ when "falling through" this, the outer, block. Similarly:

$$
\sigma_{1}^{\prime}=\sigma_{0}^{\prime} \cup\left[\tau_{i} \rightarrow \sigma_{1}^{\prime}\right]
$$

where the ' on $\sigma_{0}$ shall indicate that variables outside this block may have had their values updated. Also $i$ may have been updated $\left(o_{1}^{\prime}\right)$ : And:

$$
\begin{aligned}
& \rho_{2}=\rho_{1}+\left[i \rightarrow 2 i_{2}\right]=\rho_{0}+\left[i \rightarrow 2 i_{2}\right] \\
& \sigma_{2}=\sigma_{1}^{\prime} u\left[i i_{2} \rightarrow o_{2}\right]=\sigma_{0}^{\prime} u\left[z i_{1} \rightarrow o_{1}^{\prime}, i i_{2} \rightarrow o_{2}\right]
\end{aligned}
$$

That is: the storage claimed for the inner blocks' $i$ is also fresh, not clashing with the storage for the outer blocks' $i$.

Observe how environments, e.g. $\rho_{1}$, remain constant in the outer block.

$$
\begin{aligned}
& \sigma_{2}^{\prime}=\sigma_{0}^{\prime \prime} \cup\left[z i_{1} \rightarrow o_{1}^{\prime \prime}, z_{2} \rightarrow o_{2}^{\prime}\right] \\
& \sigma_{1}^{\prime \prime}=\sigma_{2}^{\prime} \backslash\left\{2 i_{2}\right\}=\sigma_{0}^{\prime \prime} \cup\left[z i_{1} \rightarrow o_{1}^{\prime \prime}\right] \\
& \sigma_{1}^{\prime \prime}=\sigma_{0}^{\prime \prime} \cup\left[z i_{1} \rightarrow o_{1}^{\prime \prime}\right] \\
& \sigma_{0}^{\prime}=\sigma_{1}^{\prime \prime}\left\{2 i_{1}\right\}=\sigma_{0}^{\prime \prime}
\end{aligned}
$$

Thus freeing, or deallocation of storage for declared variables follow the block structure, i.e.: is performed as part of the block epilogue. The MAP operator abstracting deallocation is: -

We now turn to a brief justification for the use of both environments and stores. The above example of nested blocks redefining variable names is not a sufficiently convincing example. Static renaming of e.g. the inner $i$ to e.g. $j$ would permit modeling stores as Id $\vec{m}$ OBJ. If, however, any block was [part of] a recursively defined procedure static renaming would not solve the problem of nested activations' $i$ denoting distinct locations -- which are the semantics we normally expect:

In example 31 and onwards we use $E N V$ and sometimes $\Sigma$ as follows: 


$$
\begin{array}{ll}
E N V & =I d \vec{m} L O C \\
\Sigma & =L O C \vec{m}^{O B J}
\end{array}
$$

Finally, as a last example, we show the modeling of variable referencing, value access and of assignment.

For the elaborator 'state' $(\rho, \sigma)$ elaboration of some $v \in$ dom $\rho$ is modeled ( $\Delta$ ) as:

$$
v \quad \Delta \quad \rho(v)
$$

Accessing the contents of a variable $v$ :

$$
\text { ev } \quad \Delta \quad \sigma(\rho))
$$

and assigning the object (value) 0 to variable $v$ changes the state 0 to:

$$
v:=0 \quad \Delta \quad \sigma+[\rho(v) \rightarrow 0]
$$

\section{A Last Example, $\underline{3} 1$ :}

The variables of some source language are either of scalar or array type with all array objects being scalars. Variable references, however, are to scalars only, i.e. not to arrays or array-slices thereof.

The syntactíc domaiㅡㄴ as a map from variable name identifiers to their type:

$1 \quad$ Block $\quad: \therefore \quad(I d \vec{m}$ Type $) \ldots$ Stmt*

with :

2

$$
\text { Type } \left.\quad \therefore \text { Scalar-type [Expr }{ }^{+}\right]
$$

i.e. with types having an optional [...] array upper bounds expression list, Expr ${ }^{*}$. If the optional part is absent, i.e. nil, the variable is a scalar.

The corresponding semantie domaiㅡ of storages can be modeled as a map from scalar locations to scalar values. 
where:

\begin{tabular}{|c|c|c|c|}
\hline 4 & $B 002$ & BOOL I & I undefined \\
\hline 5 & Intg & INTG & I undefined \\
\hline
\end{tabular}

Environments keep track of variable name associations:

6 $E N V=I d \vec{m}$ LOC

with :

7 LOC $\quad=$ Scalar-LoC $\mid$ Array-LoC

8 Array-Loc $=\left(N_{1}+\underset{m}{\leftrightarrow}\right.$ Int-LoC $)$ U $\left(N_{1}+\underset{m}{+}\right.$ Bool-Loc $)$

9 Sealar-Loe $=$ Int-LoC 1 Bool-Loe

with the constraint that the integer index lists of any given array location forms a 'rectangle':

$10.0 \quad(\forall z \in A r r a y-L O C)$

$.1 \quad\left(\exists i z \in N_{1}^{+}\right)(\underline{\text { dom }} z=\operatorname{rectang} z(i z))$

where we informally explain rectangle:

$11.0 \quad$ rectangle $\left(\left\langle u b_{1}, u b_{2}, \ldots, u b_{n}\right\rangle\right)=$

$.1 \quad\left\{<i_{1}, i_{2}, \ldots, i_{n}>\mid i_{k} \in\left\{1: u b_{k}\right\} \wedge 1 \leq k \leq n\right\}$.

The it 'picked' is (to be) the tuple, whose length corresponds to the array-dimension, and whose $k^{\prime}$ th element $\left(u b_{k}\right)$ is the upper-bound index for the $k^{\prime}$ th dimension, all of whose lower-bounds are 1 !

A particular storage is modeled (here) as a global meta-variable, STG:

deI STG: $:=[]$ type $S T G$

In the block prologue (.1-.2) locations of block declared variables are (automatically) allocated, and these are likewise freed $(.4-.8$ ) in the block epilogue: 
$13 \operatorname{int}-B \operatorname{Zock}(m k-B \operatorname{Lock}(\operatorname{deZs}, \ldots, s t \tau))(\rho)=$

.1

$$
\text { (Let } \rho^{\prime}: \rho+[i d \rightarrow \operatorname{get-Zoc}(\operatorname{dels}(i d))(\rho)
$$

.2

I id $\in$ dom dels ];

.3

int-Stmt-iist(sti)( $\left.\rho^{\prime}\right)$;

.4

let loes $=\left\{\rho^{\prime}(i d) \mid i d \in \underline{\text { dom }}\right.$ dels $\}$ in

.5

Let slocs $=\{l \mid(($ leloos $) \wedge$ is-Scalar-Loc $(2))$

.6

$v\left((Z \in \underline{x n g} a Z) \wedge\left(a L \in Z_{0 c s}\right) \wedge\right.$

.7

is-Array-Loc(al))\} in

.8 STG $:=$ eSIG $($ stoes

with:

$14.0 \quad g e t-l o c(m k-T y p e(s c t p, b d \tau))(\rho)=$

.1

if $b d z=\underline{n i l}$

.2

then

.3

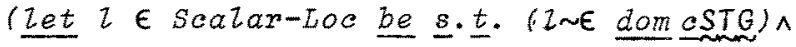

.4

.5

.6

STG := cSIG $\cup$ [l $\rightarrow$ undefined $]$;

Z-tp-match $(\operatorname{setp}, \tau)$;

return (2))

.7

else

.8

.9

.10

.11

.12

.13

.14

.15

.16

.17

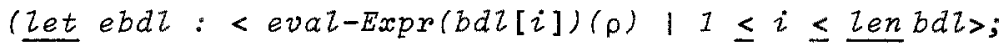

if $(\exists i \in\{1: \underline{\text { Zen }} e b d z\})(e b d z[i]<1)$

then error

else

let $l \in$ Array-Loc be s.t.

( (scl $\in$ rng $Z)>l-t p-\operatorname{match}(\operatorname{set} p, s c l))$

$\wedge($ dom $Z=$ rectangle $(e b d L)$

$\wedge(\underline{r n g} Z \mathrm{n}$ dom e STG $)=\{\})$;

STG $:=$ eSTG U [ sol $\rightarrow$ undefined $\mid$ sel $\in$ rng $Z$ ]; return(2))

and

$15.0 \quad z-t p-m a t a h(t p, z)=((t p=\underline{B} \underline{O L L}) \rightarrow i s-B \circ o z-L O C(z)$,

$(t p=I N T) \quad \rightarrow i s-I n t-L o c(Z))$

Assuming that the state, $\Sigma$, is describable as:

16

$$
\Sigma=\quad \operatorname{STG} \underset{m}{m} S T G
$$

(i.e. $\Sigma$ is a one-point (STG) map), the type of the above functions are: 


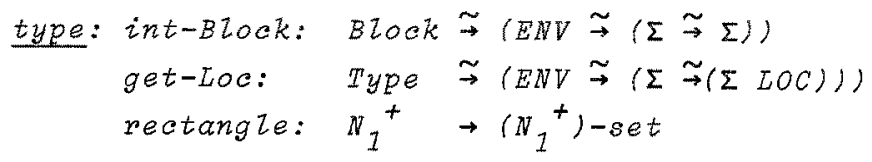

\section{TREES}

\section{Examples $\underline{32}-\underline{3} \underline{3}:$}

Blocks of some source language consists of three distinct parts: an unordered set of variable declarations, an unordered set of distinctly named procedures and an ordered sequence of one or more statements.

$$
\left(\text { Var-set Id } \vec{m} \text { Proc } S t m t^{+}\right)
$$

The above is a domain expressions. If Var denotes the domain of variable identifiers, Id that of procedure identifiers, Proc the domain of procedures and Stmt the domain of statements; then the above domain expression abstracts a nameless domain of blocks. These are tre통, whose first subcomponents are sets of variable names, second subcomponents are maps from identifiers to procedures, and whose last subcomponents are tuples of statements.

If suitably decorated $v^{\prime} s, i d^{\prime} s, p^{\prime} s$ and $s^{\prime} s$ stand for variables, procedure names, procedures, and statements, then:

$$
m k\left(\left\{v_{1}, v_{2}, \ldots, v_{v}\right\},\left[i a_{1} \rightarrow p_{1}, i a_{2} \rightarrow p_{2}, \ldots, i a_{p} \rightarrow p_{p}\right],<s_{1}, s_{2}, \ldots, s_{s}>\right)
$$

and:

$$
\left(\left\{v_{1}, v_{2}, \ldots, v_{v}\right\},\left[i a_{1} \rightarrow p_{1}, i a_{2} \rightarrow p_{2}, \ldots, i a_{p} \rightarrow p_{p}\right],<s_{1}, s_{2}, \ldots, s_{s}>\right)
$$

(the latter constructor expression having dropped the nameless constructor function, $m k$ ) represent the way we write down such composite objects. If $b$ is a block, or more precisely, if $b$ is an object in the domain specified above, then the following meta-language combinators:

$$
\begin{aligned}
& \text { Zet } m k(v s, p m, s t)=b_{3} \\
& \text { Zet }(v s, p m, s z)=b ;
\end{aligned}
$$


provide means of decomposing nameless tree structured objects, here modelling blocks, into their proper constituents -- here the set of variables, $v s$; the map from procedure names to procedures, $p m$; and the statement list, sl.

The domain expression:

$$
\text { (s-vars:Var-set s-procm: (Id } \left.\vec{m} \text { Proc) s-stmtl:Stmt }{ }^{+}\right)
$$

(with inner (,)'s, around $I d \vec{m}$ Proc, used only as syntactic delimiter) defines the same domain as above, but in addition defines three selector functions, here arbitrarily named: s-vars, s-procm, s-stmtz. Now:

$$
\text { Let } \begin{aligned}
v s & =s-\operatorname{vars}(b), \\
p m & =s-\operatorname{procm}(b), \\
s z & =s-s \operatorname{tmt} z(b) ;
\end{aligned}
$$

has the same effect as the previous decomposition combinators. In other words, if $v \varepsilon \in V a r-s e t, p m \in I d \vec{m}$ proc, $s l \in S t m t^{*}$, then:

$$
\begin{aligned}
& s-v a r s(m k(v s, p m, s z))=v s, \\
& s-p \operatorname{rocm}(m k(v s, p m, s Z))=p m, \\
& s-s t m t Z(m k(v s, p m, s Z))=s Z .
\end{aligned}
$$

We can give a name to the above block domain, e.g. Block:

$$
\text { Block } \quad \therefore \text { Var-set Id } \vec{m} \text { Proe stmt }
$$

-- note the dropping of $(,)^{\prime} s$ : With $v s, p m$ and $s l$ as before:

$$
m k-B \text { Zock (vs, pm, sl) }
$$

would now have to be our way of writing down instances of blocks. The trees denoted by Block are now named (Block). Also here we could introduce selectors: 
Block :: s-vars:Var-set s-procm:(Id $\vec{m}$ Proc) s-stmt stmt $^{+}$

(where the (,)'s, around Id $\vec{m}$ Proc, again are used as syntactic delimiters). And again:

$$
s-\operatorname{vars}(m k-B \text { Lock }(v s, \ldots, \ldots))=v s
$$

etc.. A benefit derived from naming the class of constructed tree objects, here Block, is that any object can be tested for membership of the named domain:

$$
\text { is-Brock }(b)
$$

is true provided $b$ is the result of some $m k-B$ lock $(v s, p m, s l)$-- for any applicable $v s, p m$ and $s i$.

\subsection{Defining Domains of TREE Objects}

Two means of defining domains of tree objects exist in the metalanguage:

(1) Named: Using the abstract syntax rule definition symbol $\therefore$ to separate definiens from definiendum:

$$
D \quad \therefore D_{1} D_{2} \cdots D_{n}
$$

(2) Anonymous: Using the domain expressions operator (...),

$$
\left(D_{1} D_{2} \cdots D_{n}\right)
$$

The former defines named or root labelled trees:

$$
\left\{m k-D\left(d_{1}, d_{2}, \ldots, d_{n}\right) \mid i s-D_{i}\left(d_{i}\right) \text { for all } i\right\}
$$

The latter defines unnamed trees:

$$
\left\{m k\left(d_{1}, d_{2}, \ldots, d_{n}\right) \mid i s-D_{i}\left(d_{i}\right) \text { for } a 11 i\right\}
$$

or, dropping the $m k$ : 


$$
\left\{\left(d_{1}, d_{2}, \ldots, d_{n}\right) \mid \text { is }-D_{i}\left(d_{i}\right) \text { for alz } i\right\}
$$

\section{Example 3 느:}

Statements of a source language are either assignment statements, while-do statements or if-then statements:

$$
\begin{aligned}
& \text { Stmt }=\text { Asgn | While | IfThen } \\
& \text { Asgn }:: \text { Id Expr } \\
& \text { While }:: \text { Expr Stmt } \\
& \text { IfThen : Expr Stmt }
\end{aligned}
$$

Observe how the two last domains:

$$
\begin{aligned}
& \{m k-W h i z e(e, s) \mid i s-\operatorname{Expr}(e) \wedge i s-S t m t(s)\} \\
& \{m k-I f T h e n(e, s) \mid i s-E x p r(e) \wedge i s-S t m t(s)\}
\end{aligned}
$$

are disjoint -- simply because the names of their object make func tions, $m k-$ While and $m k-I f T h e n$, are distinct:

\subsubsection{Tree Constructor Axioms}

For any two $D^{\prime}$ and $D^{\prime \prime}$ identifiers being definienses of tree constructing abstract syntax rules:

$$
\begin{array}{llllll}
D^{\prime} & \therefore & A_{1} & A_{2} & \cdots & A_{m} \\
D^{\prime \prime} & \therefore & B_{1} & B_{2} & \cdots & B_{n}
\end{array}
$$

objects :

$$
\begin{aligned}
& m k-D^{\prime}\left(a_{1}, a_{2}, \ldots, a_{m}\right), \\
& m k-D^{\prime \prime}\left(b_{1}, b_{2}, \ldots, b_{n}\right)
\end{aligned}
$$

are identical if and only if (iff): 


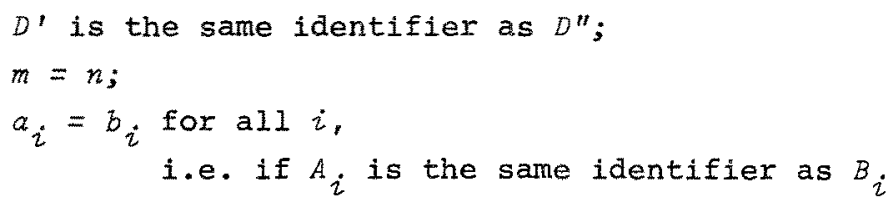

that is, iff the two rules above are the same.

For any two implicit tree domain denoting expressions:

$$
\left(\begin{array}{cccc}
A_{1} & A_{2} & \cdots & A_{m}
\end{array}\right)
$$

objects :

$$
\begin{aligned}
& m k\left(a_{1}, a_{2}, \ldots, a_{m}\right) \\
& m k\left(b_{1}, b_{2}, \ldots, b_{n}\right)
\end{aligned}
$$

or, which is the same:

$$
\begin{aligned}
& \left(a_{1}, a_{2}, \ldots, a_{m}\right) \\
& \left(b_{1}, b_{2}, \ldots, b_{n}\right)
\end{aligned}
$$

are identical iff:

$$
\begin{aligned}
& m=n, a_{i}=b_{i} \text { and } \\
& A_{i} \text { is the same identifier as } B_{i} \text { for all } i .
\end{aligned}
$$

\section{Example 3 므-3 6 :}

The abstract syntax rules:

$$
\begin{aligned}
& \text { CrLG } \quad=\text { Fid } \vec{m}(K t p D t p) \\
& \text { Define } \quad \therefore \text { Fid (Ktp Dtp) }
\end{aligned}
$$

are intended to define the semantic domain of file description calatoGues, respectively the syntactic domain of file definition 
commands. To each file, identified by its name (in Fid) is associated, in the catalogue, its description -- namely a 'pair' consisting of a Key type-, and a Data type- indication, i.e, an object in (Ktp Dtp). A file definition command names, say fid, the file to be defined ( $f i d \in E i d$ ), and gives the Key type-, and Data type indication, i.e. an object likewise in (Ktp Dtp). In particular a catalog, etzg, may look like:

$$
\begin{aligned}
& {\left[f i d_{1} \rightarrow m k\left(k t p_{1}, d t p_{1}\right),\right.} \\
& \quad \ldots \\
& \left.f i d_{n} \rightarrow m k\left(k t p_{n}, d t p_{n}\right)\right],
\end{aligned}
$$

with a define command looking like:

$$
m k-D e f i n e(f i d, m k(k t p, d t p))
$$

Dropping the somewhat superfluous mentioning of the otherwise nameless $m k$ (prefixing $(. .$.$) ), we get that ctig, upon execution of the$ define command, is augmented to:

$$
\operatorname{ctig} \cup[f i d \rightarrow(k t p, d t p)]
$$

provided, of course fid $\sim \in$ dom ctzg:

As another example of nameless tree constructions let us abstract the syntactic domain of procedures of some source language:

$$
\text { Proc }: \therefore(I d T p)^{*} B \text { rock }
$$

The form (Id Tp)* could be written Parm*, i.e.:

$$
\text { Proc :: Parm* Block }
$$

provided:

$$
\text { Parm :: Id Tp }
$$

In the former case the parameter list is abstracted as a possibly 
zero-length tuple of parameters, these being abstracted as nameless trees, themselves being 'pairs' of formal parameter Identifiers and their Types (not being further specified). In the latter case these parameters are abstracted as named (Parm) trees. Given that suitably decorated $i d^{\prime} s, t p ' s$ and $b^{\prime} s$ denote objects in $I d, T p$ and Block, respectively, we get, in the two cases, that:

$$
m k-\operatorname{Proc}\left(\left\langle\left(i d_{1}, t p_{1}\right),\left(i d_{2}, t p_{2}\right), \ldots,\left(i d_{n}, t p_{n}\right)\right\rangle, b\right)
$$

respectively:

$$
m k-\operatorname{Proc}\left(\left\langle m k-\operatorname{Parm}\left(i d_{1}, t p_{1}\right), \ldots, m k-\operatorname{Parm}\left(i d_{n}, t p_{n}\right)>, b\right)\right.
$$

display instances of procedures according to either abstraction. Thus observe that the (,)'s in (Id $T p)^{*}$ serve the double function of constructing a domain of anonymous trees, as well as indicating the scope of the tuple domain building * operator.

\subsection{Representing Instances of TREE Objects}

Given objects $o_{1}, o_{2}, \ldots, o_{n}$ of not necessarily distinct domains, e.g.: $D_{1}, D_{2}, \ldots, D_{n}$, the constructor expressions:

$$
\left(o_{1}, o_{2}, \ldots, o_{n}\right), m k\left(o_{1}, o_{2}, \ldots o_{n}\right)
$$

denote (identical) tree objects of domain:

$$
\left(D_{1} D_{2} \ldots D_{n}\right)
$$

i.e. an anonymous tree. Given that there exists an abstract syntax rule of the form:

$$
D \quad: \quad D_{1} D_{2} \cdots D_{n}
$$

the constructor expression:

$$
m k-D\left(o_{1}, o_{2}, \ldots, o_{n}\right)
$$

denotes a tree object of domain $D$, 1.e. a root labelled tree. 


\subsection{TREE Operations: Selector Functions}

The only special operation defined on TREEs is the selector function. Names of selector functions are either explicitly, or implicitly, definable.

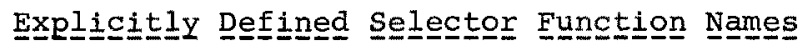

In the named tree constructing abstract syntax rule:

$$
D \quad: \quad s-n m_{1}: D_{1} s-n m_{2}: D_{2} \cdots s-n m_{2}: D_{2}
$$

as well as in the anonymous tree constructing domain expression:

$$
\left(s-n m_{1}: D_{1} s-n m_{2}: D_{2} \cdots s-n m_{2}: D_{2}\right)
$$

the identifiers:

$$
s-n m_{1}=s-n m_{2}, \ldots, s-n m_{2}
$$

denote selector functions obeying:

$$
\begin{aligned}
& s-n m_{2}\left(m k-D\left(o_{1}, o_{2}, \ldots, o_{l}\right)\right)=o_{1} \\
& s-n m_{2}\left(m k-D\left(o_{1}, o_{2}, \ldots, o_{l}\right)\right)=o_{2} \\
& \cdots \\
& s-n m_{2}\left(m k-D\left(o_{1}, o_{2}, \ldots, o_{2}\right)\right)=o_{l}
\end{aligned}
$$

respectively

$$
\begin{aligned}
& s-n m_{1}\left(\left(o_{1}, o_{2}, \ldots, o_{2}\right)=o_{1}\right. \\
& s-n m_{2}\left(\left(o_{1}, o_{2}, \ldots, o_{2}\right)\right)=o_{2} \\
& \cdots \\
& s-n m_{l}\left(\left(o_{1}, o_{2}, \ldots, o_{2}\right)\right)=o_{2}
\end{aligned}
$$

for all $o_{1}, o_{2}, \ldots, o_{2}$.

In the above, explicit, selector function name defining forms, it is assumed that all identifiers $s-n m_{i}$ and $s-n m_{j}$ are distinct. You may wish to omit any subset of these, including all, as we have done in the past. 


\section{Impliiㅁ느니 Defined Selector Function Names}

Assuming uniqueness of $D_{i}$, for some or all $i$ in $\{1: 2\}$ in:

$$
D \quad: \quad D_{1} D_{2} \cdots D_{2}
$$

respectively:

$$
\left(D_{1} D_{2} \cdots D_{2}\right)
$$

and in particular, assuming that $D_{i}$ is an identifier, the above two tree domain constructing forms define the selector function:

$$
s-D_{i}
$$

\section{Example 37 :}

In the source language statement syntax rules:

$$
\begin{aligned}
& \text { Stmt }=\text { Asgn I While } \\
& \text { Asgn } \therefore \text { Id Expr } \\
& \text { WhiZe } \therefore \text { Expr Stmt }
\end{aligned}
$$

s-Id applies to Asgn objects and yields the Id component; s-Expr applies to Asgn and While objects and yield the Expr component; and 8 -Stmt applies to whize objects and yield the proper Stmt component. We emphasize: 'proper component' since any Whize object, by the first abstract syntax rule, is itself a stmt object.

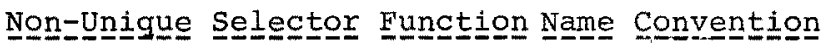

For the common case where two or more identifiers, $D_{i}$ and $D_{j}$, of either:

$$
D \quad \therefore \quad D_{1} D_{2} \cdots D_{2}
$$

or:

$$
\left(D_{1} D_{2} \cdots D_{2}\right)
$$

are the same, the following convention is adopted: 
In the form $D_{1} D_{2} \cdots D_{2}$ let there be $k$ distinct occurrences of the same identifier, say $C$, then:

$$
s-C-1, s-C-2, \ldots, s-C-k
$$

are the selector functions which select the 1st, the 2 nd,..., respectively the $k$ th proper $C$ component -- from left-to-right.

\section{Example 38 :}

In the source language statement syntax rules:

$$
\begin{aligned}
& \text { Stmt }=\ldots \text { | Eor | If } \\
& \text { For } \quad \therefore \text { Id Expr Expr Expr Stmt* } \\
& \text { If } \quad \therefore \text { Expr Stmt Stmt }
\end{aligned}
$$

the selector function $s-E x p r-1, s-E x p r-2$ and $s-E x p r-3$ select the same For object components as would s-init, s-step and s-iimit in:

$$
\text { For :: Id s-init:Expr s-step:Expr s-iimit:Expr Stmt*; }
$$

and $s-S t m t-1$ and $s-S t m t-2$ selects the same If object components as would s-then and $s-e$ se in:

$$
\text { If } \quad: \text { Expr s-then:Stmt s-else:Stmt. }
$$

\section{Programming Notetes}

The meta-language leaves unexplained the names of the implicitly defined selector functions in such forms as:

and:

$$
\text { Block :: Id-set }\left(I d \vec{m}^{\text {Pre }}\right) \text { Stmt* }
$$

$$
\text { For } \quad \therefore \quad s-c v: I d(s-i: E x p r s-b: \operatorname{Exp} s-t: E x p r)^{+} s t m t^{*}
$$

In the latter form there are only two implicitly defined selector functions, namely the two selecting the (Expr Expr Expr) ${ }^{*}$ and Stmt tuple objects of For objects.

The reason for introducing explicit selector function names is mostly pragmatic. That is: there is, in most cases, no technical need for in- 
venting names. To see this, recall that the technical purpose of selec. tor functions is to select proper (sub-) components of trees. But this could as well be done by a 'reverse' use of the $m k$ function! Let $e . g$. $t$ be an object of

$$
\left(D_{1} D_{2} \cdots D_{n}\right)
$$

then: the let clause:

$$
\text { Let }\left(o_{1}, o_{2}, \ldots, o_{n}\right)=t
$$

so-to-speak decomposes $t$ into its $n$ subcomponents. So would:

$$
\text { Let } \begin{aligned}
o_{1} & =s-D_{1}(t), \\
o_{2} & =s-D_{2}(t), \\
\ldots & =s-D_{n}(t) \\
o_{n} &
\end{aligned}
$$

provided, of course, all $D_{i}$ were distinct (and) identifiers! similar for $D$ objects $t$, where:

$$
D \quad: \quad D_{1} D_{2} \ldots D_{n}
$$

leading to:

Let $m k-D\left(o_{1}, o_{2}, \ldots, o_{n}\right)=t$

etc. . 


\section{EUNCTIONS}

By a function, we shall -- in a somewhat circular fashion -- understand an operation which, when applied to something, which we shall call its argument yields a certain thing as the value of the function for that argument.

The object to which the function is applicable, i.e. for which it is guaranteed to yield a value (defined result), constitutes the domain of the function. The yielded values constitute the range for: codomain) of the function.

Two functions are identical iff they (1) have the same domain, (2) the same range, and (3) for each argument in the domain the same value. FunCTion equality is, however, not a defined operation.

To denote the value of a function for a given argument we write a name of the function, say $f$, followed by a name of the argument, say $a$; the latter, the former or both possibly enclosed between parentheses:

$$
f a, f(a),(f)(a),(f a),(f) a
$$

Many functions can more easily be described algorithmically, i.e. by a recipe for how to compute the result value given an argument value, than by explicit or implicit enumeration. Moreover, some, if not most such, functions, which we wish to manipulate (create, pass and apply), have an infinite domain -- and by the pragmatics of MAPs could not be constructed as such. Finally: many functions can best be described in an implicit, or even recursive, way, which certainly does not conjure the image, or thought, of its graph being computed at the time of definitions. (By the graph of a function we understand the set of all argument result 'pairs'.)

For maps this graph is indeed being computed at "time" of definition, whereas we may think of this graph never being computed in connection with the kind of function definitions we are interested in in this section. 
Examples 39-40:

The following is a block-expression of the meta-language; its value is that of the denotation of $f$, which is the factorial function:

$$
\text { (Let } f(n)=\text { if } n=0 \text { then } 1 \text { else } n \times f(n-1) \text { in }
$$

Let, as another example, the procedure header -- exclusive of procedure name -- and procedure body, of some source language be abstract syntactically describable as:

$$
\text { Pre :: Id* Block }
$$

Here Prc is the name of the domain of procedure definienses (header + body, - name), Id of the domain of formal parameter names, and Block of blocks -- as e.g. statement blocks.

The meaning of a procedure, i.e. the denotation of a procedure name, may, according to the school of mathematical semantics, be taken as a function from argument lists to the denotation of blocks. If the denotation of these latter are functions from states $(\Sigma)$ to states then:

$$
\text { Pre: } A R G^{*} \stackrel{\rightarrow}{\rightarrow}(\Sigma \simeq \Sigma)
$$

Given a Pro object, pro, i.e. one of the form $m k-P r c(i d l, b l)$, we now give the function which itself yields the denotation of pre. Let, as a last preparation, procedure denotations be functions of the defining rather than calling environment.

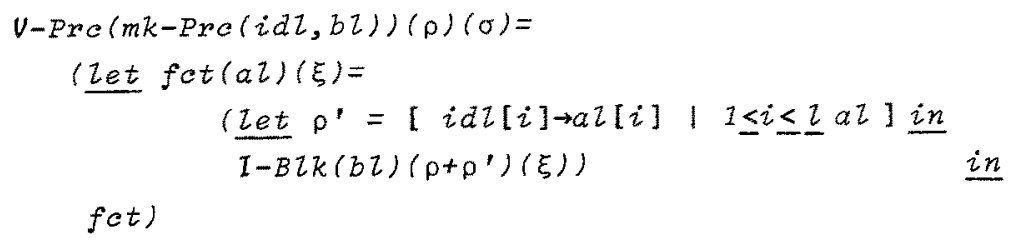

Observe the following: evaluation of a Procedure denotation takes place in a defining environment, $\rho$, and state, $\sigma$. The state is ignored, and need thus not have been shown. The result of Procedure 
eValuation is a function, fet. This function is completely described in the three-line let clause. The definition given there can be read as follows: fet is that function which when applied to some argument list, $a \eta$, and in some state, $\xi$, will yield a new state. This new state results from Interpreting the $B$ lock $b$ in the defining environment ('slightly') extended -- by the bindings of formal parameter identifiers, $i d z[i]$, to actual, call time, arguments, $a z[i]$-- and in the calling state, $\xi$. We do not here display the $I-B$ Zock elaboration function, but see section 6.3 .

\subsection{Defining Domains of EunCTion Objects}

Let $A$ and $B$ denote Domains. To define the class of implicitly $\lambda$-defined objects which are partial, respectively total functions from $A$ into $B$, we use the domain expression operators $\stackrel{\sim}{\rightarrow}$, respectively $\rightarrow$ :

$$
A \stackrel{\sim}{\sim}, \quad A \rightarrow B \quad
$$

\section{Example 4I:}

Applying the ability to describe function spaces to the built-in operations of the meta-language itself, we can now list the logical type of these:

\begin{tabular}{|c|c|c|c|c|c|c|}
\hline \multirow[t]{9}{*}{ Sets: } & \multirow{9}{*}{ type: } & U: & $S E T$ & $S E T$ & $\rightarrow$ & $S E T$ \\
\hline & & $n:$ & $S E T$ & $S E^{\prime} T$ & $\rightarrow$ & $S E T$ \\
\hline & & -: & $S E T$ & $S E T$ & $\rightarrow$ & $S E T$ \\
\hline & & ㄷ: & $S E T$ & $S E T$ & $\rightarrow$ & BOOL \\
\hline & & $c:$ & $S E T$ & $S E T$ & $\rightarrow$ & BOOL \\
\hline & & power: & & $S E T$ & $\rightarrow$ & $S E T$ \\
\hline & & union: & & $S E T$ & $\dddot{\rightrightarrows}$ & $S E T$ \\
\hline & & $\epsilon$ & $O B J$ & $S E^{2} T$ & $\rightarrow$ & BOOL \\
\hline & & card & & $S E T$ & $\rightarrow$ & $N_{0}$ \\
\hline
\end{tabular}




\begin{tabular}{|c|c|c|c|c|}
\hline Tuples: type: & *: & TUPLE & TUPLE & $\rightarrow T U P L E$ \\
\hline & $\underline{h}, \underline{h d}:$ & & TUPLE & $\widetilde{O O B J}$ \\
\hline & $t, t z:$ & & TUPLE & $\stackrel{T}{\rightarrow} U P L E$ \\
\hline & I, Zen: & & TUPLE & $\rightarrow n_{0}$ \\
\hline & {$[]:$.} & TUPLE & $N_{1}$ & $\stackrel{O}{O} O B J$ \\
\hline & elems: & & TUPLE & $\rightarrow S E T$ \\
\hline & ina: & & TUPLE & $\rightarrow N_{1}-s e t$ \\
\hline & cone: & & TUPLE & $\stackrel{\sim}{\rightarrow}$ TUPLE \\
\hline & $+:$ & TUPLE & $M A P$ & $\sim T U P L E$ \\
\hline & $\therefore$ & TUPLE & $N_{1}-s e t$ & $\stackrel{\sim}{\rightarrow} U P L E$ \\
\hline
\end{tabular}

\begin{tabular}{|c|c|c|c|c|c|}
\hline \multirow[t]{8}{*}{ Maps: } & \multirow[t]{8}{*}{ type: } & U: & $M A P$ & $M A P$ & $\underset{\rightarrow}{\sim} M A P$ \\
\hline & & $+:$ & $M A P$ & $M A P$ & $\rightarrow M A P$ \\
\hline & & $-:$ & $M A P$ & $S E T$ & $\rightarrow M A P$ \\
\hline & & 1: & $M A P$ & $S E T$ & $\rightarrow M A P$ \\
\hline & & (.) : & $M A P$ & $O B J$ & $\stackrel{\sim}{\sim} O B J$ \\
\hline & & dom: & & MAP & $\rightarrow S E T$ \\
\hline & & rng: & & MAP & $\rightarrow S E T$ \\
\hline & & $\because$ & $M A P$ & MAP & $\stackrel{\sim}{\rightarrow} M A P$ \\
\hline
\end{tabular}

The reason why certain of these operations are partial functions will now be explained. The union operation applies to sets of sets, SET just expresses: sets of objects. We could get around this by writing SET-set whereby: type: union: SET-set $\rightarrow$ SET, i.e. union is total over SET-set. hd and tz applies to non-zero length tuples, i.e.: type: ha: $O B J^{+} \rightarrow O B J, \underline{t z}: O_{B J} \rightarrow O B J^{*}--$ note totality. Indexing, $[i]$, must have $i$ lie in the index set of the tuple, otherwise undefined. cone is to tuples what union is to sets, i.e. type: cone: TUPLE* $\rightarrow$ TUPLE. Updating $\left(^{(}\right)$tuple elements must have MAP in $\left(N_{1} \vec{m}\right.$ OBJ) with domain elements be in index set of tuple. So not even: type: $+:$ TUPLE $\left(N_{1} \vec{m}\right.$ OBJ $) \stackrel{\sim}{\rightarrow}$ TUPLE would make + total over defined domain.

\subsection{Representing Instances of FunCTion objects}

This section is somewhat differently organized than were otherwise comparable earlier sections ( $i .2$ for $i=2,3,4,5)$. In a number of subsec tions we recount basic aspects of so-called $\lambda$-expressions. 
We shall shortly, in a sequence of eight small steps, arrive at an understanding of functional abstraction. The idea being to write forms which denote functions. One such class of forms were the map expressions covered in section 4. Another such form is that of $\lambda-$ expressions. Map expressions are used to define functions whose argument-value association is known (i.e. being fixed) at the instance of definition, and whose domain-range sets are individually known and finite.

\section{Functional Abstraction}

(0) There are simple and composite proper names.

(1) Simple names are either arbittrari thing, or their denotation has been assigned an arbitrary name. Composite names express through their structure some analysis of the way in which they denote.

(2) A gonstant is a proper name having a fixed, or single denotation.

(3) A variable is a proper name whose denotation may range over a set of valüus.

(4) An expressíngn is a name containing other names as proper constituents.

Composite names are expressions.

(5) A form is either a variable or an expression in which one or more proper names have been replaced by variables.

(6) In order to speak about the function of a free variable that a form is we abstract the form by prefixing it with a sequence of three symbols: a $\lambda$, the free variable and a dot.

$\lambda y \cdot 3+y$ is the function of $y$ that $3+y$ is; i.e. which increments any number by three.

(7) The passage from a form to an associated function is called

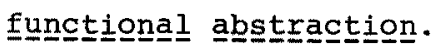


Functionally abstracting the forms: $3+y, x+y$ and $\lambda y \cdot x$ into e.g. $\lambda y \cdot z+y, \lambda x \cdot \lambda y \cdot x+y$ respectively $\lambda x \cdot \lambda y \cdot x$ results in the incrementby-3 function, the addition (of two numbers) function and the function from (say) integers $(x)$ to constant functions from (say) booleans $(y)$ to that integer $x$.

In the above we have employed a rather restricted and not completely detailed use of the concepts: names, expressions and forms. The term. expression shall imply the inclusion of simple constants and variable (identifier)s. Forms are henceforth expressions.

\section{$\lambda$-expressions}

Any clause (expression or statement), clause, of the meta-language can be functionally abstracted, whether containing free variables or not (for free/bound variables, see below).

Functional abstraction in zero variables is written:

$$
\lambda() . \text { clause }
$$

Writing, in the meta-language, the definition:

$$
\text { Let } f=\lambda() \text {. clause }
$$

or :

$$
\text { Let } f()=\text { clause }
$$

-- which is equivalent -- thus identifies $f$ as a name for the function of no variables that clause is. Any subsequent occurrence in the scope of the definition, i.e, use in contrast to definition, of $f$ without () shall then denote the function, whereas any subsequent occurrence in the scope of the definition of $f$ of $f()$ shall denote the elaboration of clause.

If the valuation of clause is dependent on a state -- not shown -then repeated occurrences of $f()$, elaborated in different states, may result in distinct $f(), 1 . e$. clause, 'values'. These remarks 
also apply to functions of several variables

Functional abstraction in two or more variables is written either:

$$
\lambda i d_{1} \cdot \lambda i d_{2} \cdot \ldots \cdot \lambda i d_{n} \cdot \text { clause }
$$

or:

$$
\lambda\left(i d_{1}, i d_{2}, \ldots, i d_{n}\right) . \text { clause }
$$

where $n \geq 2$, all id's distinct and ... is a shortening ellipsis extraneous to the meta-language. Let $i d_{i}$ range over $D_{i}$ and the values of (for suitable substitutions of actual for formal parameters, see below) over $D$, then the denoted function is in the space:

$$
D_{1} \rightarrow\left(D_{2} \rightarrow\left(\ldots \rightarrow\left(D_{n} \rightarrow D\right) \ldots\right)\right.
$$

respectively:

$$
D_{1} D_{2} \cdots D_{n} \rightarrow D
$$

The former form corresponds to a so-called "currying" or "schönfinckeling" of the more familiar, latter, form. The former form also permits the application of (strictly) less than, as here, $n$ arguments, namely the first (from left-to-right, say) $k(k<n)$ arguments.

Such an application then denotes a function in the space:

$$
D_{k+1} \rightarrow\left(D_{k+2} \rightarrow\left(\ldots \rightarrow\left(D_{n} \rightarrow D\right) \ldots\right)\right.
$$

where $k+1 \leq n$. [Of course, if $D$ is a itself a functional space, say $D^{\prime} \rightarrow D^{\prime \prime}$, then application of $n$ arguments will also yield a function.]

$m$-ary functions, for $m \geq 0$, can be identified:

$$
\text { Let } f\left(i d_{1}, i d_{2}, \ldots, i d_{m}\right)=\text { clause }
$$

or:

$$
\text { Let } f=\lambda\left(i d_{1}, i d_{2}, \ldots, i d_{m}\right) \text {. clause }
$$


where no two $i d_{i}$ and $i d_{j}$ are the same. If $f$ occurs free (see below) in clause then the denoted function is the so-called minimal fixpoint solution to the (recursive) equation:

$$
f\left(i d_{1}, i d_{2}, \ldots, i d_{m}\right)=\text { clause }
$$

etc. The minimal fix-point finding operation, $Y$, will be explained subsequently. We could have indicated any such intended recursion by instead defining $f$ by:

$$
\text { Letrec } f\left(i d_{1}, i d_{2}, \ldots, i d_{m}\right)=\text { elause }
$$

respectively:

$$
\text { let } f=\gamma \lambda g \cdot \lambda\left(i d_{1}, i d_{2}, \ldots, i d_{m}\right) \cdot \text { clause }^{\prime}
$$

where clause' arises from clause by substituting all free occurrences in clause of $f$ by $g$.

[Using $g$ instead of $f$ on the right-hand side is strictly speaking not necessary, since -- under the letrec versus let convention -- the left hand $f$ is not bound to any of the right-hand $f^{\prime} \mathrm{s} .1$

We shall, however, always force the user of the meta-language to mean recursion when using the defined function identifier in clause. Hence we choose to omit the rec suffix on let's and never to use the $\mathrm{Y}$ recursion maker. Consequently the only style in which a function can be recursively defined (inside a conventional function definition, see section 5) is by means of a let definition which also gives name to the function, a name whose denotation is known within the scope of the meta-language block in which the let occurred. This is in contrast to the form :

$$
\gamma \lambda f \cdot \lambda\left(i d_{1}, i d_{2}, \ldots, i d_{m}\right) \cdot \text { clause }
$$

which otherwise defines the same function, but does not further identify it: 
Free and Bound Variables, Scope

For the purpose of the definition of the concepts of free and bound variables we consider the meta-language programs (or: function definitions) as made up from the following three expression constructs:
(1) Variables
(2) Function Applications
(3) Function Abstractions

(1) $x$ is bree in $x$.

(2) $x$ is bree in $f(a)$ if it is bree in either $f$, or $a$, or both.

(3) $x$ is gree in $\lambda y$.clause if $x \neq y$ (i.e. if $x$ is an identifier distinct from $y$ ) and $x$ is free in clause.

(4) Since the non-recursive let definition block:

$$
\frac{(\text { Let }}{\text { se) }} y=\operatorname{exph} \text { in }
$$

where se is either a meta-language statement or expression, can be understood to be a syntactic sugaring of:

$$
(\lambda y \cdot s e)(\exp r)
$$

provided calleby-value is used, we see that freeness of $x$ in a let definition block follows the rules for freeness of $x$ in an application.

(5) Similar for statement composition:

$$
\begin{aligned}
& (s t m t 1 \\
& s t m t 2)
\end{aligned}
$$

only makes sense if stmt and stmt2 both denote functions from states, $\sigma$, to states, $\sigma^{\prime}$, (stores to stores), hence:

$$
(s t m t 1 ; s t m t 2) \sigma
$$

is really to be understood as: 


$$
\begin{aligned}
& \left(\underline{\text { Zet }} \sigma^{\prime}=\operatorname{stm} t 1\left(\sigma^{\prime}\right) \underline{i n}\right. \\
& \left.\operatorname{stm} t 2\left(\sigma^{\prime}\right)\right)
\end{aligned}
$$

which then reduces to:

$$
\left.\left(\lambda \sigma^{\prime} \cdot\left(\operatorname{stm} t 2\left(\sigma^{\prime}\right)\right)\right)(\operatorname{stm} t)(\sigma)\right)
$$

A variable is bound in some expression if it occurs in the expression but is not free.

It follows then that the formal parameter variables (identifiers) bind all of the free occurrences of such identifiers in the body, i.e. clause, of a $\lambda$-expression:

$$
\lambda\left(i d_{1}, i d_{2}, \ldots, i d_{m}\right) . \text { clause }
$$

The $i d_{i}$ 's are called bound varriablies .

The scope of a bound variable is the entire body-part of the $\lambda$-expression to which it belongs, with the specific exception of each properly contained $\lambda$-expression of the body-part having that same bound variable identifier.

\section{Application}

Applying a $\lambda$-defined function:

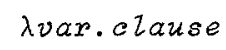

to an argument, arg:

$$
(\lambda v a r . c \text { lause) (arg) }
$$

then means to evaluate the expression clause' which arises from clause by subsstitututing all free occurrences of (the syntactic object) the identifier var in clause by the (semantic) object denoted by arg.

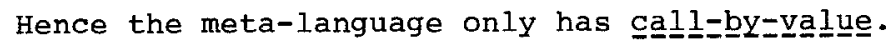

The above application rule extends to functions of several arguments. 


\section{Definition versus Application}

To write:

$$
\frac{\text { Let }}{c(f)} f(a)=\text { clause } \underline{\text { in }}
$$

means: let $f$ denote the function which satisfies the above equation and evaluate or interpret $\mathcal{C}(f)$ in such an environment where $f$ is bound to that function. It does, e.g. , not mean: evaluate clause before proceding to elaboration of $c(f)$. clause is evaluated iff $f$ is applied and then the suitable substituted clause' is evaluated everytime, afresh, whenever $f$ is so applied:

\section{The $Y$ Operator}

For the benefit of those who are not familiar with the $Y$ operator of the $\lambda$-Calculus we now present a development leading up to this operator and its purpose.

Let :

$$
(\underline{\text { Let }} f(x)=F(x, f) \text { in }
$$

define $f$ recursively -- i.e.: the right-hand side occurrence(s) of $f$ in $F(\ldots)$ denotes the same as does the left-hand side $f$.

Since, in the $\lambda$-Calculus:

$$
G=(\lambda x \cdot G)(x)
$$

provided $x$ does not occur (free) in $G$, and since let $f(x)=F(x, f)$ is the same as let $f=\lambda x . F(x, f)$ we get:

$$
(\text { Let } f=(\lambda g \cdot \lambda x \cdot F(x, g))(f) \text { in }
$$

where $g$ does not occur (free) in $F(x, f)$. If we ascribe the name $F$ to $\lambda g \cdot \lambda x . F(x, g)$, i.e. if we let: 


$$
F=\lambda g \cdot \lambda x \cdot F(x, g)
$$

then the previous can be written:

$$
(\underline{\text { Iet }} f=F(f) \text { in }
$$

Now: $F$ is a function, in fact it is a functional. And objects, say $w$, satisfying:

$$
w=H w
$$

where $H$ is any function, are said to be fixed-points of $H$. The $f$ we are looking for, i.e. defining by the let clause, thus is a fixed-point of $F$. It turns out that there may be many fixed-points of any given functional -- so, following good practice, we select one which is in some way unique; in particular, we choose to let such $f^{\prime}$ s denote the socalled minimal fixed-point. This means: that solution, $f$, (to the equation) whose graph is included (ㄷ) in any other solution. It then turns out that, under suitable and always reasonable constraints (monotonicity, etc.), there exists a functional, let us name it $\gamma$, which when applied to objects, like $F$, produce their minimal fixed-point:

This is the $Y$ we are referring to. It is the so-called minimal fixedpoint finding operatator. Observe the sequence, repeated from above:

$$
\begin{aligned}
\text { let } f(x) & =F(x, f) \\
\text { let } f & =\lambda x \cdot F(x, f) \\
\text { let } f & =(\lambda g \cdot \lambda x \cdot F(x, g))(f) \\
\text { let } f & =F(f) \\
\text { let } f & =Y F \\
\text { let } f & =Y \lambda g \cdot \lambda x \cdot F(x, g) \\
\text { let } f & =Y \lambda f \cdot \lambda x \cdot F(x, f)
\end{aligned}
$$

That is: writing $\lambda g \cdot \lambda x . F(x, g)$ or writing $\lambda f^{2} \cdot \lambda x . F(x, f)$ produces identical meanings, since the $\lambda$ 's shield the right-hand side $f$ from the lefthand side $f$-- they are now, formally speaking, not the same, although they denote the same object: 


\subsection{FunCTion Operations}

There is only defined one operation on FunCTion objects:

$$
\text { (..) apply. }
$$

\section{Example $\underline{4} \underline{2}:$}

In the introductory example we illustrated the 'concoction' of procedure denotations. In the following example we show their application. Let:

1

$$
\text { Block :: Var-set (Id } \vec{m} \text { Pro) Stmt* }
$$

be an abstract syntax (::-) xule. Block abstracts the domain of the blocks of some goto-free source language. They consist of three parts: Variable declarations, uniquely named (Id) Procedures, and a statement list. Their semantics is:

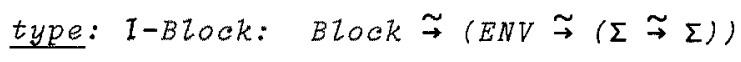

where:

2

3

4

$$
\begin{aligned}
& \Sigma=L O C \vec{m} V A L \\
& E N V=I d \vec{m} D E N \\
& D E N=L O C \quad \mid\left(A R G^{*} \sim(\Sigma \widetilde{\rightarrow} \Sigma)\right)
\end{aligned}
$$

and :

5.0

.1

.2

.3

.4

.5

.6
1-BLock $(m k-B$ Lock (vans, procm, stZ) $)(\rho)(0)=$

(Let $\left(\sigma^{\prime}\right.$, map $)=$ alzocate (vars) $(\sigma)$

Let $\rho^{\prime}=\rho+\operatorname{map}$

$+\left[i d \rightarrow V-P r c(p r o c m(i d))\left(\rho^{\prime}\right)\left(\sigma^{\prime}\right)\right.$

1 id $\in$ domprocm ]

Let $\sigma^{\prime \prime}=1-S t m t-2 i s t(s t z)\left(\rho^{\prime}\right)\left(\sigma^{\prime}\right)$

o" $>$ rng map) $\underline{i n}$

$\frac{i n}{i n}$

Lines .1-.4 constitute the block activation prologue, line .6 the matching epilogue. The function allocate is given variable names and states and produces a new state in which is allocated all the cells corresponding to the variables, their cell locations is recorded in map which 
binds variable names to these locations:

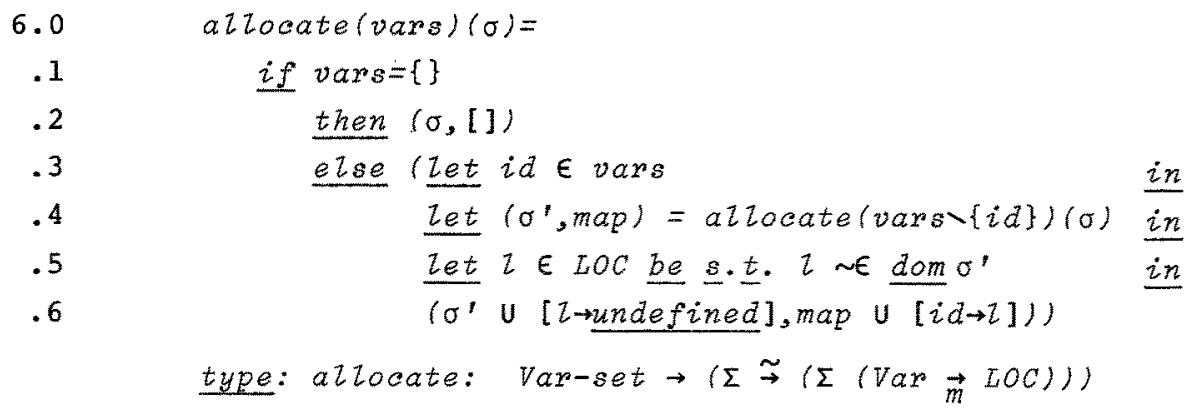

and :

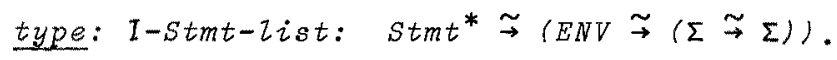

Now a statement could be a call of a procedure:

$\begin{array}{ll}7 & \text { Stmt }=\operatorname{CaZZ} \mid \ldots \\ 8 & \text { CazZ } \quad: \quad \text { Id Expr }{ }^{*}\end{array}$

and with:

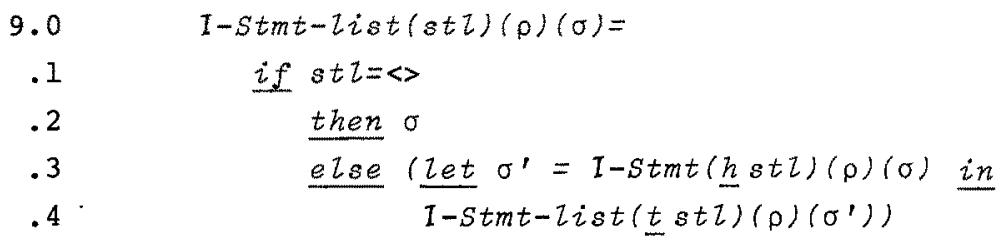

we end up having to explain:

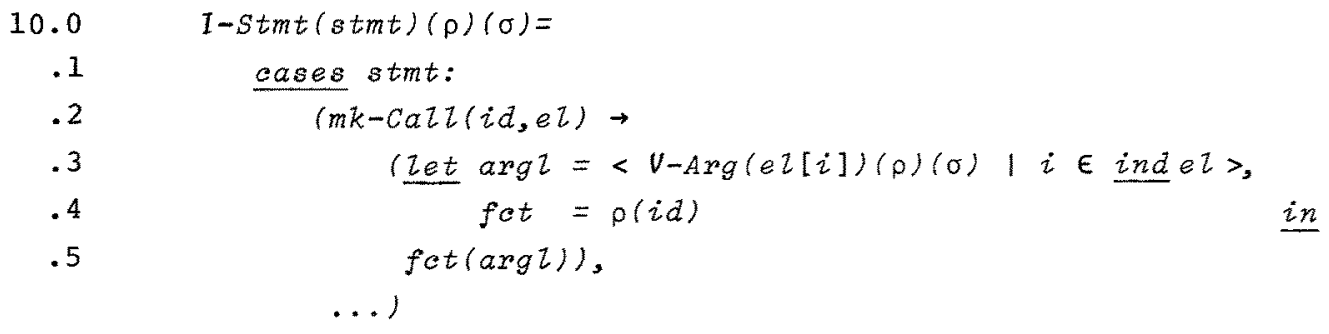

where it is assumed that:

type: V-Arg: Expr $\stackrel{\sim}{\rightarrow}(E N V \stackrel{\sim}{\rightarrow}(\Sigma \stackrel{\sim}{\rightarrow} A R G))$ 
in other words, that evaluation of the argument 2 ist does not create side-effects by changing the state $(\sigma)$.

Observe now, how the procedure denotation, which is a Function, $\left(A R G^{*} \sim(\Sigma \sim \Sigma)\right)$, "packed" in the environment $\rho$ ' set up at prologue "time", is retrieved (10.4) and applied (10.5) at "calling" time.

We have completed our task of showing the construction of Function objects, the second of the introductory examples of this section; and the application of such objects to their arguments.

Observe that the apply operator of line .4 above, retrieving fet from the MAP object $\rho$, is a MAP operation; whereas the apply operator of line .5 above, applying the Funcrion object, fot, to its argument 2 ist, is the FunCTion operation of apply. 


\section{ABSTRACT SYNTAX}

Example $4 \underline{3}:$

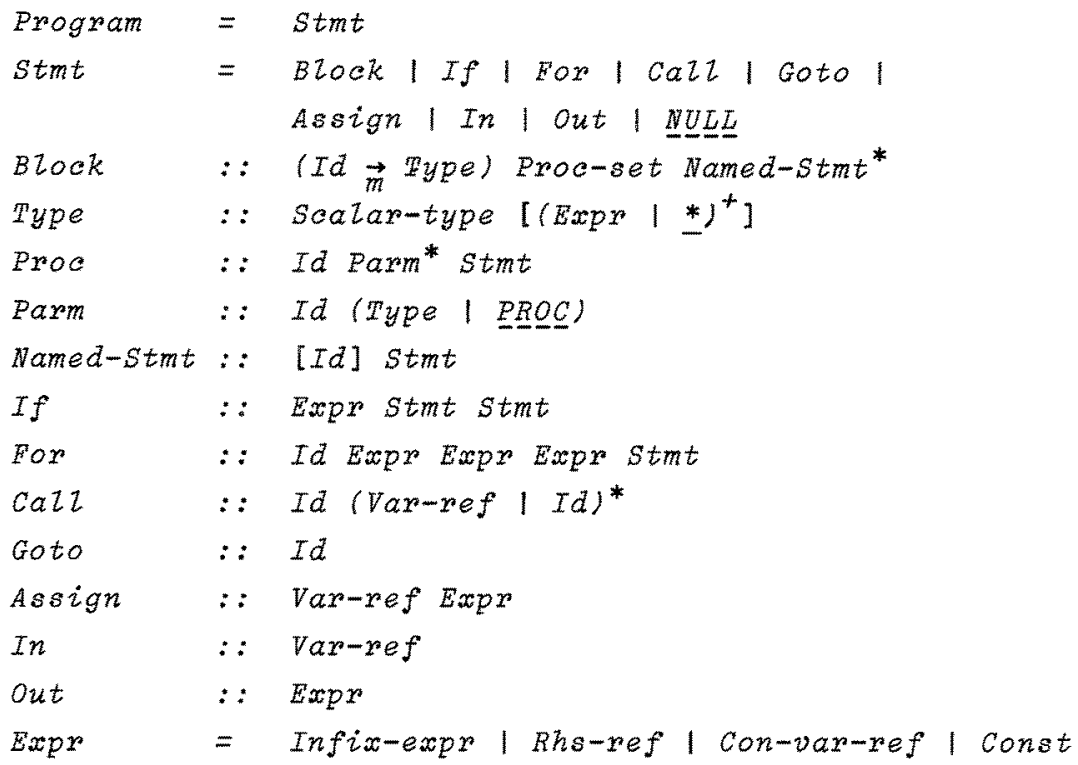

The above is an example of an incomplete(d) abstract syntax. It is intended to define fragments of the syntactic domains of some source language. Before commenting on its use of $=$ and $:$ rules; and of domain operators: $1, \vec{m}^{*}$, , set and $[\ldots]$, let us, for the sake of instruction, annotate the above syntax in the way we believe a formal model should render itself more open to inspection by casual readers:

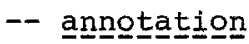

A program is a statement.

A statement is either a block, or an if, or a for, or a call, or a goto, or an assign, or an in, or an out, or a null.

A block has a variable declaration part which to variable identifiers uniquely associates the type of the values that can be stored in the variable, a set of procedures, and a list of named statements. Thus a block has three parts. And either, some, or all, of these may be void. 
A variable type is either a scalar or an array type. In this model the scalar type is modeled whenever the optional $\left[\left(\left.\operatorname{Exp}\right|^{*}\right)^{+}\right]$object is 'absent', i.e. niz. An array type has all array elements being of the same scalar type. The dimension of the axray is given by the length of the present (Expr |* $)^{+}$list.

A procedure has an identifying name, a parameter specification list, and a body which is a statement.

A parameter specification has an identifying formal parameter name and an argument type description. The type of an argument is either that of a scalar-, an array- or, a procedure.

A named statement has two parts: an optional label, which is an identifier, and a statement.

\section{Etcetera.}

\section{Comments}

From the above annotations it transpires that the domain expression "I" operator stands for "either", the paired operators "[...]" for optionality, and "(...)" delimits the scope of the infix $\vec{m}$ in rule 3 , the suf$\mathrm{fix}^{+}$in rule 4 , the infix 1 in rule 6 , and the suffix ${ }^{*}$ in rule 10.

Next we observe that the above syntax defines e.g. the domain Stmt recursively. For example: a statement is $(=)$ an if-then-else statement (2); and an if-then-else statement contains both a (then) consequence and an (else) alternative (8), both of which are statements.

Finally we conclude that the domains denoted by the rule left-hand sides must constitute some kind of solution to the mutually recursive set of equations, whether the definition symbol be "=" or "::"

\subsection{Domains of Abstract Objects}

In the following we shall rely on your returning yourself to the above example, as well as to some of the abstract syntax examples previously shown . 
The aim of section 7 is to teach you how to decompose the more general task of defining recursive classes of abstract objects. Sections 2-3-4-5-6 already taught you the more isolated construction of set, tuple, map, tree and function domains.

\section{Motivation:}

The reason why we in general, wish to define arbitrarily compounded classes is the following.

The meta-language is used, primarily, to describe complex software architectures: programming languages, data bases, operating system command \& control language interfaces; and their stepwise realization: language processors, etcetera. Just like we ordinarily describe the concrete text strings, making up e.g. programs, queries and commands, in terms of e.g. BNF grammars -- so we now desire to give abstractions of these classes of objects. Likewise: just as we, when coding a higherlevel language program, a data base data definition, etcetera, define, normally as part of variable declarations, the structure of our internal, stored objects -- so we now desire to give storage layout-independent abstractions of the more intrinsic of these value classes.

\section{Syntactic \& Semantic Domains:}

In the motivation above, two kinds of abstract object classes were singled out. The ones input to the software systems being defined, viz.: program texts, data base query \& update commands, operating system job control language commands, etc. And the ones manipulated, and, as we shall take it in general, denoted by these inputs, viz.: internal data structures; catalogues, files \& records; processes, resources, ercetera. We shall generally refer to the former object classes as being of syntactic nature, and the latter as being semantic. Syntactic and semantic domains will, in the meta-language be defined using the programing construct of abstract syntax.

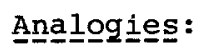

The "programming construct" of BNF grammars or some extended variant thereof is usually applied when defining sets of concrete text strings 
-- i.e. context-free "languages". And the type and mode, definition facility of PASCAL, respectively ALGOL 68, is the corresponding programing construct for the internal data structures. In the meta-language the same tool will be applied in the construction of potentially, and usually, infinite classes, or as we shall prefer to call them, domains of abstract objects.

\section{Motivivation:}

The reason why we apply the same tool and why this tool is neither that of e.g. BNF, the PASCAL type definition nor the ALGOL 68 mode definition facility, is that we, on one hand, neither want to deal with text strings (or corresponding parse trees), nor, on the other hand, are concerned about storage space and access-efficient layout of objects. Instead we desire to provide what we believe to be appropriate fitting representational abstractions.

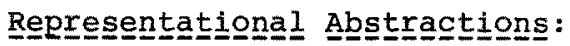

By representational abstraction is meant the target system (e.g. input/ output terminal-, or machine-storage device) independent specification of software function concepts, especially objects, emphasizing the choice of such abstractions whose, usually composite, (logical) type most directly expresses intrinsic, i.e. to our understanding relevant, properties of the notions being specified.

\section{Domain Definitions $\underline{\text { Compositions }}$}

In order:

(1) to decompose the task of constructing domains, i.e. of composing them from constituent domains, and in order

(2) to permit the constructions of reflexive domains, i.e. composite domains whose objects contain properly embedded objects of the same kind (-- or domain)

the meta-language provides the conventional notion of definitions. 


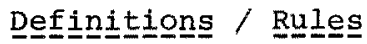

A domain definition consists, as does any definition, of two parts. A left- and a right hand side. Other words are 1hs, respectively rhs; and definiendum ("that which is being defined"), respectively definiens ("that which defines it"). We shall use the words abstract syntax rule synonymously with the concept of a domain definition.

Abstract syntax rules all have their $1 \mathrm{hs}$ 's being simple names, i.e. identifiers. The rhs's are usually compound expressions henceforth referred to as logical type, or domain expressions.

The first point, above, about decomposing the task of defining domains is achieved by the ability to use, in the rhs's of some rules, the lhs identifier of some other rule. The second point, then, is achieved by permitting the use, in the rhs of any rule, of the lhs identifier of that (or those) rule(s).

\section{Composittions / Domain Expressions}

In order:

(3) to model notions of software systems representationally abstract, and in order

(4) to provide a reasonably fitting variety of choices for abstracting objects

the meta-language centers its abstract compound objects around the

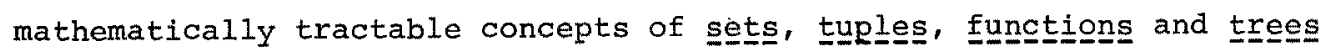
together with associated, primitive operations.

\section{Domain Operators :}

As a consequence the meta-language provides a number of operations which apply to arbitrary (constituent, elementary \&/or composite) domains to denote set-, tuple-, map-, function- and abstract tree domains.

These operators have already been introduced, and are:

$$
-\operatorname{set} ; *,+\vec{m}, \stackrel{\rightarrow}{\rightarrow} \rightarrow ;(\ldots)
$$

the latter $((\ldots))$ denoting (implicit or anonymous) tree construction. 
In the compounding, usually signalled by the implicit, or anonymous, tree constructor $(\ldots$.$) , of more than one domain:$

$$
\left(\begin{array}{llll}
D_{1} & D_{2} & \cdots & D_{n}
\end{array}\right) \quad n \geq 2
$$

there is an invisible "cartesian product-like" operator, $x$. That is, you might read the above as:

$$
\left(D_{1} \times D_{2} \times \ldots \times D_{n}\right)
$$

The point about the use in this meta-language of the cartesian operation is that it is only used when domains of trees of two or more subcomponents are denoted. Not, e.g., when tuple domains are to be constructed. These can only be constructed, in this meta-language, using the * or * operations. Thus, where context permits, we may drop implicit tree constructors, as in:

$$
A \rightarrow B C
$$

which is taken to be identical to:

$$
A \rightarrow(B \quad C)
$$

That is: the precedence of $x$ is higher than any other infix operator, but lower than any suffix operator. We usually omit writing the $x$ operator.

The following domain operators assemble not necessarily disjoint domains into 'unions' of these:

$$
1 \underline{U}
$$

-- the latter applicable only in connection with map domains, as explained in section 4.1. The 1 operatox will be further defined below.

Finally the operators:

$$
\begin{aligned}
& \text { [...] - optionality } \\
& \text { (...) - - grouping/delimiting }
\end{aligned}
$$

enables shortness of descriptions. The former:
$[A]$
A $\mid$ nit. 


\section{The Scope Delimiting (_... Operator}

The latter can easily be confused with the tree construction operator. Therefore: when (...) surrounds two or more domain expressions with no infix domain operator, other than the cartesian operator, separating them, e.g. as in:

$$
\left(A^{*} B-\operatorname{set} C\right) \text {, }
$$

then $(. .$.$) denotes tree domain construction; otherwise (...) as in :$

$$
\left(A^{*} \mid(B-\operatorname{set} \rightarrow \vec{m})\right)^{*} \text {, }
$$

serves to indicate, i.e. delimit, the scope of infix or suffix domain operators. The corresponding scope rules are the conventional ones.

\section{Infix Operator Commutativivity and Associativivity}

With infix operators the question of their commutativity and associativity arises:

$$
\begin{aligned}
& 1, \frac{U}{\sim}, \quad \text { are commutative } \\
& \vec{m}, \rightarrow \quad \text { associates to the right. }
\end{aligned}
$$

The cartesian product operator, $x$, does not associate!

\section{Examples $4 \underline{4}-\underline{4}-\underline{5}-\underline{4} 6$ :}

(1) The domains denoted by:

$$
A \mid B \text { and } B \mid A
$$

are identically the same, and so are:

$$
\begin{aligned}
& (A \vec{m} B) \underline{U}\left(C \vec{m}^{D} D\right), \text { and : } \\
& (C \underset{m}{\rightarrow} D) \underline{U}(A \underset{m}{B} B) .
\end{aligned}
$$

(2) The domains:

$$
\begin{aligned}
& A \rightarrow(B \rightarrow(C \rightarrow D)), \text { and : } \\
& A \rightarrow B \rightarrow C \rightarrow D
\end{aligned}
$$

are identically the same. Writing the former is a clarification of the 
latter, should you have forgotien the rules of associativity. Thus the domain:

$$
((A \rightarrow B) \rightarrow C) \rightarrow D
$$

is distinct from the domain denoted by the former expressions.

(3) The tree domains:

$$
(A \times B \times C),((A \times B) \times C) \text {, and }(A \times(B \times C))
$$

are all distinct (in fact, disjoint). Only in this, the 3rd example, was $(.$.$) used for tree domain construction.$

\section{Semantics of the 1 Operator}

of the new domain expression operators $([\ldots],(\ldots), 1)$ introduced in section 7 only the meaning of $\mid$ remains to be explained.

Let $A$ and $B$ be domain expressions, denoting $A$, respectively $B$. Then:

$$
A \mid B
$$

denotes :

$$
A \cup B
$$

where $U$ is the (potentially infinite set) union operator; i.e.:

$$
A \mid B \quad \triangle \quad\{0 \mid O \in A \vee O \in B\}
$$

\section{Comment:}

Thus 1 does not denote the discriminated or disjoint union operation.

In scott 76 the disjoint union operator is represented by + , and:

$$
\begin{aligned}
A+B \Delta \Delta & \{(\underline{A}, a) \mid a \in A\} U \\
& \{(\underline{B}, b) \mid \in B\}
\end{aligned}
$$


The "tagging" of the $A+B$ domain elements, with a "label" indicating the originating domain, is useful whenever (1) domains $A$ and $B$ overlap, and (2) the need arises for testing, say in some function, whether an $A+B$ object comes from $A$ or from $B$.

Since the I operator does not separate the operand domains it is the task of the definer to see to it that I operand domains are disjoint whenever some functions applicable to the union domain need ascertain originating domain.

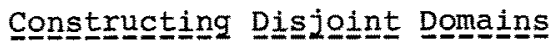

Let us assume that a union domain, roughly of the type:

$$
D_{1}\left|D_{2}\right| \ldots \mid D_{n}
$$

is required. Let us further assume that for some, or all, $i \neq j$ :

$$
D_{i} \text { is not disjoint from } D_{j}
$$

Since $D_{i}$ and $D_{j}$ overlap there is no direct way of separating (some) $D_{i}$ objects from $D_{j}$.

Introducing the abstract syntax tree domain constructing rules:

$$
\begin{aligned}
& A_{1}:: D_{1} \\
& A_{2}:: D_{2} \\
& \ddot{A}_{n}:: D_{n}
\end{aligned}
$$

and :

$$
A_{1}\left|A_{2}\right| \ldots \mid A_{n}
$$

(in lieu of $\left.D_{1}\left|D_{2}\right| \ldots \mid D_{n}\right)$, effectively corresponds to a distinct marking $\left(m k-A_{1}, m k-A_{2}, \ldots, m k-A_{n}\right)$ of respective $D_{1}, D_{2}, \ldots, D_{n}$ objects in the union domain $A_{1}\left|A_{2}\right| \ldots \mid A_{n}$. Distinctness is solely achieved by distinctress of left-hand side $\left(A_{i}, A_{j}\right)$ names.

Thus we introduce : : abstract syntax rules whenever disjointness is desired. 
7.2 Abstract Syntaxes \& Rules

\section{Abstraat Syntaaeㅗ}

$\begin{array}{ll}\text { Abstract-syntax } \quad::=\quad \text { Rule } & \\ & \text { Rule } \\ & \cdots \\ & \text { RuIe }_{r}\end{array}$

is an informal "extended" BNF, or BNF-like, syntactical description defining an abstract syntax as consisting of a number of rules. Each rule:

$\begin{array}{lll}\text { Rule } & := & \text { Identifier }=\text { Domain-Expression } \\ ::= & \text { Identifier : Domain-Expression }\end{array}$

basically consists of two parts: a left-hand side definiendum, which is an identifier; and a right-hand side definiens, which is a domain expression. The two rule parts are separated either by an equality ( $)$ operator or by the so-called tree domain constructor $(: ;)$ operator. Both the $=$ and the $:$ operators are definition symbols (like: $\Delta$ or $\mathrm{d} \overline{\bar{e}} f$ ). The latter, in addition, 'constructs' trees. Its use in:

$$
A \quad: \quad B_{1} B_{2} \cdots B_{n}
$$

could be taken as an abbreviation for the (intended, reflexive) use of $\therefore$ in:

$$
A=\quad \therefore\left(B_{1} B_{2} \ldots B_{n}\right)
$$

\section{Context Constrraint}

In a complete meta-language program there must be no two rules of any one or pair of abstract syntaxes with identical definienda, i.e. Ihs identifiers.

\section{tis-Functíng}

Any abstract syntax rule implicitly introduces, i.e. defines, a predicate function is-Identifier: 
type: is-Identifier: $O B J \rightarrow B O O L$

which applies to any object and yields true if it is an object in the domain denoted by Identifier, otherwise fatse.

\section{Noㅡㅌㅗ:}

We could have chosen to write:

$$
o b j \in \text { Identifier }
$$

instead of:

$$
\text { is-Identifier }(o b j)
$$

but, except for a few cases, we reserve the former form for set membership tests where the set is not one defined as a Domain as defined by some abstract syntax. The exceptions are those illustrated by the following descriptor and quantified expressions (see sections 1.5 \& 1.4.1):

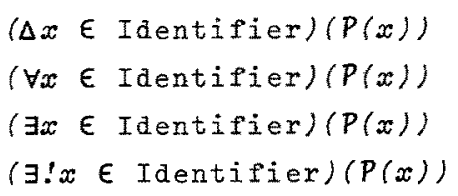

\section{Example 47:}

Given the introductory abstract syntax example, and given: is-Stmi(s), is-ID(id), is-Scalar Type(st), is-Named-Stmt(ns1), is-Named-Stmt(ns2), $i s-S t m t(s 1), i s-S t m t(s 2)$, and $i s-E x p r(e)$ we are able to construct:

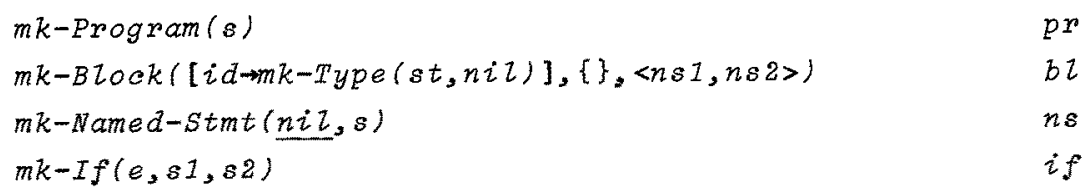




\begin{tabular}{|c|c|}
\hline$i s-$ Program $(p r)$ & $\sim i s$-Program $(b z)$ \\
\hline$\sim i s-s t m t(p r)$ & $i s-S t m t(b l)$ \\
\hline$\sim i s-B$ Lock $(p r)$ & $i s-B \operatorname{lock}(b l)$ \\
\hline$\sim i s-$ Named-Stmt $(p r)$ & $\sim i s-N a m e d-S t m t(b l)$ \\
\hline$\sim i s-I f(p r)$ & $\sim i s-I f(b z)$ \\
\hline$\sim i s-\operatorname{Prog} r a m(n s)$ & $\sim i s-P r o g r a m(i f)$ \\
\hline$\sim i s-S t m t(n s)$ & $i s-S t m t(i f)$ \\
\hline$\sim i s-B$ lock $(n s)$ & $\sim i s-B$ lock $(i f)$ \\
\hline is-Named-stmt (ns) & $\sim$ is-Named-Stmt (if) \\
\hline$\sim i s-I f(n s)$ & $i s-I f(i f)$ \\
\hline
\end{tabular}

etc. .

\section{Furthher Context Constrxainnt}

$A=-r u l e$, when expressible in the form:

$$
\text { Identifier }=D_{1} D_{2} \cdots D_{n} \quad(n \geq 2)
$$

is identical to the rule:

$$
\text { Identifier } \quad:: D_{1} D_{2} \ldots D_{n} \quad(n \geq 2)
$$

\section{Semantics of Abstract Syntaxes}

The prescription for computing most of the domains designated by compound domain expressions has already been given. Allowing now for domain definitions, i.e. rules, in fact for several such, and, in addition, for potentially mutually recursive rules, we require a more comprehensive prescription for computing the domains given an arbitrary abstract syntax. We shall only do that informally here, relying in general on the formal foundations laid by scott. [Scott 1976].

An abstract syntax can, speaking rather formally, be viewed syntactically as an equation set. Its meaning is a family of named sets of mathematical objects being the minimal, fix-point solution to the equation set. That is: to each $\mathrm{hhs}$ there corresponds a potentially infinite class of finite objects, the domain. These domains are the smallest such classes which, when substituted in lieu of their identifying names 
in the equation Ihs and rhs's will, satisfy the equations. The bit about the fix-point comes into the picture since our equations may be mutually recursive.

Example 4 으:

The equation:

$$
D=D \rightarrow \vec{m}
$$

has the following solution:

$$
D:\{[],[[] \rightarrow[]],[[] \rightarrow[[] \rightarrow[]]],[[[] \rightarrow[]] \rightarrow[1], \ldots\} ;
$$

with :

$$
D=(D \vec{m} D) \mid A
$$

"adding" :

$$
\begin{aligned}
& {\left[\left[a_{1} \rightarrow[1], \ldots\right.\right.} \\
& {\left[[] \rightarrow a_{1}\right], \ldots} \\
& {\left[a_{1} \rightarrow a_{1}\right], \ldots} \\
& {\left[a_{1} \rightarrow a_{1}, a_{2} \rightarrow a_{2}, \ldots, a_{n} \rightarrow a_{n}\right], \ldots} \\
& \ldots\}
\end{aligned}
$$

to the above solution. 


\subsection{Abstract Syntax oriented Combinators}

The meta-language (structured-) expression and statement combinators specifically complementing the (abstract syntax) domain expression alternative operator, 1 , are:

The McCarthy Conditional Cla

$$
\begin{aligned}
& \left(p e_{1} \rightarrow c_{1},\right. \\
& p e_{2} \rightarrow c_{2}, \\
& \cdots \\
& \left.p e_{n} \rightarrow c_{n}\right)
\end{aligned}
$$

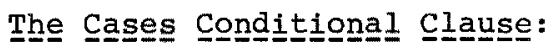

$$
\begin{aligned}
\frac{\operatorname{cases}}{\left(e_{1}\right.} & \rightarrow c_{1}, \\
e_{1} & \rightarrow c_{2}, \\
\cdots & \\
e_{n} & \left.\rightarrow c_{n}\right)
\end{aligned}
$$

Here $p e_{i}$ stand for predicate expressions, $a_{j}$ for either expressions or statement, and $e_{k}$ for expressions. A clause is either a statement or an expression. If either of the above conditional clauses is intended to be) of the expression type, then all $c_{j}$ are (to be) expressions. Similarly they are all to be statements if the above clauses are to be conditional statements. We refer to the $e_{k}$ expressions as follows: $e_{0}$ as the root expression and $e_{k}$, for $1 \leq k \leq n$, as the branch expressions.

\section{Example $\underline{4}$ ㅇ:}

Given the abstract syntax for expressions of some source language: 


\begin{tabular}{|c|c|c|c|}
\hline 1 & $E x p x^{2}$ & $=$ & Infix | Rhs-ref | Con-var-ref | Const \\
\hline 2 & Infix & $\therefore:$ & Expr op Expr \\
\hline 3 & Rhs-ref & $::$ & Var-ref \\
\hline 4 & Con-var-ref & $\therefore:$ & $I d$ \\
\hline 5 & Const & $=$ & $I N T G \mid B O O L$ \\
\hline 6 & Var-pef & $::$ & $I d\left[E x p p^{+}\right]$ \\
\hline 7 & $O p$ & $=$ & Int-Op | BOOl-Op | Ret-Op \\
\hline
\end{tabular}

We can define a function, ex-tp, which given a dictionary:

$\begin{array}{llll}8 & \text { DICT } & =I d \vec{m} \text { (Type }|\underline{P R O C}| \ldots) \\ 9 & \text { Type } & : \text { Scalar-type }[(\text { Expr } \mid *)+] \\ 10 & \text { Scalar-type } & =\text { INI } \mid \text { BOOL }\end{array}$

computes the Scalar-type of an expression, $e$ :

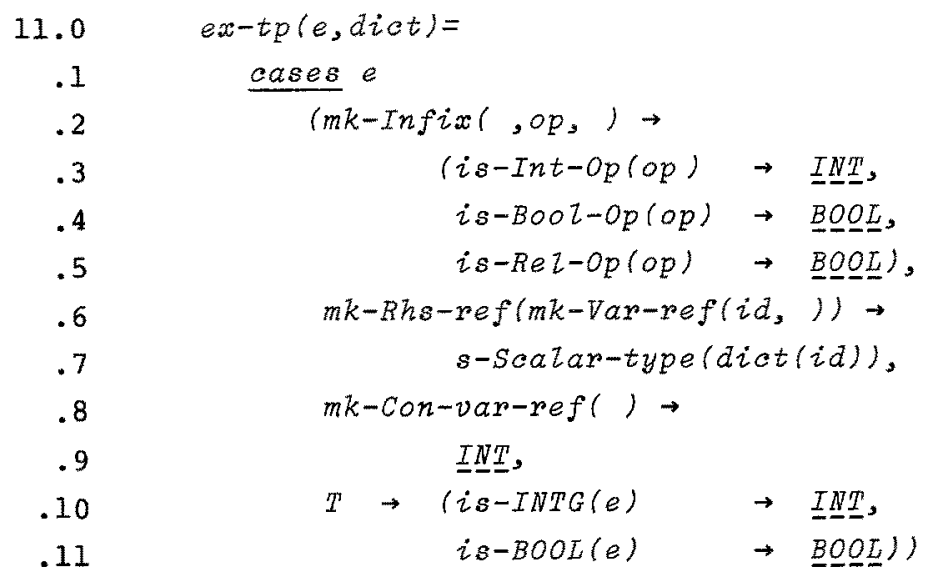

type: Expr DICT $\rightarrow$ Scalar-type

Observe how, in this case, we nested the Cases and the McCarthy constructs. Relate the structures of the abstract syntax and the ex-tp function, and observe how they "match". Observe next the use of mkconstructs in the cases branch expressions. Observe finally that we only name those arguments of the $m k$ - functions which we explicitly require.

\section{-- annotatitions:}

An expression is either an infix expression, a right-hand-side reference, a controlled variable reference or a constant. 
An infix expression has three parts: two operand expressions and an operator.

A right-hand-side variable reference is a variable reference.

A controlled variable reference is an identifier.

A constant is either an integer or a boolean.

A variable reference has an identifier and, if this identifier denotes an array location, then an index list, which is a non-zero, finite length expression list, else nil.

An operator is either an integer (arithmetic) operator, a boolean operator, or a relational operator.

\section{-- comments:}

An expression either denotes an integer or a boolean. Hence the type of an expression is (said) either (to be) INT or (to be) BOOOL. The type of an expression -- which is otherwise well-formed -- can be statically ascertained. Given that the type with which a variable or formal parameter is declared:

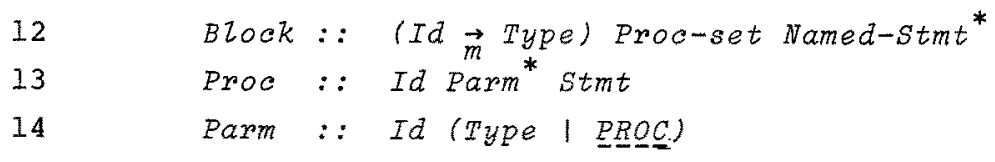

is recorded in some (compile-time) dictionary, also called static environment, the function ex-tp computes (at compile-time) the type of any expression.

\section{-- annnotatitions, continued:}

ex-tp is given an expression, e, and a dictionary, dict.

If $e(.1)$ is an infix expression (.2), i.e. if e can be expressed as some $m k-I n f i x(e 1, o p, e 2)$, where $e 1, o p$ and $e 2$ become the names of those Expr, $O p$, respectively Expr objects of which $e$ is made up, then the type of $e$ is ascertainable from the kind of operator that $o p$ is. If $o p$ is .3) an integer operator, then the type of $e$ is $I N T$; if op instead is either (.4) a boolean or (.5) a relational (or comparison) operator, then the type of $e$ is BOQL.

If $e(.1)$ is a right-hand side reference (.6), i.e. if e can be expressed as some $m k-R h s-r e f(v r)$, where $v_{r}$ is a variable reference (meta-) expressible as some $m k-V a r-r e f(i d, e l)$, where $i d$ and $e l$ become the names of the Id, respectively [Expr $\left.{ }^{+}\right]$objects of which $e$ can be made up, then the type of e can be looked up in the dictionary as that 
of the scalar-type component of the Type object with which id is associated.

\section{PART III COMBINATORS}

In part II, i.e. in sections 2-7 inclusive, we dealt with all aspects of constructing domains of objects, constructing objects and performing primitive, i.e. language-defined operations on objects.

Certain desired transformations on objects are, however, of a complexity which cannot easily be described by some such operator/operand expression, regardless of its composition. To that end, and as is quite customary in programming, the meta-language provides a number of constructs which facilitate the gradual composition of transformations and processes on objects. We call these constructs for combinators. They are:

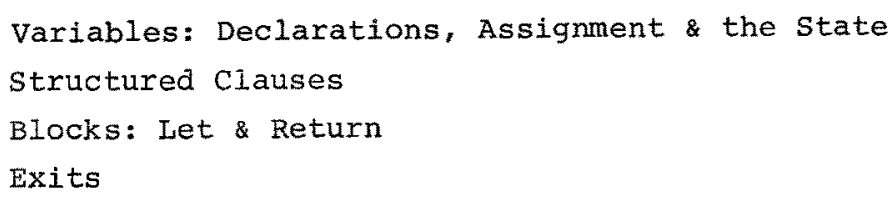

So far we have officially dealt only with the applicative aspects of the meta-language. Introducing variables implies introducing imperative constructs, i.e. statements. The applicative part of the language, however, includes the let constructs of, and hence also, blocks, as well as most of the structured clauses. We say, in general, that the applicative constructs of the language permit the expression of composite transformations on objects. The imperative constructs correspondingly enable the decomposed, stepwise statement of processes on objects. 


\section{VARIABLES}

Example $\underline{50}$ :

The following four function definitions all define $f_{i}$ to denote the fac torial function:

$1 f_{0}(n)=$ if $n=0$ then 1 else $n * f_{0}(n-1)$

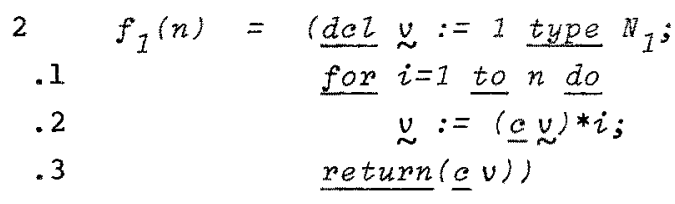

$3 \quad f_{2}(n)=\left(\underline{d c l} \underset{\sim}{v}:=1\right.$ type $N_{1}$;

$.1 \quad$ for $a I z i \in\{1: n\}$ do

$.2 \quad \stackrel{v}{\sim}:=(\underline{a} \underset{\sim}{\sim}) * i ;$

$.3 \quad$ return $(\underline{e})$

$4 f_{3}(n)=\left(\right.$ del $\underset{\sim}{v}:=1$ type $N_{1}$,

$.1 \quad \underset{\sim}{i}:=n$ type $N_{0}$;

.2 while $(c i \neq 0)$ do

$.3 \quad(\underline{\sim}:=(\underline{e} \underset{\sim}{u}) *(\underline{e} \underset{\sim}{i})$;

$.4 \quad i:=(e \underset{\sim}{i})-1)$;

$.5 \quad$ return $(c v))$

The first defines $f_{i}$ applicatively; the remaining three, imperatively.

We say that the parenthesized constructs: (del ... return (...) ) are blocks. $\underset{\sim}{u}$ and, only in the last definition, $\underset{\sim}{i}$ are (assignable) variables. They form part or all of the state current in these blocks. If no other (externally declared) variables 'exist', then they form ali of the state. The logical type of the four $f^{\prime} s$ are:

$$
\text { type: } \quad f_{0}: \quad N_{0} \rightarrow N_{1}
$$




$$
\text { type: } f_{1}, f_{2}, f_{3}: N_{0} \rightarrow\left(\Sigma \rightarrow\left(\Sigma N_{1}\right)\right)
$$

where $\Sigma$ denotes the state space. The contributions of $\underset{\sim}{v}$ and $\underset{\sim}{i}$ inside the blocks, to this space is:

$$
\Sigma=\cdots \underline{u}\left(\underset{\sim}{\underline{m}} N_{1}\right) \underline{u}\left(\underset{\sim}{i} \vec{m}_{0}\right)
$$

where the ... ellipsis refers to possibly externally declared variables. Since none of the $f_{1}, f_{2}$ nor $f_{3}$ assign to any such global variables it is easy to see that they do not alter any global state, and hence that the potential state transformation indicated by:

$$
\Sigma \rightarrow \Sigma
$$

is in fact the identity change. Thus the four functions can indeed be shown 'equivalent' even when considering a global state.

\subsection{Declarations \& The State}

Although the examples above featured block local declarations we shall normally not find. a need for other than global variables in abstract models of higher-level software.

The meaning of a declaration:

$$
\text { del } \underline{v a r}:=\ldots \text { type } D
$$

-- in which we may omit the type $D$ clause -- is that of joining to our state, $\sigma$, a contribution:

$$
\sigma \quad U[\underbrace{\operatorname{Var}}_{\sim a r} \rightarrow o b j]
$$

It is understood that $\mathrm{Van}_{\mathrm{n}}$ is not already declared, i.e. that Van redomo. In general, the state space, $\Sigma$, given a collection of (global) declarations:

$$
\begin{aligned}
\operatorname{del} \underset{v_{1}}{v_{1}} & :=\ldots \text { type } D_{1}, \\
{\underset{\sim}{v}}_{2} & :=\ldots \text { type } D_{2}, \\
\ddot{v}_{n} & :=\ldots \text { type } D_{n} ;
\end{aligned}
$$


can be defined as:

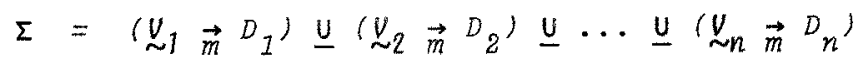

The domain expressions:

$$
\left(\underset{\sim}{V_{i}} \vec{m} D_{i}\right)
$$

are degenerate in that the denoted map domains are singular, i.e. consist of just one element, the (quotation-like) object $\underset{\sim}{V}{ }_{i}$ which can be regarded as denoting itself.

\section{Example 51:}

Our source language example features the ability to input data from an (one) external device, to output data to an (one) external device -distinct from the input device, and to declare variables in any (nested) block. The relevant syntactic domains, continuing example 31 , are:

$$
\begin{aligned}
& \text { Block } \quad: \quad \text { (Id } \vec{m} \text { Type) ... Stmt } \\
& \text { Stmt } \quad=\quad \text { In | Out | Assign | .. } \\
& \text { In } \quad \therefore \quad \text { Var-ref } \\
& \text { Out } \quad \therefore \quad \text { Expr } \\
& \text { Assign }: \therefore \quad \text { Var-ref Expr }
\end{aligned}
$$

The semantic functions ascribing meaning to these source constructs will be based on the following three (global) variables:

$$
\begin{aligned}
& \text { deI Input }:=\langle\ldots\rangle \text { type }(I N T G \mid B O O L)^{*} \text {, } \\
& \text { Output := } \Leftrightarrow \text { type (INTG|BOOL)*, } \\
& \text { STG }:=11 \text { type } S T G \text {; }
\end{aligned}
$$

where STG was defined in the last example of section 4.4 .

The state space, $\Sigma$, of the elaboration functions, is:

$$
\begin{aligned}
& \Sigma=\left(\text { Input } \vec{m} \quad(\text { INTG|BOOL })^{*}\right) \underline{U} \\
& \text { (Output } \vec{m} \text { (TNTG|BOOL)*) } \underline{\mathrm{u}} \\
& \text { (STG } \vec{m} S T G \text { ) }
\end{aligned}
$$




\subsection{Variable References}

\section{Preludude}

Recall the introductory examples of section 8. Variable identifiers (V) occurred in two contexts:

$$
\underset{\sim}{v}:=\ldots \subseteq \underset{\sim}{v} \ldots
$$

The left-hand side occurrence denotes itself, i.e. the meta-storage (state) location. The right-hand side occurrence of $\underset{\sim}{v}$ likewise denotes itself : But here we traditionally expect the value kept in the $\underset{\sim}{V}$ loca tion. The meta-language breaks with this tradition. If you need the content, then you are required to perform the $\in$ operation to $\underset{\sim}{V}$.

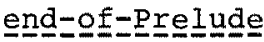

If $\underset{\sim}{\cup}$ is the name of a declared variable:

$$
\underline{\text { dcl }} \underset{\sim}{v}:=\ldots \text { type } D
$$

then $\underset{\sim}{V}$ is said to denote itself, or if need arises for more precision, to denote a ref $D$ object, i.e. a reference to an object of type $D$.

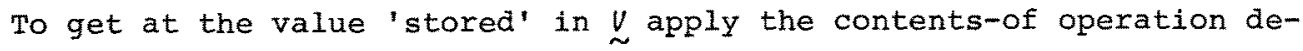
noted by $\subseteq$ :

\section{$\stackrel{e V}{\sim}$}

$\underset{\sim}{V}$ and $\subseteq \underset{\sim}{V}$ are meta-language expressions. Given the state, $\sigma:$

$$
\sigma=[\underset{\sim}{V} \rightarrow o b j, \ldots]
$$

$\underline{\sim} \underset{\sim}{V}$ is explained as :

$$
\sigma(\underline{U})
$$

If $\underset{\sim}{V}$ is of type ref $D$, then $\underline{\underline{v}} \underset{\sim}{V}$ is of type $D$. That is: $\underline{c}$ de-referen ces $\underset{\sim}{V}$. Thus $\subseteq$ denotes a function from state variables and states to objects; which we generalize to a function from state-variable and states to states 'paired' with objects: 


$$
\begin{aligned}
& \underline{e} \sim \lambda v \cdot \lambda \sigma \cdot(\sigma, \sigma(v)) \\
& e \quad \epsilon \quad(V \rightarrow(\Sigma \approx(\Sigma O B J)))
\end{aligned}
$$

\section{$\underline{8.3}$ Assignment}

Assignment is a meta-language statement:

$$
\underset{\sim}{V}:=\operatorname{expr}
$$

If $\underset{\sim}{v}$ is of type ref $D$ then expr must be of a type $D^{\prime}$, included in $D$, i.e. $D^{\prime} \subseteq D$, e.g. $D \equiv D^{\prime}$

The meaning of assignment is as you think it. Formally, however:

$$
\begin{aligned}
& :=\sim \lambda v \cdot \lambda o b j \cdot \lambda \sigma \cdot(\sigma+[v \rightarrow o b j]) \\
\text { i.e. } \quad & :=E \quad(V \rightarrow(O B J \rightarrow(\Sigma \cong \Sigma)))
\end{aligned}
$$

\section{Example 52:}

We now conclude the examples of sections $4.4,6.3$ and 8.1 . Recall in particular the last example of section 4.4 .

The elaboration functions ascribing meaning to the source language In, out and Assign statements will themselves employ, but now, meta-language assignments. Since the basic source language means of referring to a variable is through:

$$
\text { Var-ref :: Id [Exp } \left.x^{+}\right]
$$

we define a set of auxiliary functions:

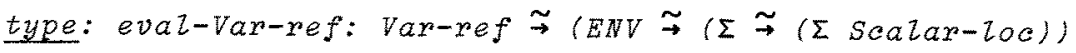

$$
\begin{aligned}
& \text { contents: } \quad \text { Scalar-2oc } \sim(\Sigma \sim(\Sigma(I N T G \mid B O O L)) \\
& \text { assign: } \quad \text { Scalar-loc }(I N T G \mid B O O L) \cong(\Sigma \cong \Sigma)
\end{aligned}
$$

eval-Var-ref takes a variable reference, an environment and a state, and produces the same state and the scalar location denoted by the variable reference. The need for accessing the state arises as a result 
of potentially evaluating array variable subscripts. Whereas eval-Var-ref applies to a syntactic object (Var-ref), and hence needs the environment; contents and assign apply only to semantic objects, and hence do not require the environment. Finally: objects assigned to storage locations must have their type match the type of the location. Recall that the storage model of the last example of section 4.4 distinguished between integer- and boolean locations:

0

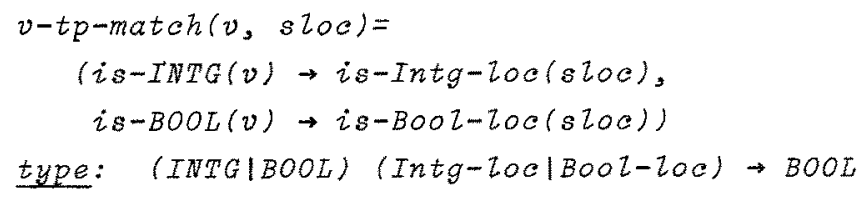

Now:

1. $\operatorname{int}-\operatorname{In}(m \hat{k}-\operatorname{In}(v p))(\rho)=$

.1 if IInput $=<$

.2 then error

.3 else (let sloc: eval-Var-ref(vr)(p),

.4 iv : ha input;

.5 if $v-t p-\operatorname{match}(i v$, s. 00$)$

.6 then (Input := tisc Input;

.7 assign(stoc,iv))

.8 else error

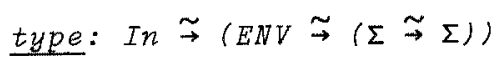

2. int-out (mk-out (e)) $(\rho)=$

.1 (let ov : eval-Expr $(e)(p)$;

.2 output $:=($ coutput $)-r\langle 0 v\rangle)$

type: out $\stackrel{\sim}{\rightarrow}(E N V \stackrel{\sim}{\rightarrow}(\Sigma \sim \Sigma))$

3. $i n t-A \operatorname{sigh}(m k-A \operatorname{ssign}(v r, e))(\rho)=$

.1 (Let sloo: eval-Var-ref(vr)( $\rho)$,

.2 val : eval-Expr(e)(o);

$.3 \operatorname{assign}(s i o c, v a t))$

type: $A s \operatorname{sign} \cong(E N V \cong(\Sigma \stackrel{\rightarrow}{\rightarrow}))$ 
The auxiliary functions:

4. $e v a l-V a r-r e f(m k-V a r-r e f(i d, s s l))(p)=$

$.1 \quad$ if $s s i=\underline{n i}$

.2 then return $(\rho(i d))$

.3. else (let arrioc $=\rho(i d)$ in

.4 Letil : <eval-Expr(ssi[i])(o) $\mid 1 \leq i \leq$ lenssi>;

.5 if it ne domarrioc

.6 then error

.7 else return(arroc(il)))

5. contents $(2)=$

. I (Let $v:$ (c STG)(i);

.2 if $v=$ undefined

.3 then error

.4 else return(v))

6. $\operatorname{assign}(2, v)=$

$.1 \quad$ STG $:=c$ STG $+[\tau \rightarrow v]$

\section{-- annotatítons}

1. Interpreting an Input statement proceeds as follows: (.1) If there is no more Input, then (.2) interpretation halts, otherwise $(.3-.8)$ we evaluate (.3) the scalar location denoted by the variable reference constituting the Input statement and (.4) retrieve the front input stream value. If $(.5)$ the type of this value matches that of the scalar location then $(.6-.7)$ we proceed to (.6) 'shorten' the Input stream by the element value just retrieved, and (.7) to assign this value to the scalar storage Zocation; otherwise interpretation halts.

2. Interpreting an output statement consists, rather nore simply, of (.1) evaluating the expression it is composed of and (.2) of appending the resulting output value to the output stream. 
Etc. for 3., int-Assign.

4. Evaluating a variable reference which consists of a possibly void array subccript $i$ ist and the variable identifier proceeds as follows: (.1) If the subscript list is absent, then (.2) the identifier is guaranteed, by well-formedness context conditions not shown, to denote a scalar variable, whose location can be obtained from the environment directly. (.3-.7) If instead the subscript Iist is present, then (.3) the identifier denotes an array variable whose location is kept in the environment. The subscript ist is guaranteed to consist of proper expressions all yielding integer values. Well-formedness context conditions for this are not shown, but see [Jones 1978a]. We therefore (.4) evaluate all the subscript expressions to obtain an index $l_{\text {ist. }}$ (.5) If this index $l_{\text {ist }}$ is not one of the array, then (.6) interpretation fails, otherwise the denoted scalar location is obtained by applying the array location, which is a map from index lists to scalar locations, to the computed index list.

\section{Comments}

In the above definitions certain 'pairs' of evaluations proceeded in "parallel": 1.3-1.4, 3.1-3.2. In [Jones 1978a] the same evaluations proceed sequentially, in the order listed. This gives rise to two distinct semantics!

\subsection{Derived References}

Section 8.2 dealt only with simple variable references. That is: references denoting the location of an 'entire' variable. In the design and use, since 1973, of the meta-language, a need, when abstracting software, has not been registered for easily denoting references to proper components of store composite objects.

When applying the meta-language to lower-level abstractions, i.e. to rather more implementation-biased specifications, such a need may arise. The following notation is therefore offered. 


\section{Sub-References to TUPLE Elements}

Given:

(0)

$$
\text { dcl Tuple }:=\langle i .\rangle \text { type } D^{*}
$$

Retrieving the $i$ 'th component of eTuple is basically expressible as:

$$
\text { (c Tuple) [i] }
$$

with the selective update of that tuple position basically expressible as:

$$
\text { Tuple }:=\text { e Tuple }+[i \rightarrow a]
$$

It is now suggested to let:

$$
\text { Tupee: }[i]
$$

denote the reference to the $i$ 'th position of c Tuple, permitting (I) to be rewritten:

$$
\text { c(Tuple: }[i])
$$

and (2) as:

(2')

$$
\text { Tuple }[i]:=d
$$

\section{Sub-References to $M A P$ Range Elements}

\begin{tabular}{|c|c|}
\hline (1) & $(\subseteq \operatorname{Map})(a)$ \\
\hline (2) & $\underset{\sim}{\operatorname{MaR}}:=\subseteq \underset{\sim}{\operatorname{MaR}}+[a \rightarrow b]$ \\
\hline (3) & $\operatorname{Mag}(a)$ \\
\hline$(1 ')$ & $\underline{c}\left(\operatorname{Map}^{*}(a)\right)$ \\
\hline$\left(2^{\prime}\right)$ & $\operatorname{Map}_{\sim}^{*}(a):=b$ \\
\hline
\end{tabular}

The forms corresponding to $\left(1,2,3,1^{\prime}, 2^{\prime}\right)$ above, but for variables of type map:

$$
\text { dez Map }:=[\ldots] \text { type } A \underset{m}{D} \text {; }
$$

are: 
-- where a $\in$ dom $\subseteq$ Map prior to (2).

Sub-References to Sub-"TREE"s.

Given:

$$
D \quad:: s-n m_{1}: D_{1} s-n m_{2}: D_{2} \cdots s-n m_{2}: D_{l}
$$

and:

$$
\text { del Tree }:=m k-D(\ldots) \text { type } D \text {. }
$$

The forms corresponding to $\left(1,2,3,1^{\prime}, 2^{\prime}\right)$ above are now:

$$
s-n m_{i} \text { (ctree) } \quad 1 \leq i \leq 2
$$

$$
\begin{aligned}
& \text { (Let } m k-D\left(d_{1}, \ldots, d_{i}, \ldots, d_{l}\right):=c \text { Tree; } \\
& \text { Tree } \left.:=m k-D\left(d_{1}, \ldots, d_{i}, \ldots, d_{l}\right)\right)
\end{aligned}
$$

where all $d_{j}$ for $j \neq i$ are unchanged, $i . e$. where (2) only 'changes' the $s-n m_{i}$ sub-"tree". Now:

$$
s-n m_{i} \cdot \text { Tree }
$$

$$
\text { c(s-nmi "Thee) }
$$$$
s-n m_{i} \text { 'Thee }:=d_{i}^{\prime}
$$

\section{Discussion}

The reader may verify that a similar need for variables containing sets does not arise.

Also: the benefit of the derived reference operation, denoted by ' , is higher in forms (2') than (1') -- cf. forms (2) and (1), respectively.

Finally: the semantics of the derived reference operator, ', is fully explained by the equivalence of forms (1) and (1'), (2) and (2'), respectively. 


\section{STRUCTURED CLAUSES}

As used in this section a clause is either a statement or an expression.

It turns out that to each structured statement, and block, see next section, there corresponds a structured expression. The conditional form of such clauses syntactically looks very much alike, whereas this is not the case for the iterative clauses.

\subsection{Overview}

Let in the following $s$ and $S(\ldots)$ stand for statements, $E(\ldots)$ and suitably decorated e's for expressions, suitably decorated pe's for predicate expressions, and suitably decorated c's for clauses. It is further assumed that the c's of a given conditional structure are either all statements, or all expressions, leading to this conditional in turn being a statement, respectively an expression.

The following presents the various structured clauses in a schematic way:

\section{Condittional Cllauses}

(1) if pe then $c_{1}$ else $c_{2}$

(2) $\left(p e_{1} \rightarrow c_{1}\right.$,

$p e_{2} \rightarrow c_{2}$,

-..

$p e_{n} \rightarrow e_{n}^{\prime}$

(3)

$$
\begin{aligned}
\frac{\operatorname{cases}}{\left(e_{1}\right.}+e_{0}, \\
e_{2} \rightarrow c_{2}, \\
\cdots \\
\left.e_{n} \rightarrow c_{n}\right)
\end{aligned}
$$

$$
\text { and } \begin{aligned}
\left(2^{\prime}\right)\left(p e_{1}\right. & \rightarrow c_{1}, \\
p e_{2} & \rightarrow c_{2}, \\
\cdots & \\
T & \left.\rightarrow c_{n}\right)
\end{aligned}
$$

$$
\text { and (3') } \begin{aligned}
\frac{\text { cases }}{\left(e_{1}\right.} & \rightarrow a_{1}, \\
e_{2} & \rightarrow c_{2}, \\
\cdots & \\
T & \left.\rightarrow a_{n}\right)
\end{aligned}
$$




\section{Iterenative Clauses:}

(4) for $i=m$ to $n$ do $S(i)$

(4') $\quad<E(i) \quad \mid m \leq i \leq n>$

(5) for azz ideset do $S(i a)$ $\begin{cases}\left(5^{\prime}\right) & \{E(i d) \mid i d \in \text { set }\} \\ \left(5^{\prime \prime}\right) & {[i d \rightarrow E(\operatorname{map}(i d)) \mid i d \in \text { dom map }]}\end{cases}$

(6) white pe do $s$ $\left(6^{\circ}\right)\left\{\begin{aligned} & f(o b j)= \\ & \text { if } P(o b j) \\ & \text { then } F(o b j) \\ & \text { else } G(o b j, f(H(o b j)))\end{aligned}\right.$

where in the last schema ( $\left.\left(6^{\prime}\right)\right) P, F, G$ and $H$ are appropriate functions, $P$ being a predicate. $(4,5,6)$ are statements; $\left(4^{\prime}, 5^{\prime}, 5^{\prime \prime}\right)$ are expressions, and so is an application of $f$.

\section{Examples -- 53 :}

Illustrations of the duality between clauses (4) and (4'), respectively (5) and (5'), (5"), have already been given -- see sections 3.4, respectively $2.4,4.4$.

The duality of the conditional statements and conditional expressions need not be further discussed.

The following examples illustrate the duality of forms (6) and $\left(6^{\circ}\right)$.

To sum the elements of a tuple, $t$, of integers:

$$
\begin{aligned}
& \left(\underline{\operatorname{del}} q \quad:=t \underline{\text { type }} \operatorname{INTG^{*}}, \quad \operatorname{sum}(t)=\right. \\
& \text { sum }:=0 \text { type INTG; } \quad \text { if } t=< \\
& \text { while }(\underline{e} q) \neq<\underline{\text { do }}
\end{aligned}
$$

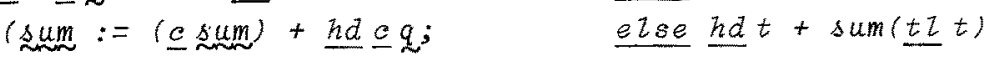

$$
\begin{aligned}
& q \quad:=\underline{t} \underline{c} q \\
& \text { return (c sum }) \text { ) }
\end{aligned}
$$

Subsequent elaboration functions will illustrate the power and neatness of recursive definitions centered around a simple if-then-else clause. 


\subsection{Detailed Syntax \& Semantics}

\subsubsection{If-then-else Conditional}

Schenga:

$$
\begin{aligned}
& \text { if expr } \\
& \text { then clause } \\
& \text { else clause }
\end{aligned}
$$

predicate-expression

consequent

alternative

\section{Meaning:}

Elaboration of an if-then-else clause proceeds as follows: first the premiss- (or test-) predicate expression is evaluated. If it does not yield a truth value an error has occurred -- and the meta-language program is in error! If the premiss yields truth then the consequent clause is elaborated. Otherwise the alternative clause. Any elaboration of either the consequent- or the alternative clause strictly succeeds the evaluation of the premiss.

\section{Pxogramming Notes:}

1. Observe that the form :

$$
\text { if expr then stmt }
$$

is not provided. Instead the programer, if so forced, is adviced to use:

$$
\text { if expr then stmt ezse I }
$$

for I see section 1.2 . Note that the converse is possible:

$$
\text { if } \sim \operatorname{expr} \text { then I else stmt }
$$

and that the 'similar' problem is not relevant for if-then-... expressions:

2. The premiss sometimes relates as follows to either the consequent or alternative clause: 
if $(\exists x \ldots)(P(x))$

then l let $x$ be s.t. $P(x)$; $e C(x))$

else $\ldots$

or, e.g.:

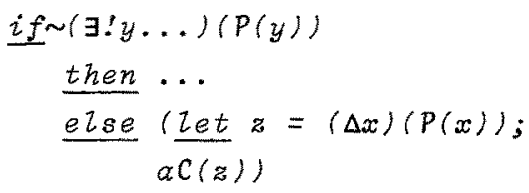

Whereever the 'connection' is so obvious, then we informally, but strictly speaking erroneously, write:

if $(\exists x \ldots)(P(x))$

then $c C(x)$

else...

respectively:

if $(\exists ! x \ldots)(P(x))$

then $\ldots$

else ac $(x)$

etcetera. The 'shifts' in identifier naming is, of course, immaterial. Thus the $x$ of the latter two premisses (informally) bind as far as the entire consequent and alternative clauses.

\subsubsection{McCarthy Conditional}

Schenema :

$$
\begin{aligned}
& \left(e_{1} \rightarrow e s_{1},\right. \\
& e_{2} \rightarrow e s_{2}, \\
& \cdots \\
& \left.e_{n} \rightarrow e s_{n}\right)
\end{aligned}
$$$$
n \leq 2
$$

and: 


$$
\begin{aligned}
& \left(e_{1} \rightarrow e s_{1},\right. \\
& e_{2} \rightarrow e s_{2}, \\
& \cdots \\
& \left.P \quad \rightarrow \quad e s_{n}\right)
\end{aligned}
$$$$
n \geq 2
$$

where the $T$ of the latter form is a meta-language keyword only used in McCarthy and Cases constructs in the $e_{n}$ 'position', i.e. in lieu of $\varepsilon_{n}$. All $e_{i}(1 \leq i \leq n)$ are predicate (or propositional) expressions.

\section{Meaning :}

We give the semantics of the above in terms of their transcription into the if-then-else form:

$$
\begin{aligned}
& \text { (if } e_{1} \text { then es } \\
& \text { else } \\
& \text { (if } e_{2} \text { then es }{ }_{2} \\
& \text { else } \\
& \text { ‥ } \\
& \text { else } \\
& \text { if } e_{n} \text { then } e s n \\
& \text { else error)....川 }
\end{aligned}
$$

respectively:

$$
\begin{aligned}
& \text { (if } e_{1} \text { then } e s_{1} \\
& \text { else } \\
& \text { (if } e_{2} \text { then } \\
& \text {.. } \\
& \text { else es }{ }_{n} \ldots 11
\end{aligned}
$$

\section{Programming Notee}

The same remarks concerning informal name binding and scope as for the if-then-else construct, apply with the constraint of the scope of an identifier defined in some $e_{i}(1 \leq i \leq n)$ restricted to the corresponding $e s_{i}:$ 


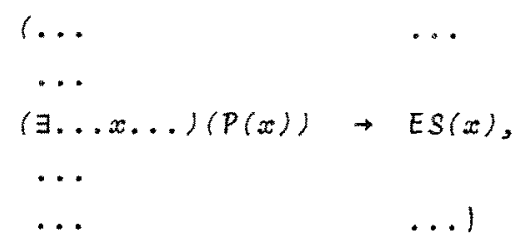

\subsubsection{The Cases Conditional}

\section{Schhema :}

$$
\begin{aligned}
\frac{\text { cases }}{e_{0}:} & \\
e_{2} & \rightarrow e s_{1}, \\
\cdots & \\
e_{n} & \left.\rightarrow e s_{n}\right)
\end{aligned}
$$

and:

$$
\begin{aligned}
\frac{\text { cases }}{\left(e_{1}\right.} e_{0}: & \\
e_{2} & \rightarrow e s_{1}, \\
\cdots & \\
T & \rightarrow e s_{2},
\end{aligned}
$$

with $T$ as defined above. The form of $e_{i}(1 \leq i \leq n)$ is either an ordinary expression, i.e. One all of whose free identifiers are bound by/in the surrounding context, or it is one of the so-called defining expression forms:

$$
\begin{aligned}
& \left\{d_{1}, d_{2}, \ldots, d_{k}\right\} \\
& \left.<d_{1}, d_{2}, \ldots, d_{k}\right\rangle \\
& m k-N m\left(d_{1}, d_{2}, \ldots, d_{k}\right) \\
& i a_{1}, \operatorname{cst}
\end{aligned}
$$

where the $d_{j}$ 's $(1 \leq j \leq k)$ are of either of these five forms. $i d$ represents identifiers, est constants.

\section{Programming Note:}

Usually the $d_{j}$ 's of an immediate defining expression are identifiers. 
Some of these may be bound in the containing scope. If all are bound or constants, then we have an ordinary expression.

\section{Meaning :}

We first assume ordinary, i.e. bound expressions, and explicate through reduction to earlier understood forms:

$$
\begin{aligned}
& \text { Let } v_{0}: e_{0} \text {; } \\
& \text { if } v_{0}=e_{1} \text { then es } \\
& \text { else } \\
& \text { (if } v_{0}=e_{2} \text { then }{ }^{e s} 2 \\
& \text { ezse } \\
& \text { 1 ... } \\
& \text { if } v_{0}=e_{n} \frac{\text { etse }}{\text { then }} \text { es } \\
& \text { e(se exrox)...1) }
\end{aligned}
$$

respectively:

$$
\begin{aligned}
& \text { Let } v_{0}: e_{0} \text {; } \\
& \text { if } v_{0}=e_{1} \text { then es } \\
& \text { else } \\
& \text { (if } v_{0}=e_{2} \text { then } e s_{2} \\
& \text { ezse } \\
& \text {... } \\
& \text { else es } n \text {...1) }
\end{aligned}
$$

\section{Name Binding \& Scoppe:}

We explain the free identifier, defining expression variants by specifically presenting an atypical combination and then transcribing it to an if-then-else form combined with let blocks. The example assumes all branch defining expressions identifiers free and rather casually mixes sets, tuples and trees! 


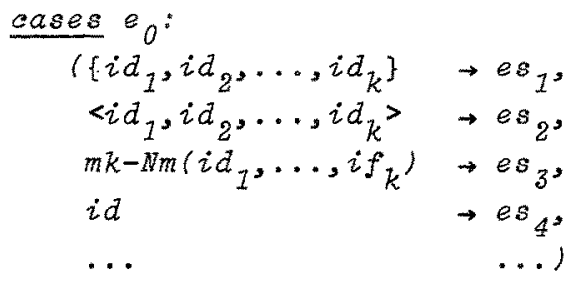

e.g. transcribes into:

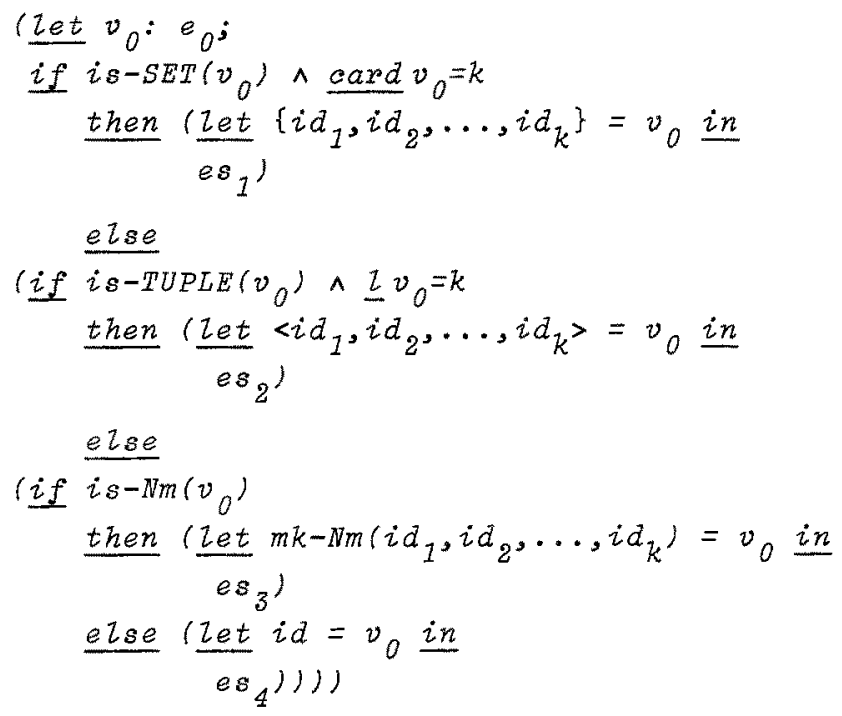

This latter form is now annotated (i.e. commented) referring alternatively to the former form:

First the base expression, $e_{0}$, is evaluated. The name $v_{0}$ identifies the evaluated object. Following the listing given in the cases form we now elaborate, in turn, successive branches until a match is found. Specifically: we first ask whether $v_{0}$ is a set and, if so, of cardinality $k$. If a fit is thus found we dissolve the set $v_{0}$ into its $k$ elements naming these $i d_{1}, i d_{2}, \ldots, i d_{k}$, whereupon the expression- or statement clause, es 1 , is elaborated. This terminates elaboration of the cases clause. If $v_{0}$ is not a set it is then asked whether it is a tuple, and if so, of length $k$. If a fit is thus found..., etc. If $v_{0}$ is not a set, tuple, nor a tree of type $N_{m}$ then $v_{0}$ is renamed $i d$ and $e s{ }_{A}$ is elaborated. Thus a free name, as here: $i d$, 'corresponds' to a $T$-clause -- leading to no elaboration ever of succeeding branches. 
The scope of the free identifiers of the branch-expressions is that of the corresponding expression, or statement, clause.

And so on: many variations, combinations and permutations (e.g. orderings of free- \& bound variables). The above 'schematic' examples have attempted to convey the general idea of defining expressions, their possible mixture of free- \& bound variables and even constants. The reader should, from this, be able to extrapolate. The basic point is this: since it is an abstraction, i.e. meta-language and since there is generally not to be an interpreter for it, anything sensible and context-wise obvious is to be allowed.

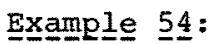

We shall use the meta-language conditional expressions and recursion to exemplify simpler versions of the conditional statements. That is: we applicatively define imperative constructs!

\section{A}

The syntactic domains:

$$
\begin{aligned}
& \text { Cond }=\text { If | MeC | Case } \\
& \text { If } \therefore \text { Expr stmt Stmt } \\
& \text { MCC } \quad \therefore \quad E x S t^{+} \text {[Stmt] } \\
& \text { Case :: Expr ExSt+ [Stmt] } \\
& \text { ExSt : : Expr Stmt }
\end{aligned}
$$

where Expr and Stmt are the meta-language expression and statement domains :

The semantic domains; first the textual, then the temporal:

$$
\begin{aligned}
& \rho \in E N V=I d \vec{m}(L O C \ldots) \\
& \sigma \xi \in S T G=L O C \vec{m} O B d
\end{aligned}
$$

where $I d$ is the syntactic domain of identifiers, LOC the further unanalyzed domain of locations. 


\section{Semantic Functions:}

Let $I$ and $V$ be names of the generic functions elaborating meta-language statements, respectively expressions, i.e.:

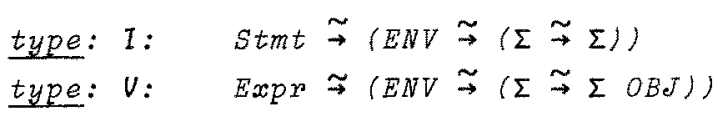




\subsubsection{The Ordered, Iterative For-To-Do Statement}

\section{Schhema:}

$$
\text { for } i d=\operatorname{expr}_{m} \text { to } \operatorname{expr}_{n} \text { do stmt }
$$

id must be free in the surrounding context. expr ${ }_{m}$ and expr ${ }_{n}$ must be integer valued expressions. All occurrences of $i d$ in stmt are free in stmt.

\section{Programming Note}

We usually constrain expr ${ }_{m}$ and $\operatorname{expr}_{n}$ to be so-called static expressions. These are expressions whose value can be ascertained statically, without reference to any computation (e.g. state). As such, static expressions are usually either constants (viz.: expr ${ }_{m}=1$ ) or simple operator/operand expressions involving only textual, i.e. syntactic domain objects.

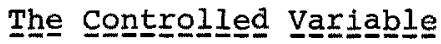

id is called $a$, or the, controlled variable. It is not a variable in the sense of denoting a REFerence, and thus it cannot be changed in stmt. $i d$ denotes an integer.

\section{Name Binding \& Scope:}

The first occurrence of $i d$ in "for $i d=\ldots$ " is the defining occurrence. It binds all (free) occurrences of id in stmt. The scope of this controlled variable is the for statement in which it is defined.

\section{Meaning:}

We first explicate the primarily intended usages of the for statement: namely the ones which have $\operatorname{expr}_{m}$ and $\operatorname{expr}_{n}$ being static expressions evaluating to $m$, respectively $n$. Let $s(i d)$ be another way of alluding to stmt. Then:

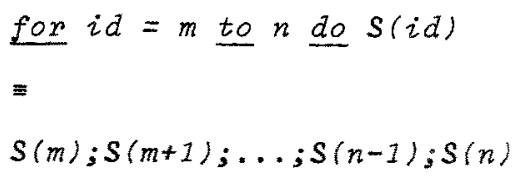


Thus if $m<n$ then: "for $i d=m$ to $n$ do $S(i d)=" I "$. Given $S(i d), S(k)$ means the substitution of all id in $s$ by $k$.

We then explain the meaning of for statement whose from and to expressions, $\operatorname{expr}_{m} \& \operatorname{expr}_{n}$ are expressions of any kind. To this end we apply the meta-language itself!

\section{Example 5 5 :}

Let the for statement syntactic domain specification be:

$$
\text { For } \quad: \quad \text { Id Expr Expr Stmt }
$$

where IdCTOKEN, and Expr \& Stmt denote the class of respectively sourcelanguage expressions and statements.

Let the semantic domains pertaining to the interpretation of For statements be the temporal store:

$$
\sigma \in S T G=L O C \vec{m}^{O B J}
$$

which maps LOCations to OBJects, and the simplified textual environment:

$$
\rho \in E N V \quad=\quad I d \vec{m}(L O C|I N T G| \ldots)
$$

which maps source-language text Identifiers into either Locations, if they are names of declared variables, or to INTeGers, if they are e.g. controlled variable names, or... .

Let $V$ denote the generic expression evaluation function which applies to source -language expressions, environments \& states/stores and yields state changes \& objects -- in this case INTeGers. I denotes the generic statement interpretation which applies to source-language statements, en vironments \& states/stores and yields state changes. 


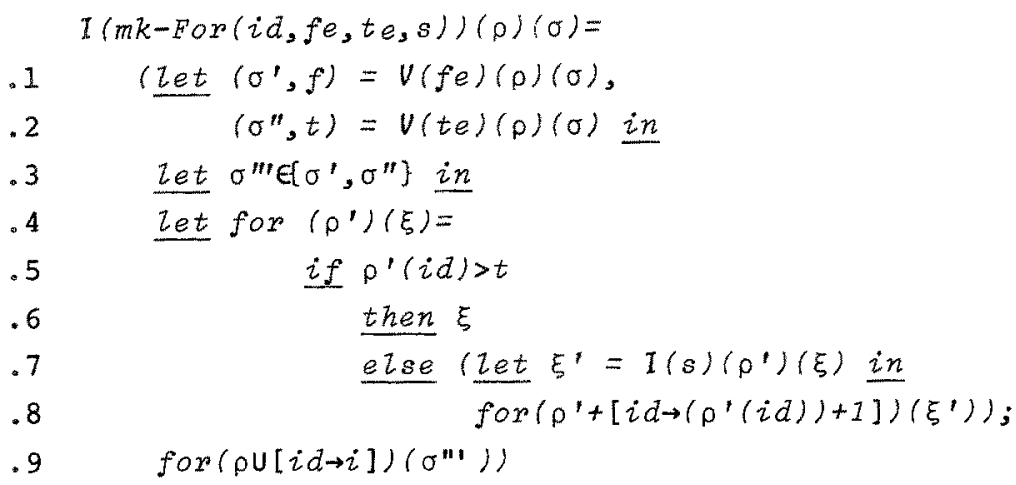

\section{Annotations :}

.1-.2 Both specification expressions, the from expression and the to expression, are evaluated in parallel "," (or in any order), and both may lead to implicit state changes $\left(\sigma^{\prime}\right.$, respectively $\sigma^{\prime)}$ and to the explicitly desired from, $f$, and to, $t$, values.

.3 This line illustrates the problem: which new (or next) state, $\sigma^{\prime}$ or $\sigma^{\prime \prime}$, to choose. In this case the problem has been brought upon us by our insistance on parallel evaluation of all specification expressions. Had our pragmatics lead us to choose sequentiality of from- and to expressions evaluation there would have been no need for a non-deterministic choice.

.4 A local function, for, is recursively (.4 vs. .8) defined which if applied:

will test whether the controlled variable value $\left(\rho^{\prime}(i d)\right)$ has gone beyond the limit $(t)$,

.6 if so, the final state is the one current when the last invocation (.8 or .9) of for was made, i.e. at the end of last iteration, respectively when for was first called (initialized);

.7 otherwise the value of the controlled variable is still within bounds, and the 'body', $s$, of the for statement shall be Interpreted, leading to a new state, $\xi^{\prime}$.

.8 With this state, and the 'updated' environment which binds the (textual) name of the controlled variable to its incremented value the for loop is invoked (recursively). 
This completes the definition of the auxiliary for function,

.9 which is first activated with the 'initial' state, $\sigma$ "', and an environment which binds the controlled variable to its initjal value, $i$.

10 Note that the environments are simply extended versions of the environment in which the for statement is interpreted.

In summary: the meta-language for loop was conceived of as a means for iterating through syntactic tuple structures, $t l$. Hence the standard requirement that $\operatorname{expx}_{m}$ and $e x p r_{n}$ be static expressions, the former usually 1 , the latter usually $\underline{I} t$.

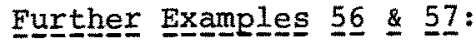

We now illustrate imperative and applicative definitions of the Compound Statement source language construct:

Cmpd :: stmt*

The imperative version is basically based on a state:

$$
\begin{aligned}
& \Sigma_{i}=(\underline{S T G} \rightarrow S T G) \underline{U} \ldots \\
& \text { Int-Cmpd (mk-Cmpd(stl))(p)= } \\
& \text { for } i=1 \text { to } z \text { st } \text { do int-Stmt (st }[\text { [i])( } \rho) \\
& \text { type: Cmpd } \simeq\left(E N V_{i} \sim\left(\Sigma_{i} \underset{\rightarrow}{\rightarrow} \Sigma_{i}\right)\right)
\end{aligned}
$$

The applicative version is similarly based on a state:

$$
\begin{aligned}
& \Sigma_{a} \quad:: S T G \ldots \\
& I-C m p d(m k-C m p d(s t z))(\rho)(\sigma)= \\
& \text { if } s t l=<> \\
& \text { then } \\
& \text { else }\left(\underline{\text { Zet }} \sigma^{\prime}=I-S t m t(\underline{h} \operatorname{st} \text { L) }(\rho)(\sigma) \underline{i n}\right. \\
& \left.I-C m p d(m k-C m p d(\underline{t} s t \tau))(\rho)\left(\sigma^{\prime}\right)\right) \\
& \text { type: Cmpd } \stackrel{\sim}{\rightarrow}\left(E N V_{a} \stackrel{\sim}{\rightarrow}\left(\Sigma_{a} \sim \Sigma_{a}\right)\right)
\end{aligned}
$$


where correspondingly:

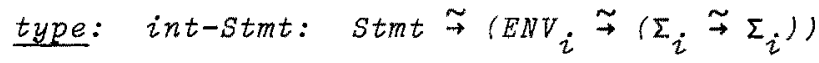

$$
\begin{aligned}
& \text { type: I-Stmt: Stmt } \underset{\sim}{\sim}\left(E N V_{a} \sim\left(\Sigma_{a} \sim \Sigma_{a}\right)\right)
\end{aligned}
$$

\subsubsection{The unordered For-A11 \& Parallel Statements}

The For-All statement

\section{Scherma:}

for all def $\in$ set do stmt

where def and stmt pairwise are of the forms:

$$
\begin{aligned}
& i d \\
& \left\{c d_{1}, c d_{2}, \ldots, c d_{n}\right\} \\
& \operatorname{cod} d_{1}, c d_{2}, \ldots, c d_{n}> \\
& m k-N m\left(c d_{1}, \ldots, c d_{n}\right)
\end{aligned}
$$

$\sim s\left(i d_{1}, i \bar{d}_{2}, \ldots, i d_{m}\right)$

where a proper subset of $c d_{1}, c d_{2}, \ldots, c d_{n_{1}}$ may be constants, otherwise of either of the above four listed forms, usually identifiers, $i d$ !

These must all be distinct. And where $i d_{1}, i d_{2}, \ldots, i d_{m}$ are the identifiers of def free in the containing context. All such must be free in $S$. It must be statically decidable that set denotes a set. And if either of the latter three def forms are used, then it must be statically decidable that set is of a logical type, $y$, such that either some $X-s e t, X^{*}$ or $N m$ is contained in $Y$; viz.: $X-\operatorname{set} \subseteq Y, X^{*} \subseteq Y$ or: $N m \subseteq Y$ !

\section{The Controlled Variables}

The identifiers of def free in the containing context are the controlled variables. Again, as in section 9.2.4 they are not assignable but directly denotes non-REFerence OBJects.

\section{Name Binding \& Scope}

The identifier occurrences in def free in the containing context are defining occurrences. They bind all their (free) occurrences in Stmt ( $S$ ). The scope of these controlled variables is the for statement in which they are defined. 


\section{Meaniㅗㅁㅗ :}

In this section we only explicate the semantics of the for-all statement for the simple case of static set expressions and simple identifier def's -- this is also the most common case. Let set denote a set of $k$ objects, and name these $i d_{1}, i d_{2}, \ldots, i d_{k}$. That is set $\sim\left\{i d_{1}, i d_{2}\right.$, $\left.\ldots, i d_{k}\right\}$ :

for $a l z i d \in\left\{i d_{1}, i d_{2}, \ldots, i d_{k}\right\}$ do $s(i d)$

$\sim$

$/ /\left\{s\left(i a_{1}\right), s\left(i a_{2}\right), \ldots, s\left(i d_{k}\right)\right\}$

\section{The Parallel Statement}

\section{Scheme :}

$$
/ /\{\operatorname{stm} t, \operatorname{stmt2}, \ldots, \operatorname{stmtn}\} \quad n \geq 2
$$

\section{Meaning:}

Elaboration of the statements stmt1, stmt2, ..., stmt $n$ proceeds in parailel, "independently" of each other. Elaboration of this collateral clause terminates as a result of all statements having been elaborated.

\section{Examples 5묘- $\underline{5}$ :}

We now illustrate the semantics of a source language statement similar to the parallel statement. Instead of elaborating the statements in parallel, they are just executed in any, arbitrary order:

$$
\text { Alt :: stmt-set }
$$

is the syntactic domain specification. The definition is kept applicative 


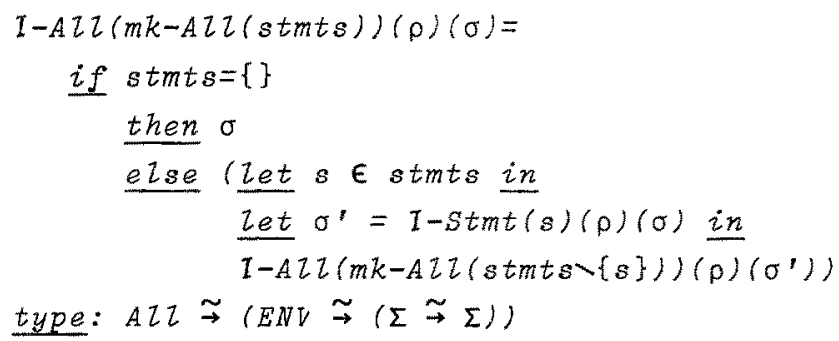

A for-all source language construct could be given the following definition:

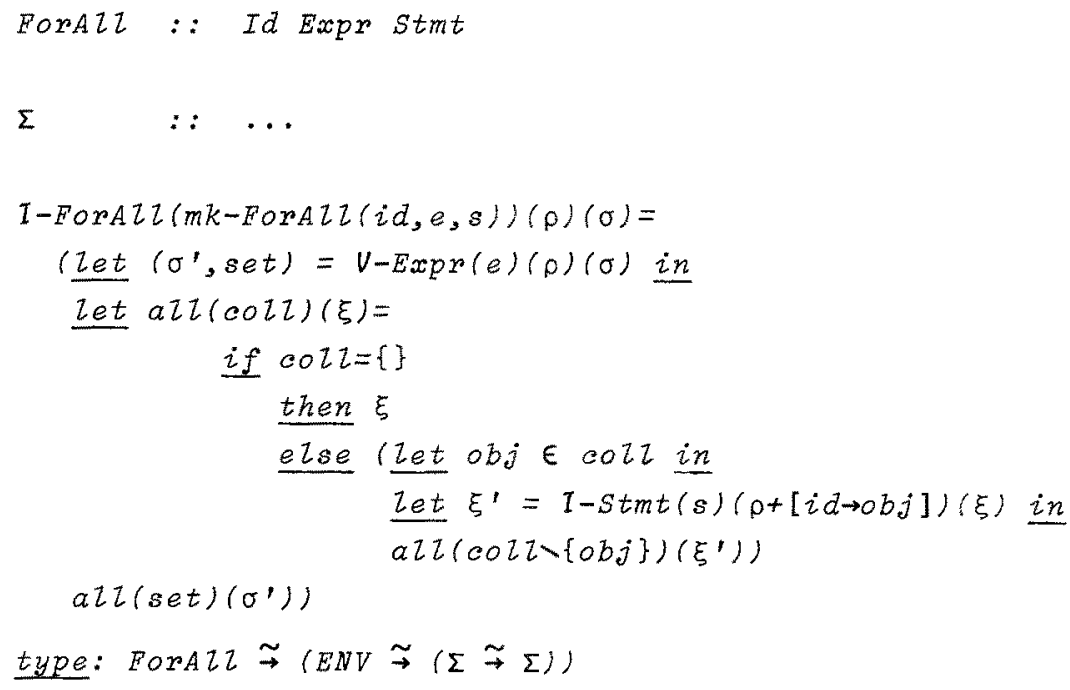

where it is assumed that the evaluation function, which elaborates $i d$, finds the meaning of $i d$ just by looking up in the environment:

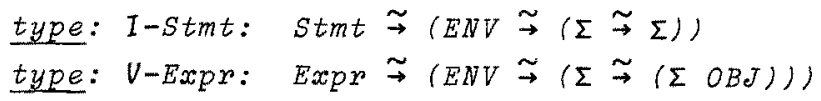

\subsubsection{The While-Do statement}

\section{Schema :}

while expr do stmt

where it is statically decidable that expr is a truth-valued expression. 


\section{Programming Note:}

expr is usually an impure expression, i.e. one whose value depends on the current state. The state is, of course, potentially being changed by $s$ tmt.

\section{Meaning :}

As you might expect it -- but here is an applicatively expressed forma lization of the imperative while construct.

\section{Example 므:}

Abstract Syntax:

Wh $\quad:$ Expr Stmt

for the syntactic domain. And:

$\sigma \in S T G=L O C \vec{m}$ OBJ
$\rho \in E N V=I d \vec{m}(L O C \mid \ldots)$

for the semantic domains.

\section{Semantic Functínons:}

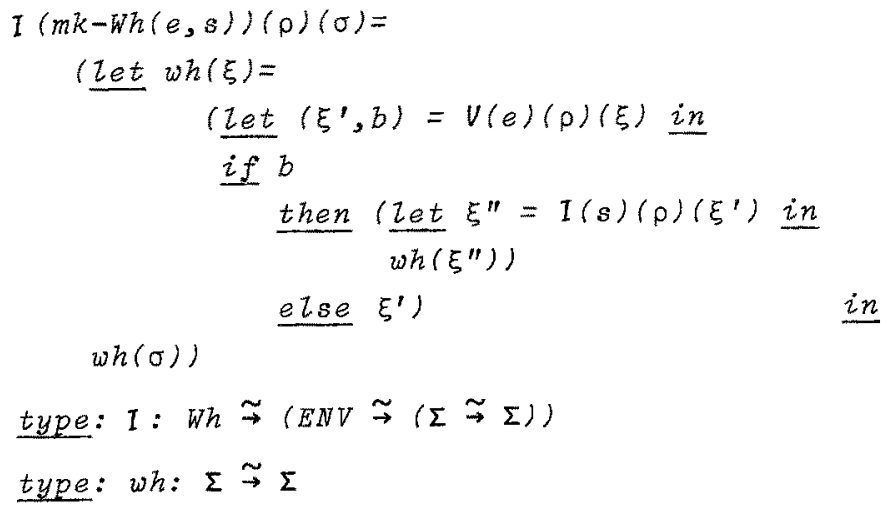




\section{Example 61:}

Dijkstra's Guarded, Repetitive Construct [Dijkstra 75]:

$r g \in$ RepGuard :: (Expr Stmt)-set

with STG and ENV as above, ilas the following 'informal' semantics: Execute rg as long as evaluation of at least one expression in the un= ordered set of pairs of Expression-Statements yields true:

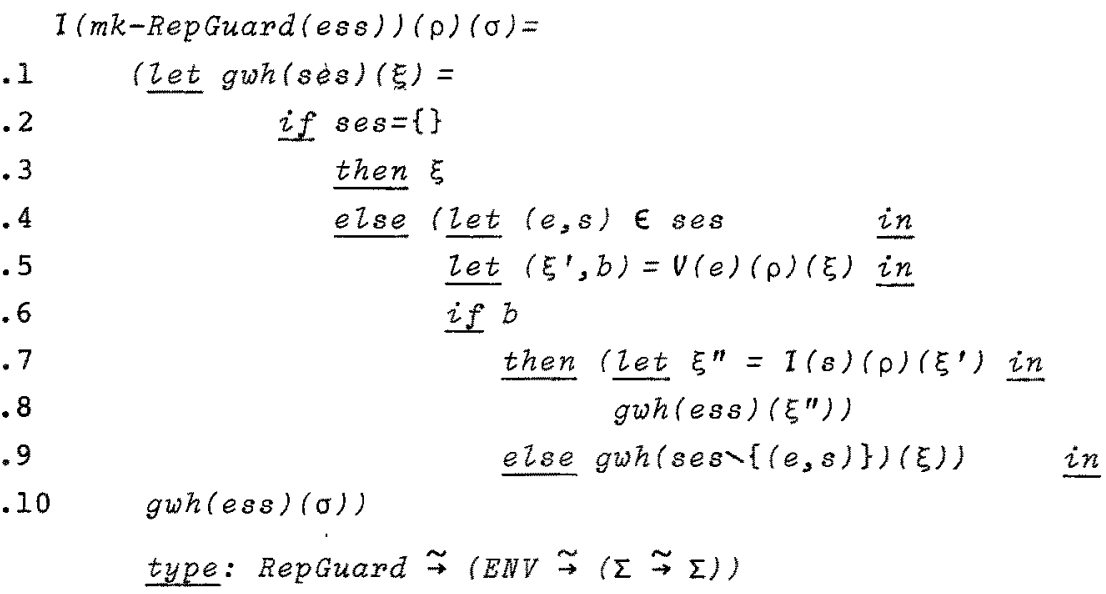

In this definition, evaluation of guards (e) may cause side-effects, i.e.: $\xi$ goes to $\xi^{\prime}$. Thus repeated evaluations of some (one) guard, in= itially yielding falsity, might eventually produce truth -- had we not passed the initial state $(\sigma$, subsequently $\xi$ ) onto alternative evalua= tions (.9). Doing so also secures Dijkstra's requirement that if some guard is chosen, then it was true in the 'initial' state ( $\sigma$, subse= quently $\xi$ ).

The non-deterministic aspect of this construct is exhibited by the ar= bitrary set element selection of line .4.

The reader may verify that this command is a (sledge-hammer?) general= ization of the whize do construct above! 


\section{BLOCKS}

A compound statement:

$$
\left(\operatorname{stmt}_{1} ; \operatorname{stmt}_{2} ; \cdots ; \operatorname{stm}_{n}\right)
$$

is a sequence of two or more statements. The semicolon, "; , acts as the combinator here.

A block:

$$
\begin{aligned}
& \text { (Iet } \left.i d=\operatorname{expr}_{d} \underline{i n} \operatorname{expr}_{b}\right) \quad \text { (Let } i d=\operatorname{expr} \text { in stmt) } \\
& \text { (Let id: } \operatorname{expr}_{d} \text { jexpro } p_{b} \quad \text { (Let } i d: \text { expr; stmt) } \\
& \left.\left(\underline{d c l} \text { vr }:=\operatorname{expr}_{d} ; \operatorname{expr}_{b}\right) \quad \text { ( } \underline{d c l} \text { vr }:=\operatorname{expr} ; s t m t\right)
\end{aligned}
$$

consists of a (Let) definition of a constant, non-peference object, or a (del) declaration (of a variable) -- on one hand --, compounded with an expression, or a statement (-sequence) -- on the other hand. The com= binators here are either the semicolon, ";", or the "in" symbol.

This section will not bring very many examples. Previous sections abound with examples of blocks. We leave it to the reader to look these up for re-confirmation:

\section{Reference to blockk-examples:}

Parenthesized numerals refer to example numbers, the $i . j-k$ trailing these refer to formulae (i) and line number $(j-k)$ sequences.

Applicative expression blocks:

(0) 11.3-8, (0) 11.6-8, (0) 16.1-6, (0) 17.3-7, (0) 18.3-21, (0) $18.8-21$, (0) $20.3-11, \ldots$

Imperative expression blocks:

$$
\begin{aligned}
& \text { (31) } 14.3-6,(31) 14.12-17, \text { (50) } 2.0-3, \text { (50) } 3.0-3, \text { (52) } 4.3-7 \text {, } \\
& \text { (52) } 5.1-4, \ldots
\end{aligned}
$$

Statement blocks:

(31) $13.1-8$, (52) $1.3-8$, (52) $2.1-2$, (52) $3.1-3, \ldots$ 


\section{The Block Concept}

The block concept of the meta-language essentially deals with the notion of scope. Blocks are of the forms:

$$
(\text { Let } i d=\operatorname{expr} \text { in }
$$

and:

$$
\text { (Let id: expr; }
$$

We have, however, in past examples, also permitted ourselves to introduce local variables:

$$
\left(\frac{\text { del } i d}{\text { clause). }}:=\operatorname{expr} \text { type } D ;\right.
$$

In all three cases $i d$ stands for a locally, i.e. a block, defined, constant quantity. We say that the scope of $i d$ is the block: (...) in which it is defined. Only in this textual part, may it be referred to. In the first two blocks $i d$ denotes the value of expr upon block entry. In the last case $i d$ denotes the location of a meta-storage cell, i.e. an object in the domain of $\Sigma$ :

$$
\Sigma=\cdots \underline{U}(I d \underset{m}{m} D)
$$

In particular:

$$
\sigma=\ldots \cup[i d \rightarrow o b j]
$$

where $i d$ is the denotation of $i d$, and $o b j$ is the value of expr upon block entry.

The let (or del) definitions of $i d$ are said to bind any free occurrences of $i d$ in clause. Thus the scope of id extends to inner, nested blocks in which $i d$ is not redefined.

clause may be a meta-language expression or a sequence of one or more statements. 


\subsection{Let Constructs}

Observe the two distinct cases of let constructs:

The syntactic, or applicative let construct:

$$
\text { (Let } i d=\operatorname{expr} \text { in }
$$

If expr is a pure expressien, i.e. an expression whose evaluation does not require access to any state components, then we use " $=$ " and "in" as delimiters.

The semantic , or imperative let construct:

$$
\text { (let id : expr; }
$$

If, on the other hand, evaluation of expr requires access to the state, i.e. if expr is an imperative, or impure, expression, then we use ":" and ";" as delimiters.

Assume $i d$ to occur free in clauce, i.e. think of clause as $c(i d)$. Then the meaning of the syntactic let clause block is $c(e x p r), i . e$. can be obtained by substituting expr for all free occurrences of id in clause. Note that the substitution could be done on the syntactic level, i.e. by replacing texts. The meaning, however, of the semantic let clause block is (val), where val is the value of expr upon block entry. That is: the meaning can be obtained by substituting velues of expr for all free occurrences of $i d$ in clause.

\section{Let Construct Variants}

A very useful let variant is:

$$
\frac{(\text { Let }}{\text { clause) }} \in \text { b } \underline{b e} \underline{s} \cdot \underline{t} \cdot P(o b j) ;
$$


which we understand as: let $o b j$ be an object in the domain or set $D$ such that it satisfies the predicate $P$. Other variants, which are simple cases of the above are:

$$
\text { (Zet obj } \in \text { set; }
$$

and:

$$
\text { ( Let obj be s.t.t. P }(o b j) \text {; }
$$

The above three variants were indicated, by the ";" separating the let construct from the block body clause, to be of the semantic type. Similar, but syntactic constructs would instead of the infix ";" use an infix "in".

\section{Composite Object Let Decomposition}

The following are useful, so-called composite let constructs:

$$
\begin{aligned}
& \left(\frac{\text { Let }}{\text { clause }} m k-D\left(d_{1}, d_{2}, \ldots, d_{n}\right)=\operatorname{expr}_{d} \frac{i n}{}\right. \\
& \left(\frac{\text { Let }}{\text { clause }}<d_{1}, d_{2}, \ldots, d_{n}>=\operatorname{expr}_{t}\right. \text { in }
\end{aligned}
$$

and:

$$
\left(\operatorname{let}_{\text {et }}\left\{d_{1}, d_{2}, \ldots, d_{n}\right\}=\operatorname{expr}_{s}\right. \text { in }
$$

Their respective meanings are:

exprast evaluate to a $D$ tree object, with:

$$
D^{\prime} \quad \therefore D_{1} D_{2} \ldots D_{n}
$$

then the above is equivalent to: 


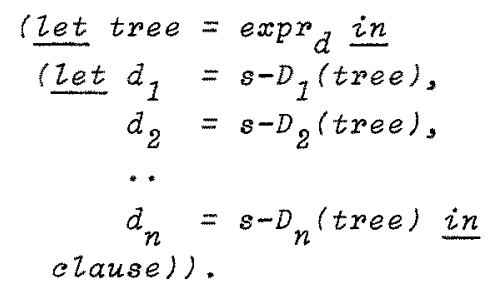

$\operatorname{expr}_{t}$ must evaluate to an $n$-tuple:

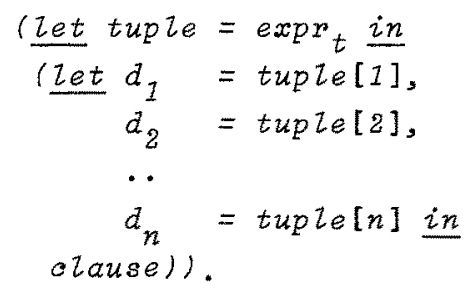

$\operatorname{expr}_{s}$ must evaluate to a set of cardinality $n$. The 'assignment' of set objects to $d_{i}$ is arbitrary:

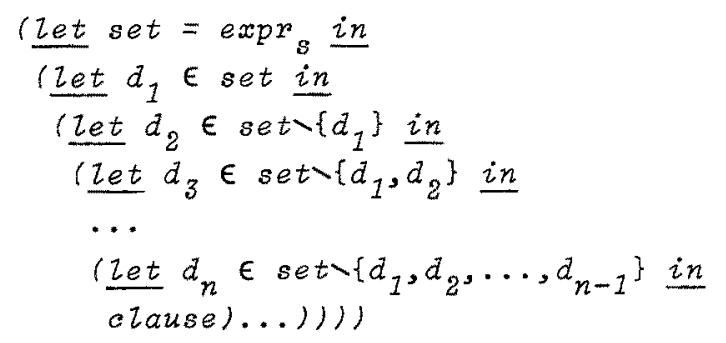

\section{$\underline{\text { Simultaneous \& Recursive Let Definitions }}$}

The form:

$$
\begin{aligned}
\text { (ret } a_{1} & =e_{1}, \\
d_{2} & =e_{2} \\
\cdots & \\
a_{n} & =e_{n} \text { in } \\
\text { clause) } &
\end{aligned}
$$

simultaneously defines the objects $d_{1}, d_{2}, \ldots, d_{n}$. They are, in general, to be the ("smallest" such) objects which satisfy the (above set of simultaneous) equations. This description permits $d_{j}$ 's to occur recursively in the equation set. The $d_{j}$ 's might be composite constructs, 
introduced in the previous subsection, in which case we mean that the constituent (free) identifiers (ultimately occurring in $d_{j}$ ) may occur recursively in the equation set.

For the story on recursive definitions of functions we refer to section 6.2 .

\section{Notational Conventions}

The form:

$$
\begin{aligned}
& \text { (let } d_{1}=e_{1} \frac{i n}{\text { let }} d_{2}=e_{2} \text { in } \\
& \text { let } d_{n}=e_{n} \text { in } \\
& \text { clause) }
\end{aligned}
$$

is a short-hand for:

$$
\begin{aligned}
& \text { (Let } d_{1}=e_{1} \frac{i n}{\left(\text { Let } d_{2}\right.}=e_{2} \text { in } \\
& (\ldots) \\
& \left(\frac{\text { Let }}{\text { (Lause }) . . .)) ~}\right.
\end{aligned}
$$

The form :

$$
\text { ( Let } m k-A(, c,)=e_{a} \text { in }
$$

where e.g.:

$$
A \quad: \quad B \quad C \quad D \text {, }
$$

is a short-hand for:

$$
\operatorname{liet}_{\text {clause }} m k-A(b, c, d)=e_{a} \underline{i n}
$$


It is used to alert the reader to the non-use of the $B$ and $D$ components of the $A$ tree denoted by $e_{a}$.

Similarly for anonymous trees; and:

$$
\frac{(\text { let }}{\text { clause }}<x_{,}, z,>=e_{t} \underline{i n}
$$

which is the same as:

$$
\begin{aligned}
& \text { (Zet } t=e_{t} \text { in } \\
& \text { Zet } x=t[1], \\
& z=t[3] \text { in } \\
& \text { clause). }
\end{aligned}
$$

\subsection{Pure \& Impure Expressions}

A pure (or applicative) expression is one whose evaluation never requires access to the state.

An impure (or imperative) expression is one whose evaluation potentially requires access to the state.

Given that $\Sigma_{i}$ denotes the state space of an imperative (global variable only) model we can say that the type of the denotation of an impure expression is either of the form:

$$
\Sigma_{i} \stackrel{\sim}{\rightarrow}\left(\Sigma_{i} \text { OBJ }\right)
$$

or of the form:

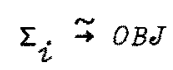

The formex type hints that evaluation of the meta-language expression potentially leads to a (side-effect) state-change, whereas the latter type only expresses that the state is accessed in order to compose the resulting $O B J$ ect value. 
The choice of forming an expression either as a pure-, or as an impure, expression, is solely determined by the kind of object to be denoted. That is: even though some abstract model is primarily centered around global state variables, some objects may still be denotable by pure expressions.

If at least one target expressions of a conditional expression is impure, then all such target expressions are to be impure. This requirement is further motivated in [Jones 1978a]. To render an otherwise pure expression impure prefix it with the operator return. See section 10.6 .

\subsection{The ";" Combinator}

The ";" is a combinator. Its use can be explained in two ways: syntactically, and semantically. Syntactically speaking, "; separates imperative clauses: statements; semantic let clauses; a semantic let clause from a statement; and a statement from an impure expression:

$$
\begin{aligned}
& \left(\ldots \text { stmt }_{1} ;\right. \text { stmt } \\
& (\ldots) \\
& \left(\ldots \text { let } x_{1}: \operatorname{expr}_{1} ; \text { let } x_{2}: \operatorname{expr}{ }_{2} ; \ldots\right) \\
& (\ldots \text { let } x: \operatorname{expr} ; \text { iexpr) } \\
& (\ldots \text { stmt; iexpr) }
\end{aligned}
$$

Semantically speaking ";" denotes functional composition. Since the type of the denotation of a meta-language semantic let clause, let, or statement, stmt, is:

$$
\text { let, stmt: } \Sigma_{i} \stackrel{\sim}{\rightarrow} \Sigma_{i}
$$

The construct:

$$
(c 1 ; 02)
$$

where (c1,c2) are either (semantic lets, statements) or (statements, statements), means:

$$
\lambda \sigma \cdot(c 2(c)(\sigma)))
$$

where $c i$ is the meaning of $c i(i=1,2)$. Thus: 
$\begin{array}{lll} & : & \sim \lambda c 1 . \lambda c 2 . \lambda \sigma .(c 2(c 1(\sigma))) \\ \text { i.e. } & ; \quad \varepsilon \quad(\Sigma \cong \Sigma) \rightarrow((\Sigma \cong \Sigma) \rightarrow(\Sigma \sim \Sigma))\end{array}$

\subsection{Compound Statements \& Statement Sequences}

The meta-language permits any statement to be a compound statement:

$$
\left(\operatorname{stm}_{1} ; s t m t_{2} ; \cdots ; \operatorname{stm}_{n}\right)
$$

The body of a (statement-) block, i.e. the syntactic construct referred to as clause in e.g. section 10.1 , may be a statement sequence:

$$
\operatorname{stmt}_{1} ; \operatorname{stmt}_{2} ; \ldots ; \operatorname{stm}_{n}
$$

There is no semantic difference between these two constructs. We omit parenthesizing the latter since it is always superfluous.

The formal meaning is:

$$
\left.\lambda \sigma \cdot \operatorname{stm} t_{n}\left(\operatorname{stm} t_{n-1}(\ldots)\left(s t m t_{2}\left(\operatorname{stm} t_{1}(\sigma)\right)\right) \ldots\right)\right)
$$

where $\operatorname{stm} t_{i}$ is the meaning of $s t_{i}{ }$

We refer to [Jones 1978a] for a further $\lambda$-definition of the meanings of the basic statements.

The informal meaning is as you would expect it to be.

\subsection{Statement- \& Expression Blocks}

A statement-block is a statement. The block, when interpreted, effects

a state-change. An expression-block is an expression. The block, when evaluated, may effect a state-change, but always, in addition, delivers a value:

$$
\begin{array}{ll}
\text { stmt-block: } & \Sigma_{i} \sim \Sigma_{i} \\
\text { expr-block: } & \Sigma_{i} \cong\left(\Sigma_{i} \text { OBJ }\right)
\end{array}
$$

A statement block consists of a sequence of one or more syntactic and/or 
semantic $\underline{\text { let }}$ clauses followed by a sequence of one or more statements.

An expression block is either a pure- or an impure- (i.e, an applicative-, respectively an imperative-) expression. A pure expression block consists of one or more syntactic let clauses followed by a pure expression. An impure expression block consists of a non-zero length sequence of zero, one or more syntactic- or semantic let clauses followed either by an impure expression or a statement all of whose syntactically possible execution paths end with an impure expression. Since only wellstructured statements are permitted the test for this latter is quite simple.

\subsection{Return}

From the explanation of ";" it follows that if a statement sequence, of an expression block, is to be followed by an expression, then the type of this expression must be:

$$
\Sigma \rightarrow(\Sigma O B J)
$$

i.e. impure.

In general, if the context determines that an expression be impure, and the value to be yielded can be denoted by a pure expression, $e$, then we need to render e impure. This is the purpose of the monadic expression operator return.

$$
\begin{aligned}
& \text { return } \sim \lambda o b j \cdot \lambda \sigma \cdot(\sigma, 0 b j) \\
& \underline{\text { return }} \in(O B J \rightarrow(\Sigma \rightarrow(\Sigma O B J)))
\end{aligned}
$$

\section{Examples}

See examples: (31) 14.6, (31) $14.17,(50) 2.3,(50) 3.3,(50) 4.5$. 
11. EXITS

Even though the meta-language has imperative constructs, it lacks the conventional GOTO construct. Hence it lacks labels.

The exit mechanisms, in many ways, replace the GOTO construct. You may say, without being grossly wrong, that exit provides a strructúured GOTO, albeit, in general, to a dynamically determined, and -- in any case -unlabelled, program point.

\section{Example $\underline{6}$ :}

We use the exit mechanism when modeling the GOTO concept of our running example source language: Therefore: in the following read carefully. observe the distinction between source- and meta-language constructs, in particular the block constructs.

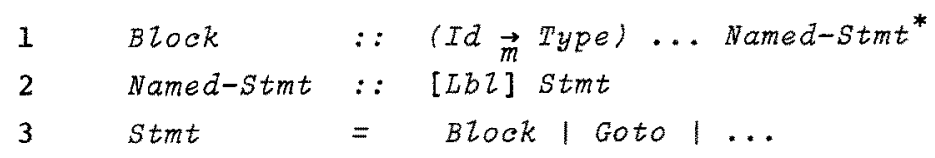

In this source language Gotos may not go into phrase structures. That is: Gotos may e.g. go from one statement of a Named-statement list to another statement of the same list, or out of the containing Block to a statement of the Named-Statement list of the next embracing Brock, in fact it may go out of any such number of nested levels. Each time a Block activation is left, whether through (normal) epilogue due to all statements having been executed, or whether due to a global Goto, the same 'clean-up' epilogue actions -- as were illustrated in e.g. example 31, section 4.5 -must first take place:

4 int-Block $(m k-B$ Lock $(d c l s, p r o c s, n s t z))(\rho, c a)=$

.1 (Let aid E AID be s.t. aid ne ca in

.2 Let $\rho^{\prime}: \rho+[2 b l \rightarrow m k-L A B(a i d, 2 b l) \mid$ lbl $\in$ Labels(nstl) ]

$.3+[v \rightarrow \operatorname{get-Zoc}(\operatorname{dez} s(v))(\rho) \mid v \in \underline{\operatorname{dom} d e l s}]$

$.4+\left[s-I D(p) \rightarrow \operatorname{eval-Proc}(p)\left(\rho^{\prime}\right) \mid p \in\right.$ procs $]$;

.5 always

.6 (Zet Loes $=\left\{\rho^{\prime}(v) \mid v \in\right.$ domdeis $\}$ in

.7 let slocs $=\{\ldots$ see example $31 \ldots\}$ in

.8 STG $:=$ esTG stocs)

.9 in int-Named-Stmt-list(nstz)(p,cau\{aid\}, aid))

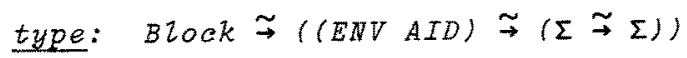


.4 eval-Proc is the imperative version of example 40 's V-Prc, as used in example 42 (section 6 , respectively 6.3 ).

.2 The denotation of a label is in the domain:

$$
\angle A B:: A I D L B Z
$$

The AID component serves to keep track of active source language block activations.

- I The prologue action of a Block selects an activation identifier not in the set of (identifiers selected by all) current activations.

.2 The block environment associates all Labels of its named statement list with this (unique) activation (identifier).

. 4 Since procedures may be recursive, and -- independently thereof -since gotos may occur from within a procedure (activation) to the named statement lists of any of its embracing, including defining, blocks, the environment passed to eval-Proc is the environment being (thus recursively) defined.

We shall return to lines $.5-, 9$, which -- on the whole -- resemble lines .4-.8 \& .3 of int-Block of example 31, section 4.5 .

5 int-Named-Stmt-Zist(nstz) $(p, c a, a i d)=$

.1 (tixe $[m k-L A B(a i d, z) \rightarrow$ cue-int-Named-Stmt-iist $(i, n s t z)(\rho, c a)$

$.2 \quad \mid 1 \leq i \leq$ Len nsti $\wedge s-L b i$ (nsti[i] $=i \neq n i z]$

.3 in cue-int-Named-Stmt-iist $(1, n s t l)(\rho, c a))$

6 cue-int-Named-Stmt-iist $(i, n s t i)(p, c a))=$

.1 for $j=i$ to zen $n s t z$ do int-Stmt $(s-\operatorname{stmt}(n s t z[j]))(\rho, c \alpha)$

7 int-Stmt $(\operatorname{stm} t)(\rho, c a)=$

.1 cases stmt:

$.2(m k-G o t o(Z b l) \rightarrow \operatorname{exit}(\rho(z b l))$,

$.3 \quad m k-B \operatorname{lock}(\ldots) \rightarrow \operatorname{int}-B \operatorname{lock}(\operatorname{stm} t)(\rho, c a)$,

... $\quad \ldots$

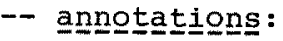

5.0 Interpreting a named statement list is the same as: 
5.3 interpreting that list as from its 1 st statement.

That is: the tixe (exit spelled in polish) clause of lines 5.1-5.2 is not 'executed' by the meta-language elaborator when first entering the int-Named-Stmt-list functions. We shall subsequently 'discover' the purpose of the tixe clause.

6.0 Having cued this function with $i$, i.e. having given it the startword $i$, interpretation of the statements of the (named) statement list

6.1 proceeds linearly, as from the cued, $i$ th, statement until the last -- provided, of course, meta-elaboration is not re-directed. When \& how this occurs will presently be uncovered.

\subsection{If the interpreted statement is}

7.2 a Goto, then an exit is performed. It is given an argument. This argument is the $L A B e l$ denotation, $m k-L A B(a i d, Z b l)$, by which this label is known in the environment. Meta-elaboration of an exit dynamically 'unravels' the meta-language block invocations. That is: we retrace our steps, back to (here) the most recent meta-language block having a tixe clause. We say that the tixe clause stops the exit.

5.1 The tixe clause we backtrack to, is the one associated with the named statement list of which the 'offending' Goto was an immediate statement.

The tixe clause is to be understood as follows: if the argument passed back with the exit $(7.2)$ is equal to some $m k-L A B(a i d, \eta)$ of the "map" component of the tixe clause, then the corresponding cue-int-Named-Stmt-list, with the appropriate cue position (i), is invoked; otherwise the despatching exit is not stopped here, but passed out to the next embracing tixe clause -- i.e. the Goto is to an embracing Brock.

6-7 In now (re-)interpreting the (same) named statement iist, but, possibly, as from another, cued, position, new Gotos may occur.

5.1 These are stopped, thus recursively, by the 'same' tixe clause. 
7.1 If the interpreted statement is instead, in contrast to annotations 7.1-7.3 above,

7.3 a Block, then that, nested, Block is interpreted.

And so on. exits are always stopped by an always clause before being passed on $(4.5-4.8)$.

\section{Comments}

The "maps" of tixe clauses may, as we have done in the above annotations, be considered not to be "computed" when the meta-language elaborator enters a meta-language block having a tixe clause. The domains of such "maps" are constrained to be statically determinable, and finite. Since, in the cue-int-... elaboration function, nst 2 is a static (i.e. fixed) object, this constraint is satisfied.

You may in fact view the domain of the "map" of the tixe clause as being 'computed' upon entry to the meta-language block of which it is part. Now, for this source language, the test for whether the "returned" argument of an exit belongs to such a "map" is likewise 'statically' decidable since only Gotos with constant Labet designators were permitted.

\subsection{The Exit Mechanisms}

In the following the language constructs, dealt with and mentioned, are those of the meta-language, and not constructs of a source language being modeled.

Variables presuppose declarations, and declarations define a state. Statements are requests for state changes, and denote state transformers, i.e. functions from states to states. The serial statement composition operator ";" (semicolon) thus denotes functional composition. In compound expressions all component sub-expressions are (usually) completely evaluated before any result value is yielded.

In this section we shall describe the only statement- and expression construct available for changing the meta-language statement interpre- 
tation-, respectively controlling the expression evaluation-, part.

The exit mechanism now to be explained offers -- in the context of statements -- in one sense a restricted form of, or alternative to conventional gotos. In particular: instead of providing, in the metalanguage, gotos to (arbitrary or phrase-structure constrained) labelled statements exits provide such gotos to statement-block or block-expression "ends".

In the context of an applicative language it is an altogether new construct.

The meta-language provides two kinds of exit designators:

$1,2 \quad$ exit, exit $(e x p r)$

and three kinds of exit stopping constructs:

$3.0 \quad \frac{\text { (always }}{\text { in }} \mathrm{F}(\ldots)$

4.0 (trap exit (def) with $F($ def)

$.1 \quad$ in $c(\ldots))$

$5.0 \quad$ (tixe $[G($ def $) \rightarrow F($ def $) \quad \mid P($ def $)]$

$.1 \quad$ in $c(\ldots))$

Lines $3.0,4.0$ and 5.0 schematize the stopping clauses. Any clause, $c(\ldots)$, prefixed by a stopping construct becomes a block.

Clauses 1 or 2 can occur where meta-language statements or expressions may occur. Thus the exit mechanism is an imperative, as well as an applicative construct -- see [Bjфrner 77e]. The use of the alwaye stopping clause, as will be seen, is, however, restricted to imperative blocks.

The forms def can be of either of the forms:

$$
\begin{aligned}
& \left\{d_{1}, d_{2}, \ldots, d_{n}\right\} \\
& \left.<d_{1}, d_{2}, \ldots, d_{n}\right\rangle \\
& m k-D\left(d_{1}, d_{2}, \ldots, d_{n}\right),\left(d_{1}, d_{2}, \ldots, d_{n}\right), \\
& i d_{2}, \text { or } \\
& \text { cst }
\end{aligned}
$$


where $i d$ stand for (free or bound) identifiers, est for constants (Iiterals), $d_{i}$ for forms of the above kind, and where the def form need not contain any free identifiers.

\subsection{Pragmatics \& Semantics of the Exit Mechanism}

The exit concept is based on the following four principles:

(I) The first basic principle of exit is to permit goto-like transfer of elaboration (statement interpretation, respectively expression evaluation) control to block boundaries -- i.e. to just outside their terminating part.

In particular: elaboration of any exit not definitively stopped in a block properly contained in $(.$.$) will result in immediate$ termination of any further parts of $c(\ldots)$, this to be followed by elaboration of some $F(\ldots)$.

(II) The second basic principle of exit is to permit the user to (implicitly) specify which (dynamically enclosing) block end the exits go to:

A block with no stopping clause is said to not definitively stop any exit. A trap exit unit whose elaboration may result in an exit is likewise said to not definitively stop an arbitrary exit. Finally: if an elaboration of $F(. .$.$) is completed with no exit then$ the exit is said to be definitively trapped. In that case elaboration of the block consists of elaboration of the part of $\mathcal{C}(\ldots)$ up to the exit followed by elaboration of the $F(\ldots)$. The potential state transformation yielded by an imperative block is in this case the serial composition of the two net effects. For the case the block is a block-expression $F(. .$.$) must in this case, namely that$ of definitive entrapment, yield a value.

(III) The third basic principle of exit is to permit the meta-language programmer to specify that certain actions be taken at any stopping block end.

The stop clauses serves this purpose. exits from multiply nested blocks may cause successive stopping actions, one per block (insideout), and each being terminated by an exit to the next enclosing block. 
An exit not definitively stopped by a stop clause of the (outermost) block of a function definition is dynamically passed to the immediately embracing block in which the actual reference to the function, i.e. "to this function definition", occurred.

The always stop clause unconditionally filters any exit, its $F(. .$.$) is elaborated, and the exit passed on to outer blocks.$ In consequence: exits of one function definition may, depending on dynamic calling patterns, be trapped by a multitude of stop clauses of blocks contained in various (other) function definitions. An exit of an activation of a recursively defined function may thus be stopped by a prior, temporarily suspended activation of that "same" function.

(IV) The fourth basic principle of the exit mechanism, i.e. the joint use of exits and stopping clauses is finally to communicate information from the (usually only dynamically determinable) point of exit to trap exit and tixe stop clauses' $F(\ldots)$. The idea being to let the elaboration of $F(. .$.$) depend on exit "returned" data.$

In particular: the value, $v$, of the expr of exit (expr) is obtained; the proper stop clause is found; and $v$ is substituted for all free occurrences of that unit's formal parameter def in that unit's $F(\ldots)$ resulting in $F^{\prime}(\ldots)$. Then $F^{\prime}(\ldots)$ is elaborated.

\section{Scope Rules}

An alternative way of describing some of the linguistic properties (of the exit mechanism) is now presented.

Two scope aspects are important: A syntactic (or static), and a semantic (or dynamic).

The syntactic scope rule is concerned with the scope of identifiers occurring in the def form of the trap exit clause. Identifiers of def, free in the embracing context, bind free occurrences of these identifiers in the corresponding $F(d e f)$. The static scope rules of the tixe "map" are the same as those of any implicit map (set or tuple) construction.

The semantic scope rule is concerned with the scope of the always, trap exit and tixe clauses. 
The dynamic scope rules of the always and trap exit clauses is the text $C(\ldots)$; whereas the dynamic scope rule of the tixe clause includes both the tixe "map" and the text $c(\ldots)$ !

Thus: an exit 'occurring' as a result of elaborating $F(d e f)$, of 3.0 or 4.0 , if not stopped in $F(\ldots)$, is not stopped by this $(3.0$, respectively 4.0) instance of the always, respectively trap exit clause. Instead it is passed out to possibly embracing meta-blocks.

An exit 'occurring' as a result of elaborating some $F(d e f)$ of 5.0 , i.e. of a tixe "map", if not trapped in $F(\ldots)$, is trapped by this $\underline{\text { tix }}$ ․ㅡ clause $(5.0)$. Thus the tixe clause is said to apply recurs

\section{Some Equivalence Transformations}

The always stop clause in:

$$
\frac{(\text { always } F(\ldots)}{\text { in } \mathrm{c}(\ldots))}
$$

is semantically equivalent to:

$$
\begin{aligned}
& \text { (trap exit(id) with }(F(\ldots) ; \text { exit(id)) } \\
& \text { in }(\ldots))
\end{aligned}
$$

In general a block with no stopping clause:

$$
\frac{(\operatorname{Let} x: E(\ldots) ;}{\mathcal{C}(\ldots))}
$$

is identical to a block with the simple gate:

$$
\begin{aligned}
& \text { (trap exit (id) with exit (id) } \\
& \text { in ( let : } E(\ldots) \text {; } \\
& \text { (...)) } \text {. }
\end{aligned}
$$

When no block termination actions are needed in an imperative block, then we write: 
Correspondingly, when the value passed back (by the exit) unconditionally is to become the result of the block, we write:

$$
\frac{(\operatorname{trap} e x i t}{\text { in }(i d) \text { with }} \text { id }
$$

or:

$$
\frac{(\text { trap }}{\text { in } c(\ldots i t)}(i d) \text { with return (id) }
$$

dependent on whether the block (-expression) is pure or impure.

Finally: 'mixed', nested uses of exit $(e)$ and exit, and thus e.g.

(trap exit with $V(\ldots)$ in $C(\ldots)$ ) and (trap exit(id) with $F(i d)$ in $\mathcal{C}(\ldots))$

do not make sense:

$$
\begin{aligned}
& \text { (trap exit (id) with } F_{j}(i d) \\
& \text { in (...) } \\
& \text { (trap exit with } F_{2}(\ldots) \\
& \text { in }(. . . \\
& \text { exit (expr) } \\
& \text {...1) } \\
& \text {...1) }
\end{aligned}
$$

Etcetera:

\section{Example 63:}

Continuing our directory example, from examples $19 \& 29$, we recall:

$$
\begin{aligned}
& D I R=\text { Rid } \vec{m} \text { RES } \\
& R E S=V A L \mid \text { DIR }
\end{aligned}
$$

with:

$$
\begin{aligned}
& \text { retrieve-res }(d i r, r i d l)= \\
& ((x i d l=<>) \rightarrow d i p, \\
& \text { T } \\
& \rightarrow \text { ( Let } \text { rid }=\underline{h a} \text { ridr } \text { in } \\
& \text { if } r i d \in \text { dom } d i r \\
& \text { then retrieve-res (dir (rid), til ridi) } \\
& \text { else undefined) }
\end{aligned}
$$

type: DIR Rid* $\stackrel{\sim}{\rightarrow} R E S$ 
Users of the system directory each have their qualified name, uid in $R i d^{*}$, otherwise guaranteed to designate a $D I R$ object:

$$
i s-D I R(r e t r i e v e-r e s(D i r, \text { uia) })
$$

Users issue resource names, $v i d$ in $R i d^{t}$, designating values in the glo= bal directory, $D i r$, as follows: let $d i r$ be the directory designated by $u i d$ :

$$
\text { Let } d i r=\text { retrieve-res }(D i r, \text { uid })
$$

If $v i d$ is some $\langle r i d\rangle$ then either rid names an entry in $d i r^{2}$, and we are through, the result is $d i r(x i d)$. Or rid does not name an entry in $d i r$. We now chop off the last Rid element of uid-- to resume the search as from the directory designated by the resulting ('remaining') uid". If $v i d$ is some $\left\langle m i d_{1}, x i d_{2}, \ldots, n i d_{n}\right\rangle$ for $n>1$, then $r i d_{1}$ either names a $D I R$ entry in $d i r$, and we search as from the designated directory, with resource name: $\left\langle m i d_{2}, \ldots, r i d_{n}\right\rangle$, or $r i d_{1}$ does not name a $D I R$ entry, and we 'back' up the directory hieraxchy, to a level one higher, i.e, nearer the root, than that at which we originally started, or at which the search which just failed took place. We complete the above incomplete description by giving the formal definition of the proper search algo= rithm:

search (uid, vid) (updown) (Dir) $=$

.1

(

.2

(trap exit with

.3

.4

if updown $=$ DOWN

.5

.6

.7

.8

.9

.10

.11

then exit

else if $u i d=<$

then undefined

else search(fst(uid), vid)(Uㄹ) (Dir) in

if Zen vid $=1$

then if hd vid $\in$ dom dir

then $d i x$ (had via)

else exit

.12

.13

.14

else if havid iom dir

then exit

else search(uidrahd vid>, tz vid) (DOWN) (Dir)))

type: $R i d^{*} R i a^{+} \sim((U P \mid D O W N) \sim(D I R \stackrel{R}{\rightarrow}(R E S))$ 
where:

$$
f s t(x i d l)=\langle\operatorname{rid}[i]| 1 \leq i \leq(\text { Len } x i d l)-1\rangle
$$

Given the global directory, Dir, which we could omit as a parameter, and a 'relative' resource name, vid, we initially invoke the above search function with the users identification and the updown marker set to $\underline{\underline{u}}$.

We may tie up any loose ends in your understanding of the search algo= rithm, by the following, alternative characterization: uid designates a node, $N$, in the directory hierarchy (or: "tree", see example 19). There are now three possibilities for vid. Either there is a downward path, towards the leaves, from $N$ whose sequence of edge labels is vid. Then vid designates the object 'hanging' on to the other end of this path (as seen from $N$ ). If there is no such path, starting at $N$, then vid might still designate an object in the hierarchy, but now as from a node $N^{\prime}$, between the root of the overall directory and $N$. Search starts with the node $N^{\prime}$ immediately above $N$.

The purpose of the updown marker is to guide the t프르 mechanism in backing up beyond already searched 'subtrees'.

The third possibility, for $v i d$, is that there is no resource, relative to $u i d$, that is named by it.

\section{Example 64 :}

The Zahn Event Mechanism [Zahn 74, Knuth 74, Halas 75, Bjørner 77 d] sche= matically looks like:

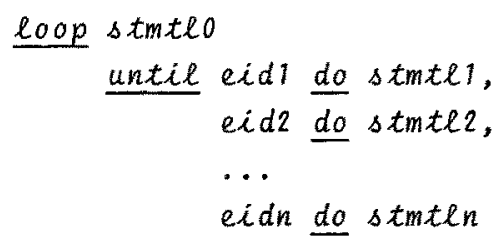

pool

An abstract syntax is: 
Zahn $:: \operatorname{Stmt}^{+}\left(E i d \vec{m}\right.$ Stmt $\left.^{+}\right)$

No statement in any stmtl $i \quad(1 \leq i \leq n)$ may name any event:

\section{event eid}

on any $\mathrm{eid}_{j}(1 \leq j \leq n)$. With:

Event $: \therefore$ Eid

as a statement, i.e. with:

Stmt $=$ Event $\mid$ Zahn $1 \ldots$

the static well-formedness criteria can be completely specified:

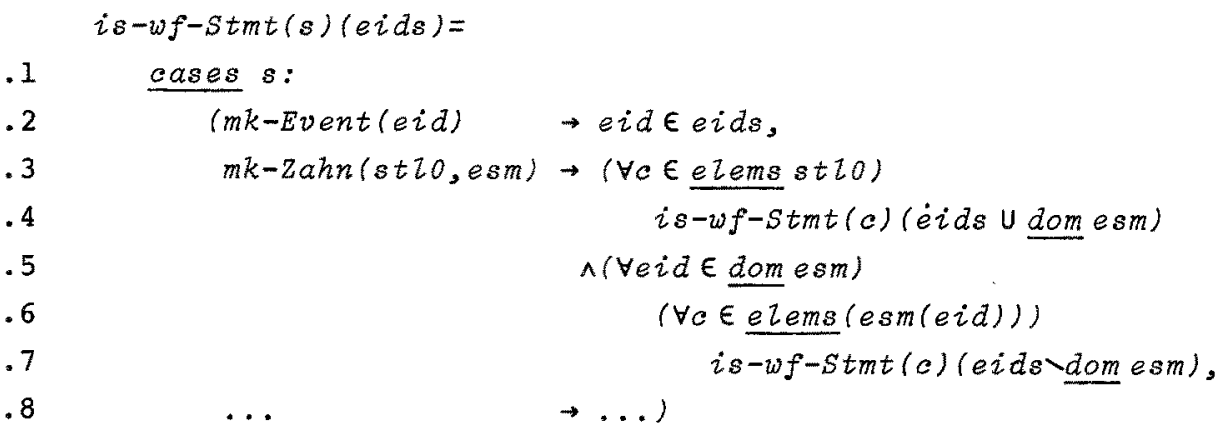

The informal, incomplete semantics is: stmtlo is repeatedly interpreted. If an event is interpreted in stmtlo then interpretation of stmtlo is terminated. If the event names some $e i d_{k}(1 \leq k \leq n)$, then the corresponding $\Delta t m t l_{k}$ is interpreted. This terminates interpretation of the mechanism. If the event names some properly containing mechanisms' $e i d_{\ell}$, then its stmtl $\ell_{\ell}$ is obeyed, ..., etc. Formally, we can capture things much more succinctly:

Assuming :

$$
\Sigma=(\underbrace{S T S T G) \cup(\ldots \ldots)}_{S T G}
$$

we get an imperative formulation:

type: int-Stmt: Stmt $\underset{\rightarrow}{\rightarrow}(E N V \cong(\Sigma \simeq \Sigma))$ 


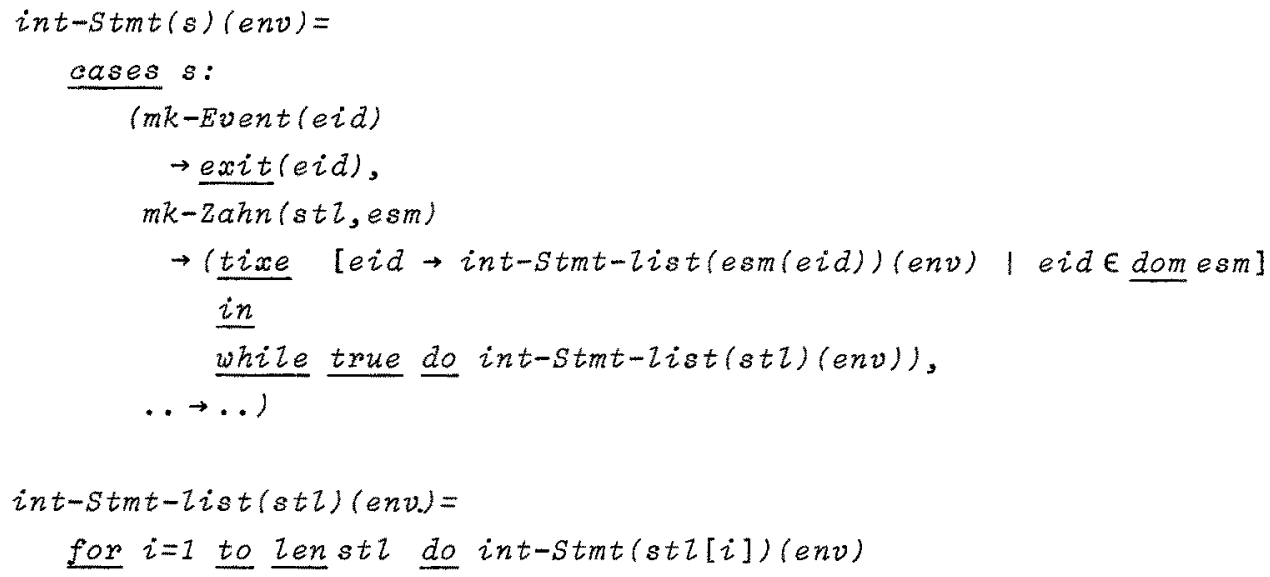

It is thus we observe that the tixe construct closely parallels the zahn event mechanism. The reader is,however, well-adviced to study [Jones $78 \mathrm{a}, 78 \mathrm{~b}$ ] in order to discover the neatness of the $\lambda$-calculus semantics given there for this meta-language combinator. 


\section{PART IV ABSTRACT MODELS}

Part I exemplified a complete, abstract model. Its components were: (I) abstract syntaxes for both syntactic \& semantic domains; (2) [static and dynamic] consistency constraints on objects of these domains -- called is-wf-... functions; (3) semantic elaboration-, and (4) auxiliary function definitions.

In part II, sections $2.1,3.1,4.1,5.1,6.1$ and 7 taught the notation for, and techniques of, defining domains. Sections $2.2,3.2,4.2,5.2$ and 6.2 taught the notation for representing objects of these domains. Sections $2.3,3.3,4.3,5.3$ and 6.3 finally taught the notation for, and techniques of, expressing transformations on objects.

Part III generally taught the notation for, and techniques of, stating processes, respectively composite transformations, on objects.

Part IV finally closes the story on the meta-language. With the bits and pieces of the meta-language introduced formally, and also heavily exemplified in parts II \& III, we can now formally introduce the notion of function definitions. Function definitions have, however, already been extensively exemplified. Section 12 will therefore wrap-up our tutorial on the meta-language. The section can best be understood if you frequently compare the formal information and schematized "examples" with examples 0-63: That is: we do not present comprehensive, abstract models involving function definitions, although those are its prime subjects: 


\section{Function Definitions \& Abstract Models}

In section 5 we introduced the notation for denoting domains of Function objects, for representing EunCTion objects, and for operations on Function objects. In a sense we are here continuing the story we started giving there.

\section{Example}

Recall the abstract models of e.g. examples $0,19,29,42,51-52,54-55,58-$ 59-60,61. On one hand we gave abstract syntaxes for syntactic domains, on the other we gave abstract syntaxes for semantic domains. The meaning of a syntactic object was a semantic object. The meaning of a Procedure, i.e. an object in Pre (example 40), is an object in $A R G^{*} \cong(\Sigma \sim \Sigma(\Sigma)$. The "thing" which takes a pro object and yields its denotation, i.e. an $A R G^{*} \stackrel{\sim}{\rightarrow}(\Sigma \tilde{\rightarrow} \Sigma)$ object, we call a semantic elaboration function (V-Pro). It is a function. This function, like all the FunCTion objects of section 5 , is described by the meta-language construct we call a function definition.

\subsection{The Syntax of Function Definitions}

A function definition consists of three parts:
a header:
$f i d\left(d_{1}, d_{2}, \ldots, d_{k}\right)\left(d_{k+1}\right) \ldots\left(d_{n}\right)=$
a body:
$c(\ldots)$
a type clause: type: $D_{1} D_{2} \ldots D_{k} \rightarrow\left(D_{k+1} \rightarrow\left(\ldots \rightarrow\left(D_{n} \rightarrow D^{\prime}\right) \ldots\right)\right.$

The body is any statement or expression clause you choose. The $d_{i}$ for $1 \leq i \leq n$ are usually formal parameter identifiers. They, or any identifiers occurring in $d_{i} ' s$, and the function name, $f i d$, bind free occurrences of these identifiers in $C(\ldots)$. The $D_{i}^{\prime} s$ and $D$ are domain expressions.

Observe the following relations between the $d_{i}$ 's of the formal parameter list and the $D_{i}$ 's of the type clause.

(1) If $d_{i}$ is of the form $\left\langle d_{i 1}, d_{i 2}, \ldots, d_{i m}\right\rangle$, the $D_{i}$ is either an identifier, and is then either the name of a domain defined by some abstract syntax rule: 


$$
\begin{aligned}
& D_{i}=D^{*}, \quad D_{i}=D^{*} \text { or } \\
& D_{i} \subset D^{*} ; \\
& \text { or } D_{i} \text { is (directly) either } D^{*} \text { or } D^{+} \text {- for some } D .
\end{aligned}
$$

(2) If $d_{i}$ is of the form $\left\{a_{i 1}, d_{i 2}, \ldots, a_{i m}\right\}$ then:

$$
D_{i}=D-s e t
$$

etcetera.

(3) If $d_{i}$ is of the form $m k-D_{i}\left(a_{i 1}, a_{i 2}, \ldots, a_{i m}\right)$, then there is an abstract syntax rule:

$$
D_{i} \quad \therefore D_{i 1} D_{i 2} \cdots D_{i m}
$$

(4) If $d_{i}$ is of the form $\left(d_{i 1}, d_{i 2}, \ldots, d_{i m}\right)$, respectively $m k\left(d_{i 2}, d_{i 2}, \ldots, d_{i m}\right)$, then $D_{i}$ is of the form:

$$
\left(D_{i 1} D_{i 2} \cdots D_{i m}\right)
$$

We do not presently see a need for writing $d_{i}^{\prime} s$ in forms other than the above, where ultimately $d_{i j}$ 's end up as identifiers, or letting the $d_{i}$ 's be identifiers. In the latter case $D_{i}$ 's may be any appropriate domain expression, not just an identifier. Experience shows forms $(1)-(2)$ to rarely occur.

\section{Example $6 \underline{5}:$}

This continues example 40 . Now, let:

$$
\begin{aligned}
& P R O C=A R G^{*} \cong(\Sigma \sim \Sigma) \\
& \text { PrC } \therefore I d^{*} \text { BZock }
\end{aligned}
$$

and:

$$
\begin{aligned}
& E N V=I d \vec{m} D E N \\
& D E N=L O C \mid \text { RROC }
\end{aligned}
$$

With these prerequisites we could also write the V-Pro elaboration function definition in this way: 


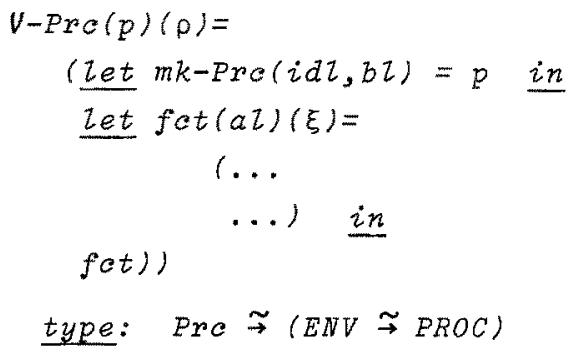

or

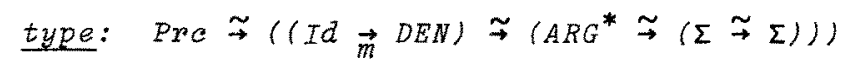

Relating the last type clause to the formal parameter list $((p)(p))$ we observe that $p$ is of type Pre, $\rho$ of type $\left(I d_{m} D E N\right)$, and the result yielded by $V$-Pro is whatever remains to the right of the arrow after the ENV specification:

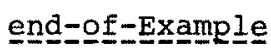

The form of $D$ remains to be constrained.

(5) If the body, $c(\ldots)$, of the function definition is a statement (-block), then $D$ is of the form:

$$
\Sigma \rightarrow \Sigma \text {, or: } \Sigma \simeq \Sigma
$$

where $\Sigma$ is the state resulting from global variable declarations.

(6) If the body, $C(\ldots)$, is instead an impure expression (-block), then $D$ is of the form:

$$
\Sigma \simeq\left(\Sigma D^{\prime}\right) \text {, or: } \Sigma \simeq\left(\Sigma D^{\prime}\right)
$$

where $D^{\prime}$ denotes the domain of results yielded by $\mathcal{C}(\ldots)$ with $\Sigma$ as in (5).

(7) If finally $\mathcal{C}(\ldots)$ is a pure expression, $D$ is any domain expression (etcetera -- concerning relation to $c(.$.$) ).$

\section{Example 6무:}

Observe that $D$ of e.g. the pure (applicative) I-Block, I-Stmt-list, and I-Stmt, definitions of example 42 , were all $\Sigma$. But then the $\Sigma$ was not 
that of any global variable state space -- since there was none --, but explicitly defined in example 42 formula 2 !

\section{end-of-example}

\section{Schönfinckeling/Currying}

The "back-to-back" ("dos-a-dos") parentheses, ")(", of the formal parameter list, e.g. to the left of $d_{i}$, may be replaced, starting with $i=k+1$, by commas "," -- provided we simultaneoursly delete the " $\rightarrow$ (" in the type clause, to the left of the corresponding $D_{i}$, and delete a (matching) right parentheses (")").

\section{Forrmal Exampleㅗㅡㅁ}

$$
f(a)(b)(c)(d)=C(\ldots) \text { type: } A \rightarrow(B \rightarrow(C \rightarrow(D \rightarrow E)))
$$

is one way,

$$
f(a, b)(c)(d)=\mathcal{C}(\ldots) \text { type: } A B \rightarrow(C \rightarrow(D \rightarrow E))
$$

is another way,

$$
f(a, b, c)(d)=C(\ldots) \text { type: } A B C \rightarrow(D \rightarrow E)
$$

and:

$$
f(a, b, c, d)=C(\ldots) \text { type: } A B C D \rightarrow E
$$

is a final way of following the so-called de-schönfinckeling or deCurrying rule given above.

The form:

$$
f^{\prime}(a)(b, c)(d)=c(\ldots) \text { type } A \rightarrow((B \quad C) \rightarrow(D \rightarrow E))
$$

is, however, not a currying of $f^{\prime}$ s definition above. De-Currying $f^{\prime}$ would lead to:

$$
f^{\prime}(a,(b, c), d)=c(\ldots) \text { type } A(B C) D \rightarrow E
$$

The conclusion to be drawn from the last example is that the type clause:

$$
\text { type: } g^{\prime}:\left(D_{1} D_{2} \cdots D_{n}\right)^{\prime} \rightarrow D
$$

is altogether distinct from:

$$
\text { type: } g^{\prime \prime}: D_{1} D_{2} \cdots D_{n} \rightarrow D
$$


Although we might (sloppily) write:

$$
g^{\prime}\left(d_{1}, d_{2}, \ldots, d_{n}\right)
$$

where, more correctly, we should write:

$$
g^{\prime}\left(\left(d_{1}, d_{2}, \ldots, d_{n}\right)\right)
$$

to distinguish it from the (correct):

$$
g^{\prime \prime}\left(d_{1}, d_{2}, \ldots, d_{n}\right)
$$

\section{end-of_-formal-examples}

The rule for removing ")("s etc. can be used in reverse -- and this was covered in section 6.2 (see subsection on $\lambda$-Expressions). In 6.2 we "Curry", whereas here we "de-Curry". As implied by the last, formal, example above, we must observe the dual role of parentheses: delimiting and anonymous tree domain \& tree object construction! If your keyboard has an extra pair of bracket/brace/parenthesis delimiters (other than our \{\}$, \longleftrightarrow,(),[]$, then use them to distinguish:

The Currying/de-Currying Rule is one form of syntactic transformation on function definitions. It involved the header/type-clause 'pair'. Semantics remained invariant!

\section{Formal Parameter Syntactic Transformations}

Another kind of syntactic transformation rule involves the header/body 'pair'. It can most simply be dealt with by listing the following semantic identities:

$$
\begin{aligned}
& \left.f\left(<d_{i 1}, d_{i 2}, \ldots, d_{i m}\right\rangle\right)=c(\ldots) \\
& = \\
& \left.f(d)=\left(\underline{\text { zet }}<d_{i 1}, d_{i 2}, \ldots, d_{i m}\right\rangle=d \underline{i n} c(\ldots)\right) \\
& f\left(m k-D_{i}\left(d_{i 1}, d_{i 2}, \ldots, d_{i m}\right)\right)=c(\ldots) \\
& \equiv \\
& f(d)=\left(\underline{\text { Let }} m k-D_{i}\left(d_{i 1}, d_{i 2}, \ldots, d_{i m}\right)=a_{\underline{i n}} c(\ldots)\right)
\end{aligned}
$$

etc.. It is here assumed that $d$ is not free in $c(\ldots)$. 


\subsection{The Semantics of Function Definitions \& Abstract Models}

\section{Scope \& Binding}

The identifiers occurring in the header of a function definition bind all their (free) occurrences in the body! Usually there is no need to re-define identifiers in any block.

\section{Gene토묘}

An abstract model consists of a set of tunction definitions, a set of abstract syntaxes, and possibly also a set of variable declarations. All names of defined functions, domains and variables are globally known.

The function denoted by any such, global function definition headers' first identifier, i.e. by the function identifier, fid, is the one you would expect. Formally: it is the least fix-point solution to the equation set that the function definitions form.

Informally we observe that an abstract model is all definitions -- and no real "action"! Nobody really invokes any of the specified functions.

An abstract model defines a class of systems.

\section{Examples}

Example 0 defined the structure and possible behavioral patterns of a class of grocery stores.

Examples $3-8,12-13,17-18,20-25,27-28$ \& 35 defined fragments of classes and manipulations of file systems.

Examples 19,29\&62 defined the structure and operations on a class of operating system directories.

Examples $30-34,36-38,40,42-43,47,49,61,63,70$ defined fxagments of structures and meaning of some class of programs, i.e. a source language.

If you come with a particular grocery store, or a particular file system, or a particular directory and a corresponding action (e.g. pur- 
chase, read, respectively catalog), then the definitions, i.e. the models, tell you what to expect happening. If you come with a particular state configuration and a program then the model will compute you a result.

However, you need not come with a semantic and a syntactic object. Come just with a syntactic object. And the model will produce the answer: this syntactic object denotes such-and-such a function from semantic objects to semantic objects.

If you are asking for the meaning of say a Purchase, example 0 , then "stick" your mk-Purchase(...) object into the Elab-Purchase semantic function. That is: you decide which part of your model to concentrate on. Therefore we, the model builders, do not tell you which one semantic function is the most important, $i$.e. which one should always be invoked first when "starting up" a model!

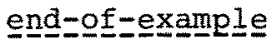

\section{A Note on Input/Output}

Based on the discussion (of example 69) it should now be easy to understand why the meta-language has no input loutput constructs.

A program in the meta-language defines a function. The meaning function from syntactic objects to semantic objects. The prime means for expressing this function is the set of function definitions. The abstract syn taxes describe the domain and range of this meaning function: the class of syntactic objects, and the class of semantic objects.

\section{PART V: MISCELLANEAE}

There remains only to formally introduce the elementary domains used in examples throughout this tutorial. 


\section{ELEMentary Data Types}

Usually a programming language comes ready-made, equipped with a set of basic data types.

A number of such have been employed in the examples given so far. These are:

.1 Rational NUMbers, INTeGers $(\ldots,-2,-1,0,1,2, \ldots)$, Natural numbers -- with the operations: $+,-, *,-,<, \leq,=, \neq, \geq,>$, exponentiation, ceiz, floor, etc.

.2 BOoLeans (true, false) -- with the operations: $\sim, v, \wedge, \supset$,.

.3 QUOTations $(\underline{A}, \underline{A} \underline{B}, \underline{A} \underline{B} \underline{C}, \ldots)$-- with the operations: =, $\neq$.

.4 TOKENS (-- for which no representation is required --) -- with the operations: $\#, \neq$.

Other elementary data types could be considered.

(A data type is a set of objects and a set of operations. For a data type to be elementary its objects must all be considered elementary, i.e. having no structure.

The data types so far formally introduced were:

SET; TUPLE; MAP, FCT; TREE

They were all CoMPosite. Thus the OBJects of the meta-language satisfy:

$$
\begin{aligned}
& \text { OBJ }=\text { ELEM | COMP } \\
& \text { COMP }=S E T \mid \text { TUPLE | MAP | FCT | TREE }
\end{aligned}
$$

where the five sub-domains of COMP are all considered disjoint.

In this tutorial we take ELEM to be:

$$
\text { ELEM = NUM | BOOL | QUOT | TOKEN }
$$

The following relations apply: 


$$
N_{1} \subset N_{0} \subset I N T G \subset N U M
$$

Otherwise the ELEM sub-domains are considered disjoint.)

If you need to introduce further objects or domains of objects -- by almost all means: At least as long as they are in keeping with the principles of abstractions (exemplified), and thus of the meta-language.

\subsection{Rational NUMbers}

Useful domains are:

NUM Rational numbers, i.e. the numbers that arise as the
result of integer (non-zero) divisions.
InTG
Integers
$N_{0} \quad \begin{aligned} & \text { Natural numbers, in particular the positive integers } \\ & \text { including } 0 .\end{aligned}$
$N_{1} \quad$ Natural numbers larger than or equal to 1.

We only find a need to represent the integers:

$$
\ldots,-3,-2,-1,0,1,2,3, \ldots
$$

Useful operators/operations are:

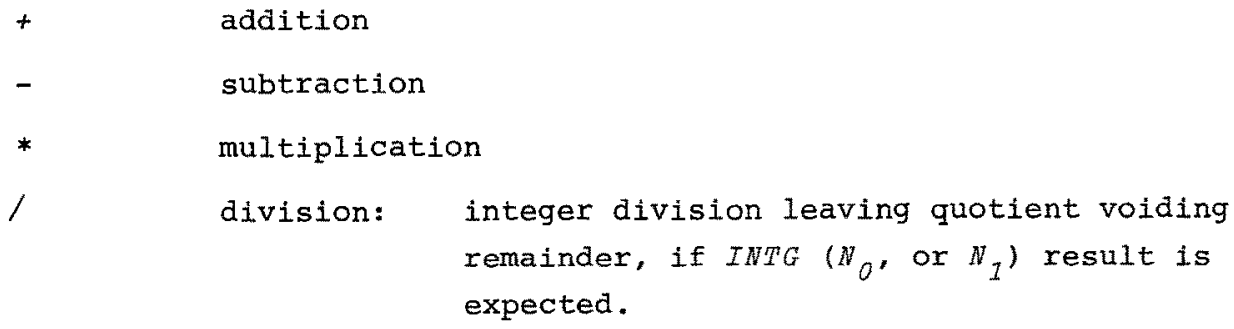

etc. If you would like to use:

ceit $r^{*}$ smallest integer larger than or equal to its only operand, floor largest integer smaller than or equal to its only operand, $i^{j} \quad$ the exponentiation of $i$ by $j$ 
etc., then we see no problem in you doing so. Etcetera -- for the introduction and proper explanation of your own operators (mod, $l_{n}$, rmd, sgn, ....).

\section{Programming Note}

We abstract numerals by the numbers they denote. Thus in example 49 constants of expressions are INTeGers (and BOoLeans).

\subsection{BOOLeans $\underline{\text { Logic Expressions }}$}

We name the domain in question:

BOOL Booleans values

In this tutorial we count on it having just two objects which we represent:

true, false

Useful operators/operations are:

$\begin{array}{ll}\sim & \text { negation, } \\ \vee & \text { or, } \\ \wedge, \& & \text { and (two forms provided), and } \\ \supset, \Rightarrow & \text { implication (two forms provided). }\end{array}$

In order to compact formulae we take the $\wedge, v,>$ operators as non-commutative! Thus if in $b 1 \wedge b a b 1$ is false, $b 2$ is never evaluated. Likewise if $b 1$ in $b 1 \mathrm{vb2}$ is true.

\section{Predicate Expressions}

Besides the propositional expressions which can be formed using expressions denoting booleans and the above, conventional operators, proper use of the meta-language heavily relies on the possibility of forming quantified expressions: 


$$
\begin{array}{ll}
(\forall x)(P(x)) & (\forall x \in \operatorname{set})(P(x)) \\
(\exists x)(P(x)) & (\exists x \in \operatorname{Set})(P(x)) \\
(\exists ! x)(P(x)) & (\exists ! x \in \operatorname{Set})(P(x))
\end{array}
$$

We 'read' the above expressions: "for all $x$ the predicate $P(x)$ is true", "for all $x$ in the set Set the predicate $P(x)$ is true", "there exists an $x$ for which the predicate $P(x)$ is true", "there exists an $x$ in the set Set for which the preaicate $P(x)$ is true", "there exists a unique $x$ for which $P(x)$ holds", and "there exists a unique $x$ in the set set for which $P(x)$ holds". In your reading the formulae 'aloud' you can e.g. vary as we did in the last two forms -- and you can instead of ' $x$ ' say 'objects $x^{\prime}$ or 'object $x^{\prime}$, etc.

\section{Examples}

We refer to examples $0,9,10,11,31$ (10), etc.

\section{Comments}

For the case where

$$
\text { set }=\left\{o_{1}, o_{2}, \ldots, o_{n}\right\}
$$

the quantified expressions can be simply transliterated:

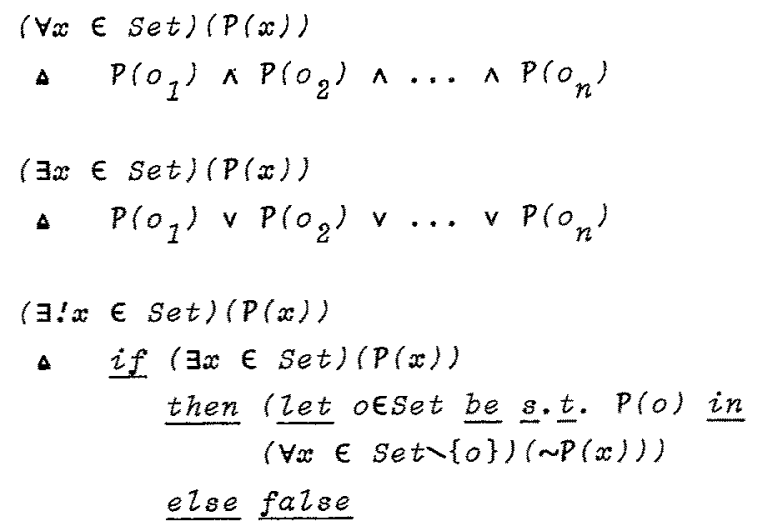




$$
\begin{array}{lll}
\sim(\exists x)(P(x)) & \equiv & (\forall x)(\sim P(x)) \\
\sim(\exists x \in S e t)(P(x)) & \equiv & (\forall x \in S e t)(\sim P(x))
\end{array}
$$

\subsection{QUOTations}

The subject domain is here named:

QUOT

Quotations are objects whose representations can be said to denote themselves. They are not to be confused with characters and characterstrings of conventional languages. We choose under-dashed sequences of (preferably) upper-case letters, and (rarely) other symbols:

$\underline{A}, \underline{B}, \ldots, \underline{Z}, \underline{A} \underline{A}, \underline{A} \underline{B}, \ldots, \underline{A} \underline{Z}, \underline{B} \underline{A}, \underline{B} \underline{B}, \ldots, \underline{B} \underline{Z}, \ldots, \underline{A} \underline{A} \cdots \underline{A}, \ldots$

The only two operations intended are:

$$
\begin{array}{ll}
= & \text { equality } \\
\neq & \text { non-equality }
\end{array}
$$

Q Quotations correspond to the enumerated type objects of PASCAL, but we define no ordering on them.) If you wish to use quotations to model characters then observe that $\underline{A} \underline{B C}$ is indivisible; and if you wish to use these modeling strings, then make tuples of quotations:

\section{Example 67:}

The following formulae may complete the syntax of example 49:

$$
\begin{aligned}
& \text { Int-OP = } \underline{A D} \underline{D}|\underline{S} \underline{U} \underline{B}| \underline{M P} \underline{\underline{Y}} \mid \underline{D} \underline{\underline{I}} \underline{V} \\
& B O O Z-O P=\underline{A N D}|\underline{O R}| \underline{I M P} \\
& R e Z-O p=\underline{L A R Q}|\underline{L A E Q}| \underline{E Q} \underline{\underline{Q}}|\underline{N E Q}| \underline{S M A E} \mid \underline{S M E Q}
\end{aligned}
$$

An evaluation function for the expressions of example 49 might use these quotations: 


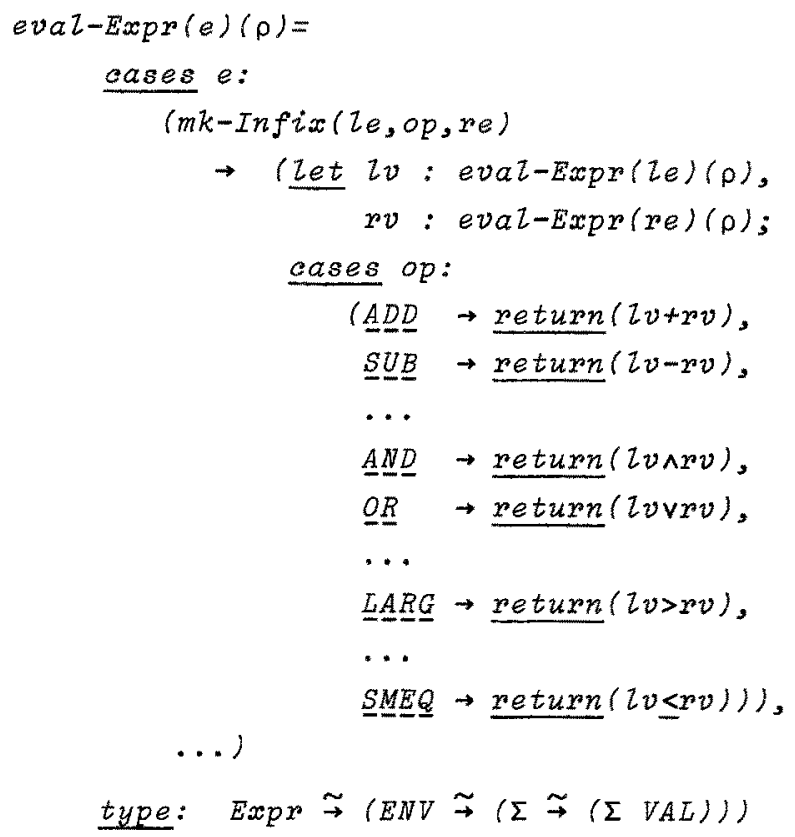

Here it was assumed that $l_{v}$ and $r v$ were of the appropriate type. Suppose the language is statically type checkable, then:

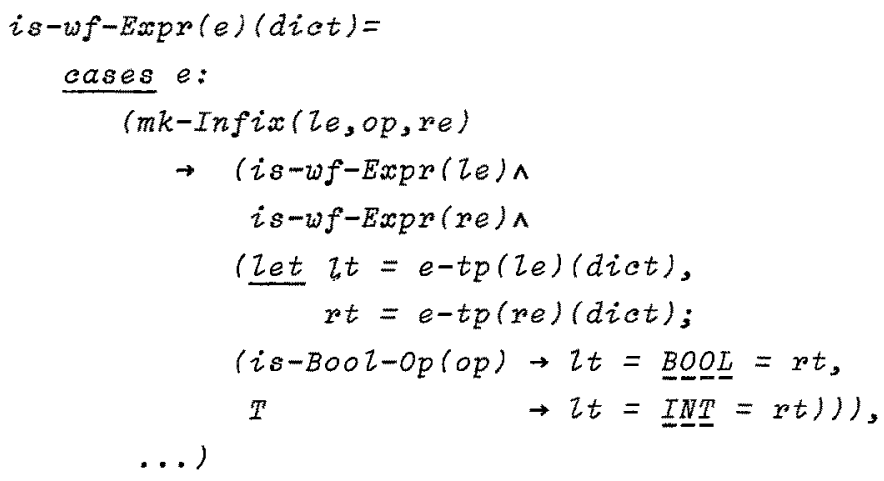

type: $\quad \operatorname{Exp} \rightarrow(D I C T \rightarrow B O O L)$

where:

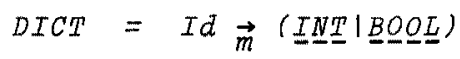

\subsection{POKENS}

The subject domain is named: 
TOKEN

Tokens are objects for which we seek no representation.

The only two operations provided are:

$$
\begin{array}{ll}
= & \text { equality } \\
\neq & \text { in-equality }
\end{array}
$$

Example 68 :

When modeling the identifiers of software systems (e.g. the operating system directory, examples $19 \& 29$; the file system file identifiers, examples $20,24-25,27-28 \& 36$; the source language variable procedure and formal parameter identifers, examples 30-31-32-33, 36 etcetera), when modeling labels of a goto language (example 61), or when modeling storage addresses, or as we call them: Locations (example 30-31) we use

\begin{tabular}{|c|c|c|}
\hline$R i d$ & $=$ TOKEN & (exs. 19), \\
\hline Fid & $=T O K E N$ & (exs. 20), \\
\hline Id & TOKEN & (exs, $30-33,36, \ldots)$, \\
\hline$L b z$ & $=T O K E N$ & $(\mathrm{ex}, 61)$ \\
\hline LOC & $=T O K E N$ & $(e x, 30)$, and \\
\hline ScaZar-Loc & $=T O K E N$ & (ex. 31). \\
\hline
\end{tabular}
TOKENS. That is:

When in e.g. examples 24-25 \& 27 we write suitably decorated fid's, these are meta-language identifiers naming otherwise unrepresented $F i d$ objects.

When in example 31 we need to 'generate' new Scalar-Locations, then we "pull them out of the scalar-Loe bag":

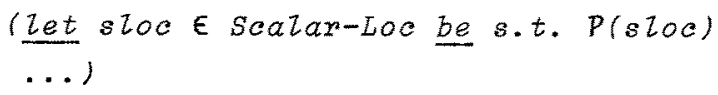


PART VI: POSTLUDE

This completes the informal introduction to the meta-language.

\section{ACKNOWLEDGEMENTS:}

The author is pleased to acknowledge all of his former colleagues of the IBM Vienna Laboratory, and Mrs.Jytte $\$ \varnothing l l e s t e d$ for her expert handling of eight IBM "golf ball" type-fonts: 


\section{EXAMPLE INDEX:}

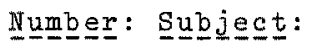

0. Grocery store.

1. School-Class of students.

2. Collections of classes of Integers.

3. Files: Unordered collec= tions of Records.

4. Collections of Integers.

5. Records, Files.

6.

7. File Manipulations \& Record Handling.

8. - - -

9. Equivalence classes.

10. Coarsest Partitionings.

11. Pascal Triangle.

12. Ordered Fields of Records. 13. - - -

\section{Meta-Language Constructets:}

A Complete Model: Abstract Syntax, Domains, Objects, Operations, Func= tion Definitions.

Section Introduction: sets, $\mathrm{Ab}=$ stract Syntax, Domains, Objects, Operations.

Set Domains.

Explicit Set Constructions.

Primitive Set Operations.

Imperative Set Operations.

Complete Specification: Abstract Syntax, Function Definitions.

Section Introduction: Tuples, Do= mains, Objects, Applicative \& Im= perative Function Definitions.

Tuple Domains, Abstract Syntax. Tuple Enumerations. 
14. Fibonacci Numbers

15. Pascal Triangle.

16. Value Stacks.

17. File Handling.

18. - -

19. System Directory.

20. File System \&iles.

21. Files.

22. - -

23. -"-

24. File System \& Files.

25. - - "

26. Conceptual Example.

27. File system \& Files.

28. -" -

29. System Directory.

30. Progr.Lang.:States \& Envi= ronments as Models of var= iables \& Scopes.
Tuple Enumerations.

Implicit Tuple Enumeration.

Tuple Domain \& Primitive Tuple operations.

Primitive Tuple Operations.

Applicative \& Imperative Tuple operations.

Section Introduction: Maps, $\mathrm{Ab}=$ stract Syntax, Domains, Objects, Operations, Function Definitions.

Map Domains.

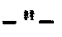

-"

$-1$

Explicit Map Constructions.

Implicit Map Constructions.

Implicit Map Construction: the scope of Identifiers, Abstract syntax.

Primitive Map Operations.

Imperative Map Operations.

Function Definitions, Map Opera= tions.

Map Domains, Primitive Operations, Abstract syntax. 
31. Prgr.Lang.: Model of Block Concept \& Storage.

32. Prgr.Lang.:Blocks.

33. $-"$

34. Prgr.Lang.: Statements.

35. System Directory \& Direc= tory update Commands.

36. Prgr. Lang.: Procedures.

37. Prgr.Lang.:Statements.

38.

39. Factorial Function.

40. Prgr. Lang.: Procedures.

41. Meta-Language: Type of Primitive Operations.

42. Prgr.Lang.:Blocks \& Sta= tements.

43. Prgr.Lang.

44-46. Conceptual Examples.

47. Prgr.Lang.: Syntax
Abstract Syntax, Map Domains, Function Definitions.

Trees, Syntactic Domains.

Trees, Abstract Syntax.

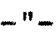

$-n$

$-"$

Selector Functions, Abstract syntax.

$-"-$

Section Introduction: Functions, Function Definitions, Block Ex= pressions.

Function Denotations, Semantic Domains, Syntactic Domains, $\mathrm{Ab}=$ stract Syntax, Elaboration Func= tions.

Function Types.

Abstract Syntax, Elaboration Function Definitions, Function Application.

Section Introduction: Abstract syntax.

Abstract Syntax.

is-functions. 
48. Reflexive Domains.

49. Prgr.Lang, :Expressions.

50. Factorial Function.

51.

Prgr.Lang.: Modeling Variables, Input, Output.

52. Prgr.Lang.:Assignment, Input, output.

53. Conceptual Example.

54. Prgr.Lang.:Structured statements.

55. Prgr. Lang . :For-To-Do statement.

56. Prgr.Lang .: Compound statement.

$57 . \quad-"-$

58. Prgr.Lang.:Arbitrary ordering.

59. -"-, For-Al1 statement.

60. Prgr.Lang.: While-Do
Abstract Syntax, Map Donains, Map Objects.

Abstract Syntax, McCarthy Condi= tional clause, Function Definition.

Section Introduction: Variables, Applicative \& Imperative Function Definitions, Expression Blocks, The state.

Global Variables, The State.

Elaboration- \& Auxiliary Function Definitions, Variables, Assign= ment.

Imperative \& Applicative Combi= nators.

Abstract Syntax, Semantic Domains, Structured clauses: cases, if. Applicative Definitions of Impe $=$ rative Features.

Abstract Syntax, Semantic Domains, Elaboration Function Definitions.

-"- \& for-to-do, Imperative De= finition.

-"-, but Applicative Definition.

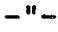

$-"$

Recursive ("sledge-hammer") Defi= nition of ("egg") while. 
61. Prgr.Lang.: Guarded Repe= titive Command.

62. Prgr.Lang.:GOTO \& Block Concept Model.

63. System Directory: Hierar= chical Entry Access.

64. Prgr.Lang.:Zahn's Event Mechanism.

65. Prgr.Lang,: Procedures.

66.

Prgr.Lang .: States.

67. Prgr.Lang. : Expressions.

68.
Prgr.Lang *: Names \& $\mathrm{Ad}=$ dresses.
Elaboration Function Definition, Applicative Definition.

$\underline{e x i t}$ and $\underline{\text { tixe }}$

$\underline{\text { exit }}$ and $\underline{t r a p}$

$\underline{\text { exit }}$ and $\underline{\text { tzxe }}$

Abstract Syntax, syntactic \& Se= mantic Domains, Elaboration Func= tion Definitions.

Imperative \& Applicative "States" .

Quotations.

Tokens. 